\title{
UVES analysis of red giants in the bulge globular cluster NGC 6522 ${ }^{\star}$
}

\author{
B. Barbuy ${ }^{1}$, E. Cantelli ${ }^{1}$, L. Muniz ${ }^{1}$, S. O. Souza ${ }^{1}$, C. Chiappini ${ }^{2}$, R. Hirschi ${ }^{3,4}$, G. Cescutti ${ }^{5,6}$, M. Pignatari ${ }^{7,8,9, \star \star, \star \star \star,}$ \\ S. Ortolani ${ }^{10,11}$, L. Kerber ${ }^{12}$, F. F. S. Maia ${ }^{13}$, E. Bica ${ }^{14}$, and E. Depagne ${ }^{15}$
}

${ }^{1}$ Universidade de São Paulo, IAG, Rua do Matão 1226, Cidade Universitária, São Paulo 05508-900, Brazil

e-mail: barbuy@astro.iag.usp.br

2 Astrophysikalisches Institut Potsdam, An der Sternwarte 16, Potsdam, 14482, Germany

3 Astrophysics Group, Keele University, Keele, Staffordshire ST5 5BG, UK

${ }^{4}$ Kavli Institute for the Physics and Mathematice of the Universe (WPI), University of Tokyo, 5-1-5 Kashiwanoha, Kashiwa 277-8583, Japan

${ }^{5}$ INAF, Osservatorio Astronomico di Trieste, Via G.B. Tiepolo 11, 34143 Trieste, Italy

${ }^{6}$ IFPU, Istitute for the Fundamental Physics of the Universe, Via Beirut, 2, 34151, Grignano, Trieste, Italy

${ }^{7}$ E.A. Milne Centre for Astrophysics, Department of Physics \& Mathematics, University of Hull, Hull HU6 7RX, UK

${ }^{8}$ Konkoly Observatory, Research Centre for Astronomy and Earth Sciences, Hungarian Academy of Sciences, Konkoly Thege Miklos ut 15-17, 1121 Budapest, Hungary

${ }^{9}$ Joint Institute for Nuclear Astrophysics - Center for the Evolution of the Elements, USA

${ }^{10}$ Università di Padova, Dipartimento di Astronomia, Vicolo dell'Osservatorio 2, 35122 Padova, Italy

11 INAF-Osservatorio Astronomico di Padova, Vicolo dell'Osservatorio 5, 35122 Padova, Italy

${ }^{12}$ Universidade Estadual de Santa Cruz, Depto. de Ciências Exatas e Tecnológicas, Rodovia Jorge Amado km 16, 45662-900, Ilhéus, Brazil

${ }^{13}$ Universidade Federal do Rio de Janeiro, Instituto de Física, Av. Athos da Silveira Ramos, 149, 21941-972, Rio de Janeiro, Brazil

${ }^{14}$ Universidade Federal do Rio Grande do Sul, Departamento de Astronomia, CP 15051, Porto Alegre 91501-970, Brazil

${ }^{15}$ South African Astronomical Observatory (SAAO), PO Box 9, Observatory 7935, South Africa

Received 15 March 2021 / Accepted 3 July 2021

\section{ABSTRACT}

Context. NGC 6522 is a moderately metal-poor bulge globular cluster $([\mathrm{Fe} / \mathrm{H}] \sim-1.0)$, and it is a well-studied representative among a number of moderately metal-poor blue horizontal branch clusters located in the bulge. The NGC 6522 abundance pattern can give hints on the earliest chemical enrichment in the central Galaxy.

Aims. The aim of this study is to derive abundances of the light elements $\mathrm{C}$ and $\mathrm{N}$; alpha elements $\mathrm{O}, \mathrm{Mg}, \mathrm{Si}, \mathrm{Ca}$, and $\mathrm{Ti}$; odd- $\mathrm{Z}$ elements $\mathrm{Na}$ and $\mathrm{Al}$; neutron-capture elements $\mathrm{Y}, \mathrm{Zr}, \mathrm{Ba}, \mathrm{La}$, and $\mathrm{Nd}$; and the $r$-process element Eu. We verify if there are first- and second-generation stars: we find clear evidence of $\mathrm{Na}-\mathrm{Al}, \mathrm{Na}-\mathrm{N}$, and $\mathrm{Mg}-\mathrm{Al}$ correlations, while we cannot identify the $\mathrm{Na}-\mathrm{O}$ anticorrelation from our data.

Methods. High-resolution spectra of six red giants in the bulge globular cluster NGC 6522 were obtained at the 8m VLT UT2-Kueyen telescope with both the UVES and GIRAFFE spectrographs in FLAMES+UVES configuration. In light of Gaia data, it turned out that two of them are non-members, but these were also analysed. Spectroscopic parameters were derived through the excitation and ionisation equilibrium of Fe I and Fe II lines from UVES spectra. The abundances were obtained with spectrum synthesis. Comparisons of abundances derived from UVES and GIRAFFE spectra were carried out.

Results. The present analysis combined with previous UVES results gives a mean radial velocity of $v_{\mathrm{r}}^{\text {hel }}=-15.62 \pm 7.7 \mathrm{~km} \mathrm{~s}^{-1}$ and a metallicity of $[\mathrm{Fe} / \mathrm{H}]=-1.05 \pm 0.20$ for NGC 6522 . Mean abundances of alpha elements for the present four member stars are enhanced with $[\mathrm{O} / \mathrm{Fe}]=+0.38,[\mathrm{Mg} / \mathrm{Fe}]=\approx+0.28,[\mathrm{Si} / \mathrm{Fe}] \approx+0.19$, and $[\mathrm{Ca} / \mathrm{Fe}] \approx+0.13$, together with the iron-peak element $[\mathrm{Ti} / \mathrm{Fe}] \approx+0.13$, and the $r$-process element $[\mathrm{Eu} / \mathrm{Fe}]=+0.40$. The neutron-capture elements $\mathrm{Y}, \mathrm{Zr}$, Ba, and La show enhancements in the $+0.08<[\mathrm{Y} / \mathrm{Fe}]<+0.90,0.11<[\mathrm{Zr} / \mathrm{Fe}]<+0.50,0.00<[\mathrm{Ba} / \mathrm{Fe}]<+0.63,0.00<[\mathrm{La} / \mathrm{Fe}]<+0.45$, and $-0.10<[\mathrm{Nd} / \mathrm{Fe}]<+0.70 \mathrm{ranges}$. We also discuss the spread in heavy-element abundances.

Key words. stars: abundances - globular clusters: individual: NGC 6522 - Galaxy: bulge

\section{Introduction}

The Galactic bulge formation was probably a result of early mergers and/or dissipative collapse combined with a buckling

\footnotetext{
* Observations collected at the European Southern Observatory, Paranal, Chile (ESO), under programmes 088.D-0398A, and 097.D0175 (PI: B. Barbuy), 071.B-0617, 73.B-0074 (PI: A. Renzini),

$\star \star$ WWW . nugridstars.org

$\star \star \star$ NuGrid Collaboration.
}

bar, as suggested by the excellent modern data now available in the innermost parts of the Galaxy (Queiróz et al. 2020a,b; Rojas-Arriagada et al. 2020; Pérez-Villegas et al. 2020; Kunder et al. 2020; Savino et al. 2020 - see also review by Barbuy et al. 2018a), and chemodynamical models (e.g. Fragkoudi et al. 2020; Debattista et al. 2020; Baba \& Kawata 2020). Within this context, globular clusters are important tracers of the early formation of the Galactic bulge, assuming that most of them were formed in situ. In particular, their abundance pattern could give hints as to the early nucleosynthesis processes in the Galaxy. 
The globular cluster NGC 6522 located in the Galactic bulge was identified by Baade (1946) as having a type II stellar population given its colour-magnitude diagram (CMD); hence, it falling into the Population II class defined in Baade (1944). Despite such an early identification of this cluster, it has not been widely studied since then.

NGC 6522 is an old globular cluster, with a moderate metallicity of $[\mathrm{Fe} / \mathrm{H}] \sim-1.0$, and a blue horizontal branch. Several other such clusters are present in the Galactic bulge, such as NGC 6558 (Rich et al. 1998; Barbuy et al. 2007, 2018b), HP 1 (Barbuy et al. 2006, 2016), AL 3 (Ortolani et al. 2006), Terzan 9 (Ernandes et al. 2019), and UKS 1 (Fernández-Trincado et al. 2020). Rossi et al. (2015) presented a study gathering these inner globular clusters, that might represent the earliest stellar populations in the Galaxy. Based on their derived proper motions, Rossi et al. (2015) concluded that the inner bulge globular clusters have clearly lower transverse motions and spatial velocities than halo clusters, and they appear to be trapped in the bulge bar.

Pérez-Villegas et al. (2020) computed the orbits of 78 inner Galaxy globular clusters, following the selections given in Bica et al. (2016). It was found that most of the confirmed bulgepopulation clusters are confined in the bar region but are not supporting the bar structure. For each cluster, a set of 1000 initial conditions were generated, following the Monte Carlo technique and taking into account the observational uncertainties. NGC 6522 has a $99.8 \%$ probability of being a bulge member, and most of the orbits among the different initial conditions do support the bar shape. A study of the origin of Galactic globular clusters by Massari et al. (2019) also identified NGC 6522 as having been formed in the main bulge and not, for example, in the Gaia-Enceladus that merged with the Galaxy about $10 \mathrm{Gyr}$ ago. At the same time, it was confirmed to be an old cluster by Kerber et al. (2018): 14.1, $14.2 \mathrm{Gyr}$ for $[\mathrm{Fe} / \mathrm{H}]=-1.0,-1.15$ from BaSTI isochrones (Pietrinferni et al. 2004, 2006) and 12.1, $12.4 \mathrm{Gyr}$ for $[\mathrm{Fe} / \mathrm{H}]=-1.0,-1.15$ from Dartmouth isochrones (Dotter et al. 2008). This old age indicates that NGC 6522 was formed $4 \mathrm{Gyr}$ prior relatively to the estimated age of the bar formation of $8 \pm 2$ Gyr by Buck et al. (2018) and $~ 8$ Gyr ago by Bovy et al. (2019). Therefore, the fact that NGC 6522 follows the bar probably indicates that it was confined within the bar when the latter formed.

Barbuy et al. (2009) analysed eight member stars based on FLAMES-GIRAFFE (Pasquini et al. 2002) spectra, included in the survey by Zoccali et al. (2008). Even with these lowresolution spectra (as compared with the UVES spectra analysed later) it was possible to detect some enrichment in $s$-process elements, which Chiappini et al. (2011a, hereafter C11) interpreted as a possible signature of an early generation of fast rotating stars (the so-called spinstars). This was based on the idea that, as the age-metallicity relation in the bulge was expected to be steeper than in the halo, it would already be possible to reach metallicities as large as $[\mathrm{Fe} / \mathrm{H}] \sim-1$ on a very short timescale. It was then suggested that in the bulge, globular clusters at $[\mathrm{Fe} / \mathrm{H}] \sim-1.0$ would already represent tracers of the earliest chemical enrichment phases. Subsequently, we obtained UVES spectra for four of the stars previously analysed in Barbuy et al. (2009) and C11. The re-analysis of these stars based on higher resolution and higher signal-to-noise $(\mathrm{S} / \mathrm{N})$ spectra, obtained with the UVES spectrograph at the Very Large Telescope was then presented in Barbuy et al. (2014) where some enrichment of $s$-process elements in the very old NGC 6522 cluster has been confirmed, although smaller than what was suggested in the earlier low-resolution spectra. It was then necessary to expand the sample to better constrain the nature of the stars that have polluted this very old cluster. With this aim, during our first UVES observations we also obtained parallel observations with the FLAMES-GIRAFFE spectrograph, and new candidate members were identified. These, in turn, were observed with UVES in a new run. In this work, we present results for six stars in NGC 6522 obtained in 2016 with the FLAMESUVES spectrograph (Dekker et al. 2000). Our main aim is to study the abundance signatures of heavy elements in the cluster.

Furthermore, NGC 6522 was recently shown by Kerber et al. (2018) to have at least two stellar populations in proportions of $86 \%$ as second generation $(2 \mathrm{G})$ and $14 \%$ as first generation $(1 \mathrm{G})$. For this reason, we inspected possible $\mathrm{Na}-\mathrm{O}$ anti-correlations and $\mathrm{Na}-\mathrm{Al}, \mathrm{Na}-\mathrm{N}$, and $\mathrm{Mg}-\mathrm{Al}$ correlations (Gratton et al. 2012 and references therein) among the present sample stars together with stars analysed in Barbuy et al. (2014).

Finally, we compare element abundance derivation from UVES and GIRAFFE spectra for stars observed with both instruments (in their common wavelength region) to check for further use of the lower resolution spectra. Additionally, in Table A.1 in the appendix we list stars identified to be candidate members of the cluster, observed with FLAMES-GIRAFFE, selected from their radial velocities together with Gaia Collaboration (2018, 2021) proper motions.

The observations are described in Sect. 2. Photometric stellar parameters' effective temperature and gravity are derived in Sect. 3. Spectroscopic parameters are derived in Sect. 4, and abundance ratios are computed in Sect. 5. A discussion is presented in Sect. 6, and conclusions are drawn in Sect. 7.

\section{Observations}

In Barbuy et al. (2009), eight stars of NGC 6522 observed with the GIRAFFE spectrograph, within the list of over 600 bulge stars analysed by Zoccali et al. (2008) (programs 71.B-0617 and 73.B-0074, PI: A. Renzini) were studied. Four of these were reobserved at higher resolution with UVES and analysed in Barbuy et al. (2014), in programme 88.D-0398 (PI: B. Barbuy) in 2012. From GIRAFFE stars observed in the same field within this same programme, we identified stars with radial velocities and metallicities that could be cluster members. In program 097.D0175 (PI: B. Barbuy), we observed five of these stars, plus star $\mathrm{B} 118$, which was previously studied at a moderate resolution in Barbuy et al. (2009). The log-book of observations is given in Table 1.

The UVES spectra were obtained using the FLAMES setup centred at $580 \mathrm{~nm}$, with a coverage ranging from $480 \mathrm{~nm}$ to $680 \mathrm{~nm}$. The 2012 GIRAFFE spectra were obtained using setups HR11 (559.7-584.0 nm) and HR12 (582.1-614.6 nm), and the 2016 GIRAFFE observations used setups HR11, HR13 (612.0$640.5 \mathrm{~nm})$, and HR14A $(630.8-670.1 \mathrm{~nm})$, all with a mean resolving power of $R \approx 22000$.

The UVES data were reduced with the UVES pipeline v5.7.0 within the REFLEX ambient. The extracted spectra were treated, normalised, rest-frame aligned, and combined using the method described in Sect. 2.3 of Cantelli (2019). Cosmic-ray removal was done by sigma rejection. Radial velocities were measured using the upper wavelength chip of the UVES red arm, through the IRAF task fxcor, using the Arcturus spectrum as template (Hinkle et al. 2000). The measurements for each star and each exposure and the heliocentric radial velocities computed through the IRAF task rvcorrect are reported in Table 2. 
Table 1. Log of the spectroscopic observations of programs 088.D0398(A) and 097.D-0175(A), carried out in 2011-2012 and 2016, respectively.

\begin{tabular}{|c|c|c|c|c|}
\hline Date & UT & $\begin{array}{c}\text { Julian } \\
\text { date }\end{array}$ & $\begin{array}{l}\text { Exp } \\
(\mathrm{s})\end{array}$ & $\begin{array}{l}\text { Air- Seeing } \\
\text { mass ('") }\end{array}$ \\
\hline \multicolumn{5}{|c|}{ Program 088.D-0398(A) } \\
\hline 2011-10-08 & $00: 45: 54$ & 2455842.53187 & 2750 & $1.4620 .82^{\prime \prime}$ \\
\hline 2011-10-08 & $01: 34: 37$ & 2455842.56571 & 2750 & $1.8531 .29^{\prime \prime}$ \\
\hline 2012-03-06 & $07: 38: 32$ & 2455992.81843 & 2750 & $1.5791 .15^{\prime \prime}$ \\
\hline 2012-03-06 & $08: 28: 44$ & 2455992.85329 & 2750 & $1.2600 .93^{\prime \prime}$ \\
\hline 2012-03-07 & $07: 47: 56$ & 2455993.82495 & 2750 & $1.4890 .81^{\prime \prime}$ \\
\hline 2012-03-07 & 08:39:16 & 2455993.86060 & 2750 & $1.2700 .73^{\prime \prime}$ \\
\hline 2012-03-25 & $08: 31: 47$ & 2456011.85541 & 2750 & $1.0870 .64^{\prime \prime}$ \\
\hline \multicolumn{5}{|c|}{ Program 097.D-0175(A) } \\
\hline 2016-05-17 & $07: 22: 18$ & 2457525.80716 & 2400 & $1.0070 .40^{\prime \prime}$ \\
\hline 2016-05-17 & 08:05:08 & 2457525.83690 & 2400 & $1.0330 .47^{\prime \prime}$ \\
\hline 2016-05-17 & $08: 52: 35$ & 2457525.86985 & 2400 & $1.0990 .47^{\prime \prime}$ \\
\hline 2016-07-11 & $02: 33: 35$ & 2457580.60666 & 2400 & $1.0280 .96^{\prime \prime}$ \\
\hline 2016-07-21 & $03: 27: 16$ & 2457590.64394 & 2400 & $1.0160 .51^{\prime \prime}$ \\
\hline 2016-07-21 & $04: 43: 37$ & 2457590.69696 & 2400 & $1.1120 .54^{\prime \prime}$ \\
\hline 2016-07-21 & $06: 33: 32$ & 2457590.75246 & 2400 & $1.3730 .54^{\prime \prime}$ \\
\hline 2016-07-22 & $04: 48: 26$ & 2457591.70031 & 2400 & $1.1310 .48^{\prime \prime}$ \\
\hline 2016-07-22 & $05: 40: 15$ & 2457591.73629 & 2400 & $1.2880 .45^{\prime \prime}$ \\
\hline 2016-07-22 & $06: 33: 29$ & 2457591.77326 & 2400 & $1.5740 .63^{\prime \prime}$ \\
\hline
\end{tabular}

Notes. The seeing and airmasses reported are the mean values along the exposures.

For completeness, and thanks to the Gaia Collaboration (2018, 2021) measurements, we were able to proceed to a more robust verification of membership. In Table 3, we list the proper motions $\left(\mu_{\alpha} \cos \delta\right.$ and $\left.\mu_{\delta}\right)$ and $G$ magnitude from Gaia Early Data Release 3 (EDR3, Gaia Collaboration 2021). With the precision improvement of Gaia EDR3 on proper motions, we recalculated the mean values for NGC 6522. We selected stars within 15 arcmin of the cluster centre and applied the Gaussian mixture models (GMM) to separate the cluster stars from field stars. With this method, we recalculated the cluster proper motion as

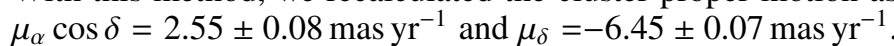
Also, the cluster and field distributions allowed us to determine the membership probability for each star. As shown in Table 3, and in the point-vector diagram of Fig. 1, stars 234816 and 244523 turn out to be non-members. This shows the power of Gaia, given that these two stars have the very compatible metallicities and radial velocities, coinciding with the other four member stars in the CMD. This is even more striking given that only $0.5 \%$ of field stars show a metallicity of $[\mathrm{Fe} / \mathrm{H}] \lesssim-1.0$ in the Galactic bulge (Barbuy et al. 2018a).

A mean heliocentric radial velocity of $v_{\mathrm{r}}^{\text {hel }}=-16.96 \mathrm{~km} \mathrm{~s}^{-1}$ is found for the four UVES sample member stars. A mean value of $v_{\mathrm{r}}^{\text {hel }}=-14.3 \pm 3.3 \mathrm{~km} \mathrm{~s}^{-1}$ was obtained from UVES spectra of four stars analysed in Barbuy et al. (2014). By combining the present data with these four stars, namely, B-107, B-128, B-122 and B-130, with $v_{\mathrm{r}}^{\text {hel }}=-7.626,-14.651,-18.043$, and $-16.808 \mathrm{~km} \mathrm{~s}^{-1}$, respectively, we obtain a mean value of $v_{\mathrm{r}}^{\text {hel }}=$ $-15.62 \mathrm{~km} \mathrm{~s}^{-1}$. A range of velocities between $v_{\mathrm{r}}^{\text {hel }}=-7.63$ and $-22.57 \mathrm{~km} \mathrm{~s}^{-1}$ gives a dispersion of $\pm 7.7 \mathrm{~km} \mathrm{~s}^{-1}$. A similar range of radial velocities was detected in Fernández-Trincado et al. (2019), including stars with $-21.97<v_{\mathrm{r}}^{\text {hel }}<-6.61 \mathrm{~km} \mathrm{~s}^{-1}$.

The GIRAFFE data were retrieved from the ESO reduced data archive. The extracted spectra belonging to the same setups were then corrected for radial velocity, normalised, and combined by the median. In the appendix, we give a list of new candidate member stars observed with GIRAFFE in 2012 and 2016 , within a radial velocity range of $-14.5 \pm 12 \mathrm{~km} \mathrm{~s}^{-1}$, that are confirmed members from proper motions.

\section{Photometric stellar parameters}

\subsection{Temperatures}

The selected stars, their OGLE and 2MASS designations, coordinates, and $V I J H K_{\mathrm{s}}$ magnitudes are given in Table $4 . V$ and $I$ data were collected from the Optical Gravitational Lensing Experiment (OGLE) survey, the OGLE-II release, Field BulSC45 centered at 18:03:33.0, -30:05:00 from Udalski et al. (2002). 2MASS $J, H$, and $K_{\mathrm{s}}$ are from Skrutskie et al. $(2006)^{1}$, and VVV $J, H$, and $K_{\mathrm{s}}$ magnitudes are from the Vista Variables in the Via Lactea survey (Saito et al. 2012) ${ }^{2}$. Table 3 reports the (Gaia Collaboration 2018, 2021) $G$ magnitudes and deduced $B$ magnitudes by applying the transformation $G_{\mathrm{mag}}-V_{\mathrm{C}}=f(B-$ $V)$ from Riello et al. (2021). In Fig. 2, we show the location in $B, V$ of the sample stars, in the CMD of NGC 6522 from data observed in $F 435 \mathrm{~W}$ and $F 555 \mathrm{~W}$ with the Hubble Space Telescope, and converted to $B$ and $V$ by Piotto et al. (2002).

Photometric effective temperatures and bolometric corrections were derived from $V-I, V-K$, and $J-K$ using the colour-temperature calibrations of Alonso et al. (1999, hereafter AAM99) and Casagrande et al. (2010, hereafter C10). For the transformation of $V-I$ from the Cousins to Johnson system, we used $(V-I)_{C}=0.778(V-I)_{J}$ (Bessell 1979). J, H, and $K_{\mathrm{S}}$ 2MASS magnitudes and colours were transformed from the 2MASS system to the California Institute of Technology (CIT), and from this to TCS (Telescopio Carlos Sánchez), following Carpenter (2001) and Alonso et al. (1998). The VVV $J H K_{s}$ colours were transformed to the 2MASS $J H K_{\mathrm{s}}$ system using relations by Soto et al. (2013) and then transformed to CIT as above.

The derived photometric effective temperatures, which are adopted as initial guesses, resulting from relations from AAM99 and $\mathrm{C} 10$ are both listed in Table 5. The differences in effective temperatures, in the $\Delta T_{\text {eff }}(\mathrm{C} 10-\mathrm{AAM} 99)$ sense, are of $+64.7 \mathrm{~K}$, $+54.2 \mathrm{~K}$ and $\pm 140 \mathrm{~K}$ for $V-I, V-K$ and $J-K$, respectively. These temperatures are used only as a guide to start fitting them from the $\mathrm{Fe} \mathrm{I}$ and $\mathrm{Fe}$ II lines.

\subsection{Gravities}

For a derivation of photometric gravities, we used the classical

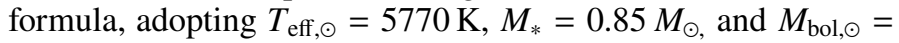
4.75. For the cluster, we used a distance modulus of $(m-M)_{0}=$ 14.40 and a reddening of $E(B-V)=0.52$ and $A_{\mathrm{V}}=1.61$, based on Kerber et al. (2018). Bolometric corrections were derived using AAM99 and $\mathrm{C} 10$ assuming $\mathrm{BC}_{V, \odot}=-0.07, M_{V, \odot}=4.81$, and $M_{I, \odot}=4.10$ from Willmer (2018). The computed bolometric magnitudes and gravities are given in Table 5.

\section{Spectroscopic stellar parameters}

The equivalent widths (EW) of Fe I and Fe II lines were measured using IRAF. The EWs, together with wavelength $(\AA)$; excitation potential $(\mathrm{eV})$, damping constant $\mathrm{C}_{6}$ and oscillator

\footnotetext{
1 https://irsa.ipac.caltech.edu/frontpage/;

https://irsa.iapc.caltech.edu

2 horus.roe.ac.uk/vsa
} 
Table 2. Observed and heliocentric radial velocities of the UVES sample stars, in each of the 10 UVES exposures, and the mean heliocentric radial velocity.

\begin{tabular}{|c|c|c|c|c|c|c|c|c|c|c|c|c|c|}
\hline \multirow{2}{*}{$\frac{\text { OGLE }^{\circ}}{\text { Date }}$} & \multirow{3}{*}{ UT } & \multicolumn{2}{|c|}{234816} & \multicolumn{2}{|c|}{244523} & \multicolumn{2}{|c|}{244819} & \multicolumn{2}{|c|}{256289} & \multicolumn{2}{|c|}{ B118 } & \multicolumn{2}{|c|}{402370} \\
\hline & & \multicolumn{12}{|c|}{ Observed and heliocentric radial velocity $\left(\mathrm{km} \mathrm{s}^{-1}\right)$} \\
\hline & & $v_{\text {obs }}$ & $v_{\text {hel }}$ & $v_{\mathrm{obs}}$ & $v_{\text {hel }}$ & $v_{\mathrm{obs}}$ & $v_{\text {hel }}$ & $v_{\text {obs }}$ & $v_{\text {hel }}$ & $v_{\text {obs }}$ & $v_{\text {hel }}$ & $v_{\text {obs }}$ & $v_{\text {hel }}$ \\
\hline $17-05-2016$ & $07: 22: 18.596$ & -31.90 & -15.21 & -29.59 & -12.89 & -39.18 & -22.49 & -31.56 & -14.87 & -36.48 & -19.79 & -27.20 & -10.51 \\
\hline $17-05-2016$ & 08:05:08.353 & -31.98 & -15.36 & -29.66 & -13.95 & - & - & -31.75 & -15.13 & -36.61 & -19.99 & -27.53 & -10.92 \\
\hline $17-05-2016$ & $08: 52: 35.292$ & -31.67 & -15.13 & -29.39 & -12.85 & -39.15 & -22.61 & -31.17 & -14.63 & -36.36 & -19.82 & -27.20 & -10.66 \\
\hline 11-07-2016 & $02: 33: 35.361$ & -06.85 & -15.93 & -04.61 & -13.68 & -13.99 & -23.07 & -06.42 & -15.49 & -10.98 & -20.05 & -02.02 & -11.09 \\
\hline 21-07-2016 & $03: 27: 16.562$ & -01.53 & -15.27 & 0.76 & -12.99 & -08.47 & -22.22 & 01.48 & -12.27 & -05.48 & -19.22 & 02.69 & -11.06 \\
\hline $21-07-2016$ & $04: 43: 37.414$ & -01.36 & -15.24 & 0.77 & -13.11 & -08.14 & -22.03 & -0.92 & -14.80 & -05.25 & -19.13 & 02.70 & -11.19 \\
\hline 21-07-2016 & $06: 33: 32.375$ & -1.33 & -15.33 & 0.55 & -13.46 & -8.21 & -22.21 & -0.81 & -14.82 & -5.11 & -19.11 & 2.84 & -11.18 \\
\hline $22-07-2016$ & $04: 48: 26.611$ & -01.04 & -15.37 & 01.11 & -13.22 & -08.38 & -22.71 & -0.54 & -14.87 & -05.36 & -19.69 & 03.78 & -10.55 \\
\hline 22-07-2016 & $05: 40: 15.273$ & -01.39 & -15.80 & 0.26 & -14.15 & -08.26 & -22.67 & -0.79 & -15.20 & -5.18 & -19.59 & 02.89 & -11.52 \\
\hline 22-07-2016 & $06: 33: 29.572$ & -0.88 & -15.36 & 1.11 & -13.37 & -08.66 & -23.14 & -0.44 & -14.92 & -05.12 & -19.60 & 3.48 & -11.00 \\
\hline Mean $v_{\text {hel }}$ & & & -15.40 & & -13.37 & & -22.57 & & -14.70 & & -19.60 & & -10.97 \\
\hline
\end{tabular}

Table 3. Identifications and Gaia proper motions; Gaia G and Johnson $B$ and $V$ magnitudes and membership probability.

\begin{tabular}{|c|c|c|c|c|c|c|}
\hline OGLE Name & $\mathrm{pm}_{\mathrm{RA}}$ & $\mathrm{pm}_{\text {Dec }}$ & Gmag & $V$ & $B$ & $\mathcal{P}_{\text {Member }}$ \\
\hline \multicolumn{7}{|c|}{ Present work } \\
\hline 234816 & -6.1170 & -8.3550 & 15.6950 & 16.401 & 17.781 & 0 \\
\hline 244523 & -3.0400 & -6.9400 & 15.3813 & 15.988 & 17.283 & 0 \\
\hline 244819 & 2.6380 & -6.6090 & 15.6814 & 16.306 & 17.614 & 100 \\
\hline 256289 & 2.5830 & -7.1310 & 15.3887 & 15.918 & 17.192 & 98 \\
\hline 402322 B 118 & 2.4800 & -6.5720 & 15.3817 & 16.011 & 17.331 & 100 \\
\hline 402370 & 2.4900 & -6.6590 & 15.5926 & 16.226 & 17.551 & 100 \\
\hline \multicolumn{7}{|c|}{ Stars from Barbuy et al. (2014) } \\
\hline 402361 B 107 & 2.8460 & -6.8850 & 15.3375 & 15.980 & 17.292 & 100 \\
\hline 244582 B 122 & 2.4820 & -6.0110 & 15.3401 & 16.000 & 17.354 & 99 \\
\hline $402607 \mathrm{~B} 128$ & 2.4810 & -6.4190 & 15.6001 & 16.260 & 17.621 & 100 \\
\hline 402531 B130 & 3.0100 & -6.1770 & 15.6871 & 16.300 & 17.597 & 100 \\
\hline \multicolumn{7}{|c|}{ Other stars from Barbuy et al. (2009) } \\
\hline 412752 B008 & 2.6170 & -6.4500 & 15.4115 & 15.990 & 17.402 & 100 \\
\hline B108 & 3.3340 & -6.2990 & 15.1898 & 16.290 & 18.313 & 99 \\
\hline B134 & 0.7350 & -5.0020 & 15.5082 & 16.040 & 17.367 & 0 \\
\hline 244829 F121 & 2.2580 & -7.0090 & 15.7923 & 16.400 & 17.849 & 99 \\
\hline
\end{tabular}

Notes. Stars from Barbuy et al. $(2009,2014)$ are also included.

strengths from VALD3 (Piskunov et al. 1995; Ryabchikova et al. 2015), National Institute of Standards \& Technology (NIST, Martin et al. 2002) ${ }^{3}$ and Kurucz 1993) ${ }^{4,5}$ and adopted values are given in Table C.1. For Fe I, we chose NIST values when available, otherwise they are from VALD3. In most cases, these coincide with the value from Kurucz, as can be seen in Table C.1. For Fe II, values from Meléndez \& Barbuy (2009) are used. The solar abundances were adopted from Grevesse \& Sauval (1998), including $\epsilon(\mathrm{Fe})=7.50$ for $\mathrm{Fe}$, except for $\mathrm{A}(\mathrm{O})=8.76$ for oxygen from Steffen et al. (2015).

\footnotetext{
3 https://physics.nist.gov/PhysRefData/ASD/lines_form. html

4 http://www.cfa.harvard.edu/amp/ampdata/kurucz23/ sekur.html

5 http://kurucz.harvard.edu/atoms.html
}

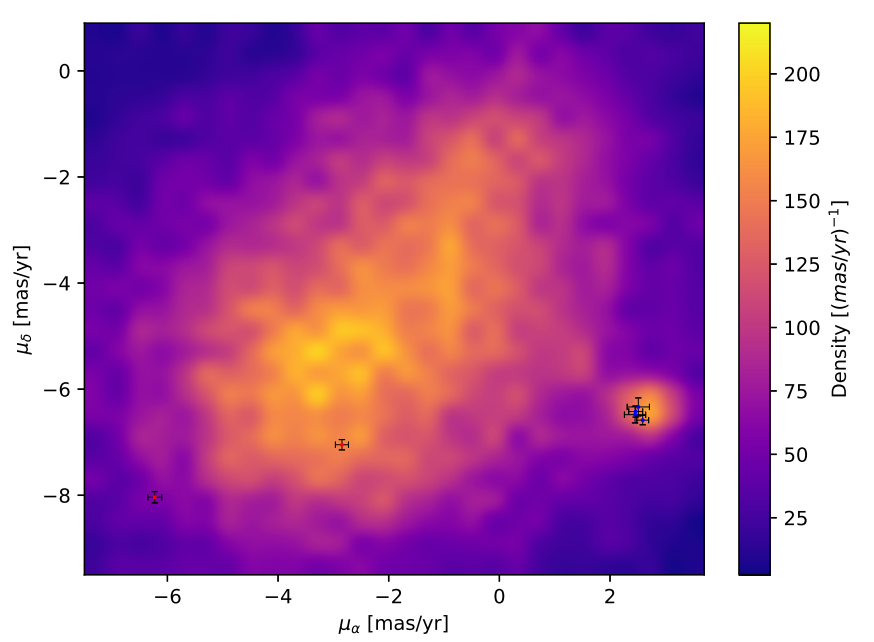

Fig. 1. Gaia proper motions at the location of NGC 6522. The background is the proper motion density plot within 8 arcmin of the cluster centre. Blue crosses are the member sample stars, and red crosses are the non-member ones.

The models were interpolated in the MARCS model atmospheres grid (Gustafsson et al. 2008). We adopted the spherical and mildly $\mathrm{CN}$-cycled set $([\mathrm{C} / \mathrm{Fe}]=-0.13,[\mathrm{~N} / \mathrm{Fe}]=+0.31)$. These models consider $[\alpha / \mathrm{Fe}]=+0.20$ for $[\mathrm{Fe} / \mathrm{H}]=-0.50$ and $[\alpha / \mathrm{Fe}]=+0.40$ for $[\mathrm{Fe} / \mathrm{H}] \leq-1.00$. The LTE abundance analysis and the spectrum synthesis calculations were performed using the code described in Barbuy et al. (2018c). The code is an update of the Meudon ABON2 code by M. Spite, continously improved along the years, which adopts local thermodynamic equilibrium (LTE). In Trevisan et al. (2011), the calculation of lines and in particular the continuum opacity calculation were cross-checked with the code by the Uppsala group BSYN/EQWI (Edvardsson et al. 1993, and updates until that date). The basic atomic line list is from VALD3 (Ryabchikova et al. 2015). Molecular lines of CN $\left(\mathrm{A}^{2} \Pi-\mathrm{X}^{2} \Sigma\right), \mathrm{C}_{2}$ Swan $\left(\mathrm{A}^{3} \Pi-\mathrm{X}^{3} \Pi\right)$, TiO $\left(\mathrm{A}^{3} \Phi-\mathrm{X}^{3} \Delta\right) \gamma$, TiO $\left(\mathrm{B}^{3} \Pi-\mathrm{X}^{3} \Delta\right) \gamma$, TiO $\alpha \mathrm{C}^{3} \Delta-\mathrm{X}^{3} \Delta$, and TiO $\beta$ $\mathrm{c}^{1} \Phi-\mathrm{a}^{1} \Pi$ systems are taken into account, as described in Barbuy et al. (2018c).

We initially adopted the photometric effective temperature and gravity, and then further constrained the temperature by 
Table 4. Identifications, coordinates, and magnitudes. $J H K_{\mathrm{s}}$ from both $2 \mathrm{MASS}$ and VVV surveys.

\begin{tabular}{|c|c|c|c|c|c|c|c|c|c|c|c|}
\hline OGLE & $2 \mathrm{MA}$ & $\alpha_{2000}$ & $\delta_{2000}$ & $V$ & $I$ & $J$ & $H$ & $K_{\mathrm{s}}$ & $J_{\mathrm{VVV}}$ & $H_{\mathrm{VvV}}$ & $K_{\mathrm{VVV}}$ \\
\hline 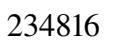 & 1 & 2.02 .265 & 1 & 16.401 & ( & 3108 & 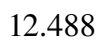 & 2 & 5 & 75 & 12305 \\
\hline & & & & & & & & & & & \\
\hline & 18 & & & & & & & & & & \\
\hline 56289 & - & & $-30: 00$ & 15. & & - & - & - & 13. & 12.4721 & 12.28 \\
\hline (2) & 5 ? & & 0 & & & 3.056 & 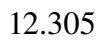 & & 12 . & & \\
\hline 2370 & $18034235-3002088$ & $18: 03: 42.35$ & $30: 02: 08.5$ & 16.226 & 14.554 & 13.391 & 12.673 & 12.550 & 13.2643 & 12.628 & \\
\hline
\end{tabular}

Table 5. Photometric stellar parameters derived using the calibrations by Alonso et al. (1999; AAM99) and Casagrande et al. (2010; C10) for $V-I$, $V-K, J-K$, bolometric corrections, bolometric magnitudes and corresponding gravity $\log g$.

\begin{tabular}{|c|c|c|c|c|c|c|c|c|c|c|}
\hline star & $\begin{array}{c}T(V-I) \\
(\mathrm{K})\end{array}$ & $\begin{array}{c}T(V-K) \\
\text { 2MASS } \\
(\mathrm{K})\end{array}$ & $\begin{array}{c}T(J-K) \\
\text { 2MASS } \\
\quad(\mathrm{K})\end{array}$ & $\begin{array}{c}T(V-K) \\
\text { VVV } \\
(\mathrm{K})\end{array}$ & $\begin{array}{c}T(J-K) \\
\text { VVV } \\
(\mathrm{K})\end{array}$ & $\begin{array}{c}\log \left(T_{\text {eff }}\right) \\
(\text { mean })\end{array}$ & $\mathrm{BC}_{\mathrm{V}}$ & $M_{\mathrm{bol}}$ & $\log g$ & Calib \\
\hline 234816 & $\begin{array}{l}4517.1 \\
4600.0\end{array}$ & $\begin{array}{l}4504.8 \\
4568.4\end{array}$ & $\begin{array}{l}4693.7 \\
4843.0\end{array}$ & $\begin{array}{l}4501.0 \\
4564.5\end{array}$ & $\begin{array}{l}4579.4 \\
4712.6\end{array}$ & $\begin{array}{l}3.655 \\
3.668\end{array}$ & $\begin{array}{l}-0.475 \\
-0.477\end{array}$ & $\begin{array}{l}0.86 \\
0.86\end{array}$ & $\begin{array}{l}2.363 \\
2.414\end{array}$ & $\begin{array}{c}\text { AAM99 } \\
\text { C10 }\end{array}$ \\
\hline 244523 & $\begin{array}{l}4792.5 \\
4854.4\end{array}$ & $\begin{array}{l}4848.0 \\
4912.6\end{array}$ & $\begin{array}{l}7391.8 \\
7372.1\end{array}$ & $\begin{array}{l}4799.3 \\
4865.1\end{array}$ & $\begin{array}{l}4806.5 \\
4969.3\end{array}$ & & $\begin{array}{l}-0.344 \\
-0.351\end{array}$ & $\begin{array}{l}0.32 \\
0.32\end{array}$ & $\begin{array}{l}2.249 \\
2.453\end{array}$ & $\begin{array}{c}\text { AAM99 } \\
\text { C10 }\end{array}$ \\
\hline 244819 & $\begin{array}{l}4858.8 \\
4917.7\end{array}$ & $\begin{array}{l}4027.3 \\
4039.6\end{array}$ & $\begin{array}{l}3340.5 \\
3115.6\end{array}$ & $\begin{array}{l}4749.2 \\
4815.7\end{array}$ & $\begin{array}{l}4746.3 \\
4902.1\end{array}$ & $\begin{array}{l}3.687 \\
3.639\end{array}$ & $\begin{array}{l}-0.319 \\
-0.325\end{array}$ & $\begin{array}{l}0.62 \\
0.61\end{array}$ & $\begin{array}{l}2.391 \\
2.199\end{array}$ & $\begin{array}{c}\text { AAM99 } \\
\text { C10 }\end{array}$ \\
\hline 256289 & $\begin{array}{l}4986.7 \\
5042.1\end{array}$ & $\begin{array}{l}- \\
-\end{array}$ & $\begin{array}{l}- \\
-\end{array}$ & $\begin{array}{l}4928.7 \\
4990.7\end{array}$ & & & $\begin{array}{l}-0.277 \\
-0.279\end{array}$ & $\begin{array}{l}0.19 \\
0.18\end{array}$ & $\begin{array}{l}2.264 \\
2.264\end{array}$ & $\begin{array}{c}\text { AAM99 } \\
\text { C10 }\end{array}$ \\
\hline 402322 & $\begin{array}{l}4715.8 \\
4782.2\end{array}$ & $\begin{array}{l}4698.8 \\
4765.6\end{array}$ & $\begin{array}{l}4638.5 \\
4780.3\end{array}$ & $\begin{array}{l}4681.9 \\
4748.8\end{array}$ & $\begin{array}{l}4686.9 \\
4835.3\end{array}$ & $\begin{array}{l}3.674 \\
3.680\end{array}$ & $\begin{array}{l}-0.376 \\
-0.383\end{array}$ & $\begin{array}{l}0.37 \\
0.37\end{array}$ & $\begin{array}{l}2.242 \\
2.266\end{array}$ & $\begin{array}{c}\text { AAM99 } \\
\text { C10 }\end{array}$ \\
\hline 402370 & $\begin{array}{l}4772.5 \\
4835.4\end{array}$ & $\begin{array}{l}4887.1 \\
4950.6\end{array}$ & $\begin{array}{l}4873.3 \\
5043.1\end{array}$ & $\begin{array}{l}4786.4 \\
4852.4\end{array}$ & $\begin{array}{l}4778.9 \\
4938.6\end{array}$ & $\begin{array}{l}3.679 \\
3.692\end{array}$ & $\begin{array}{l}-0.352 \\
-0.359\end{array}$ & $\begin{array}{l}0.57 \\
0.56\end{array}$ & $\begin{array}{l}2.341 \\
2.393\end{array}$ & $\begin{array}{c}\text { AAM99 } \\
\text { C10 }\end{array}$ \\
\hline
\end{tabular}

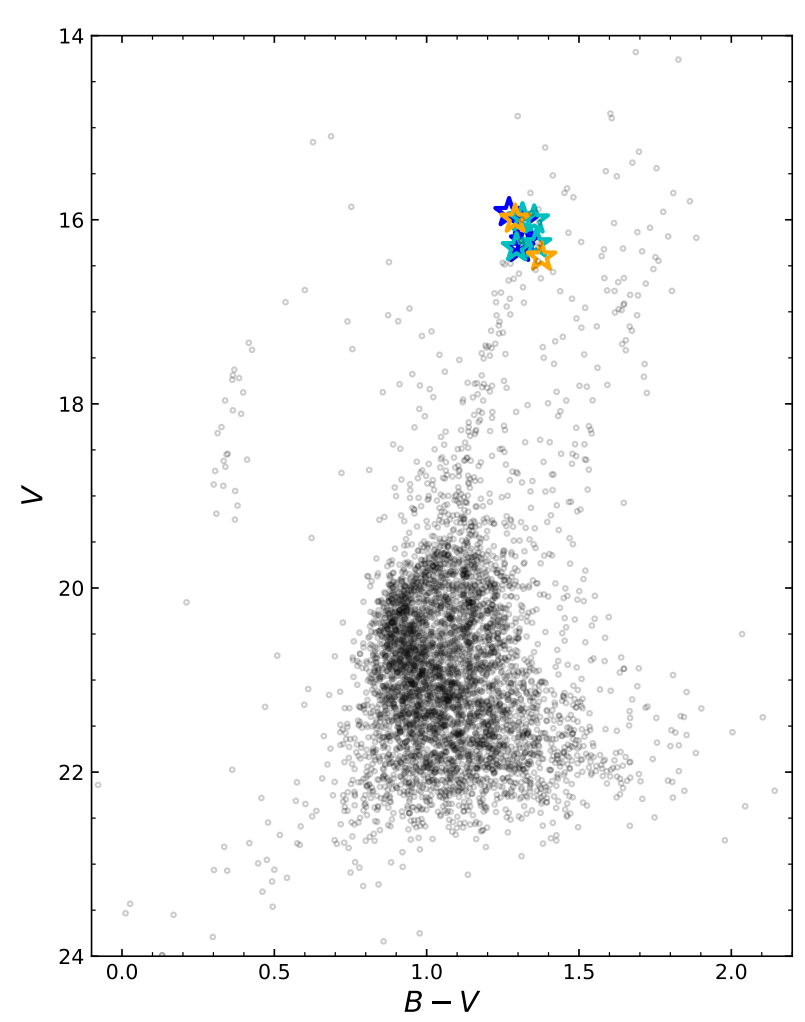

Fig. 2. NGC 6522 Colour-magnitude diagram $V$ versus $B-V$ by Piotto et al. (2002) with the location of the observed stars. Present four stars: blue. Four stars from Barbuy et al. (2014): cyan. Two non-member stars: orange. imposing an excitation equilibrium for Fe I lines and gravities by imposing ionisation equilibrium from lines of Fe I and Fe II. Microturbulence velocities $v_{t}\left(\mathrm{~km} \mathrm{~s}^{-1}\right)$ were determined by cancelling the trend of Fe I abundance versus EW. Fits to the observed spectra in regions containing the Fe II lines were carried out, as shown in Fig. 3 for star 256289. The good match of the Fe II lines indicates that these lines correspond to the equivalent widths measured, that they are not plagued by blends, and that the stellar parameters are suitable. The final spectroscopic parameters $T_{\text {eff }}, \log g,[\mathrm{Fe} \mathrm{I} / \mathrm{H}],[\mathrm{Fe} \mathrm{II} / \mathrm{H}],[\mathrm{Fe} / \mathrm{H}]$ and $v_{t}$ values are reported in Table 6. An example of excitation and ionisation equilibrium using Fe I and Fe II lines is shown in Fig. 4 for star B118.

\section{Abundance ratios}

Abundances ratios were obtained by means of line-by-line spectrum synthesis calculations compared to the observed spectra. The abundance derivation details are explained below, and the results are reported in Table 7 for $\mathrm{C}, \mathrm{N}$, and $\mathrm{O}$; and Table 8 for the $\alpha$-elements $\mathrm{O}, \mathrm{Mg}, \mathrm{Si}, \mathrm{Ca}, \mathrm{Ti}$, odd-Z elements $\mathrm{Na}, \mathrm{Al}$, and heavy elements $\mathrm{Y}, \mathrm{Zr}, \mathrm{Ba}, \mathrm{La}, \mathrm{Nd}$, and $\mathrm{Eu}$.

\subsection{Carbon, nitrogen, and oxygen}

Table 7 gives the results for $\mathrm{C}, \mathrm{N}$, and $\mathrm{O}$ abundances. The carbon abundances were estimated from the molecular $\mathrm{C}_{2}(0,1)$ Swan bandhead at $5635.5 \AA$. These bandheads are faint, and in these stars they allow us to give an upper limit only. The atomic C I $5380.3 \AA$ lines are essentially absent in these stars and cannot be 

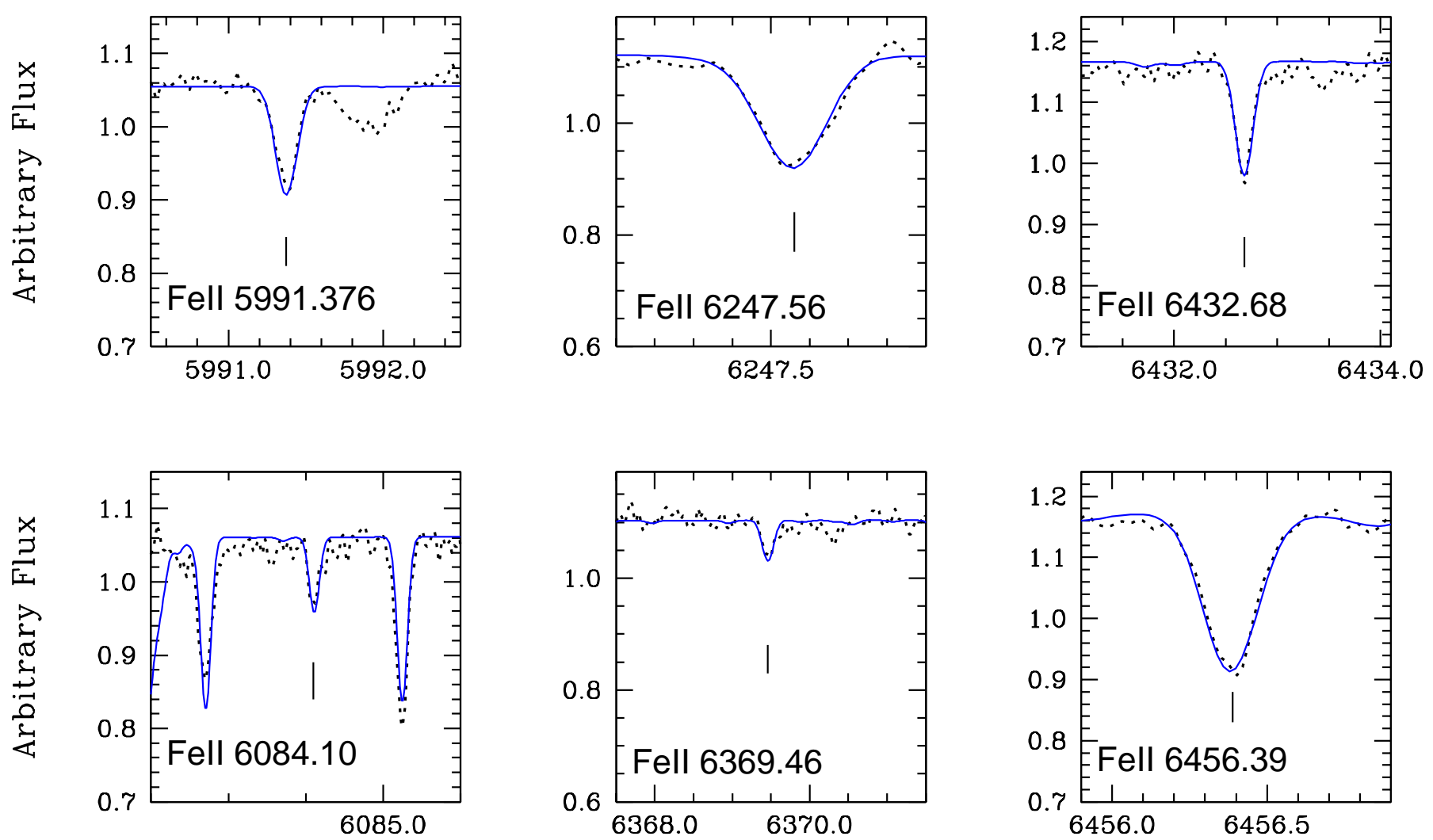

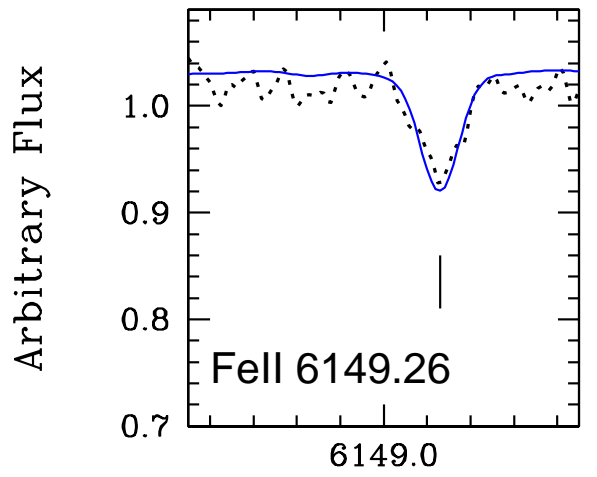

Wavelength $(\AA)$

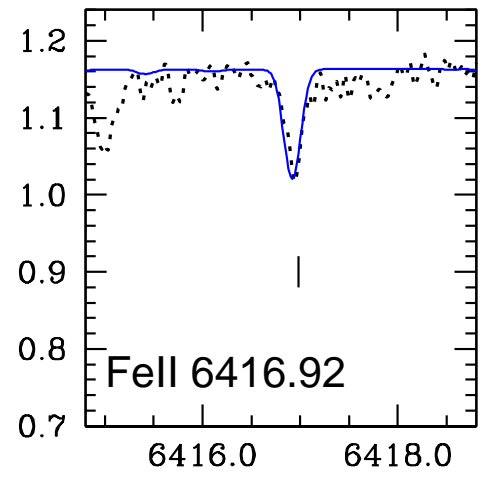

Wavelength $(\AA)$

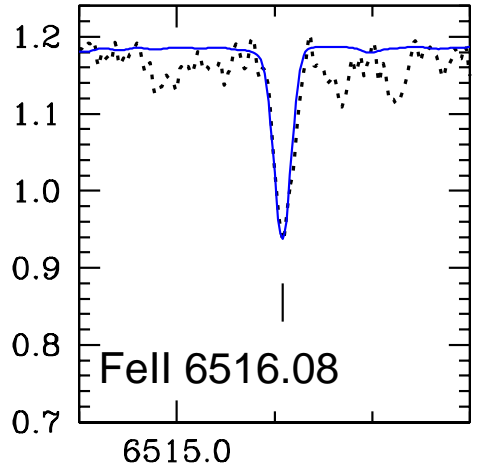

Wavelength $(\AA)$

Fig. 3. Fe II lines in star 256289.

used. The nitrogen abundance is derived from the $\mathrm{CN}(5,1)$ red system bandhead at $6332.2 \AA$. For the oxygen-forbidden line at [OI] $6300.311 \AA$, a selection among the original spectra where telluric lines did not contaminate the line was needed, since most of the observations were contaminated. A few spectra were retrieved showing a clean [OI] $6300.311 \AA$ line, and the oxygen abundance was derived. Figure 5 shows fits to $\mathrm{C}_{2}, \mathrm{CN}$ and [OI] lines for star 244819.

\subsection{Odd-Z and alpha elements}

Line-by-line abundances of the odd-Z elements $\mathrm{Na}$ and $\mathrm{Al}$ and alpha elements $\mathrm{Mg}, \mathrm{Si}, \mathrm{Ca}$, and $\mathrm{Ti}$ are reported in Table 8. Ti is an iron-peak element, but given its behaviour following the alpha-elements, it is often considered as an alpha.
Table 6. Final UVES spectroscopic parameters.

\begin{tabular}{ccccccc}
\hline \hline star & $\begin{array}{c}T_{\text {eff }} \\
(\mathrm{K})\end{array}$ & $\log g$ & {$[\mathrm{FeI} / \mathrm{H}][\mathrm{FeII} / \mathrm{H}][\mathrm{Fe} / \mathrm{H}]$} & $\begin{array}{c}v_{t} \\
\left(\mathrm{~km} \mathrm{~s}^{-1}\right)\end{array}$ \\
\hline 234816 & 4650 & 2.25 & -1.03 & -1.08 & -1.05 & 1.65 \\
244523 & 4800 & 2.00 & -1.10 & -1.12 & -1.11 & 2.30 \\
244819 & 4690 & 2.30 & -1.23 & -1.19 & -1.21 & 1.51 \\
256289 & 4770 & 2.10 & -1.12 & -1.11 & -1.11 & 1.25 \\
B118 & 4820 & 2.20 & -1.18 & -1.16 & -1.17 & 2.10 \\
402370 & 4700 & 2.20 & -1.15 & -1.16 & -1.15 & 1.15 \\
\hline
\end{tabular}

For star 234816, there is a clear overenhancement of the alpha elements as well as of the $\mathrm{r}$ element Eu. Figure 6 shows the $\mathrm{MgI}$ lines studied showing agreement for a high enhancement of $[\mathrm{Mg} / \mathrm{Fe}]=+0.7$. The odd $-\mathrm{Z}$ elements $\mathrm{Na}$ and $\mathrm{Al}$ are 

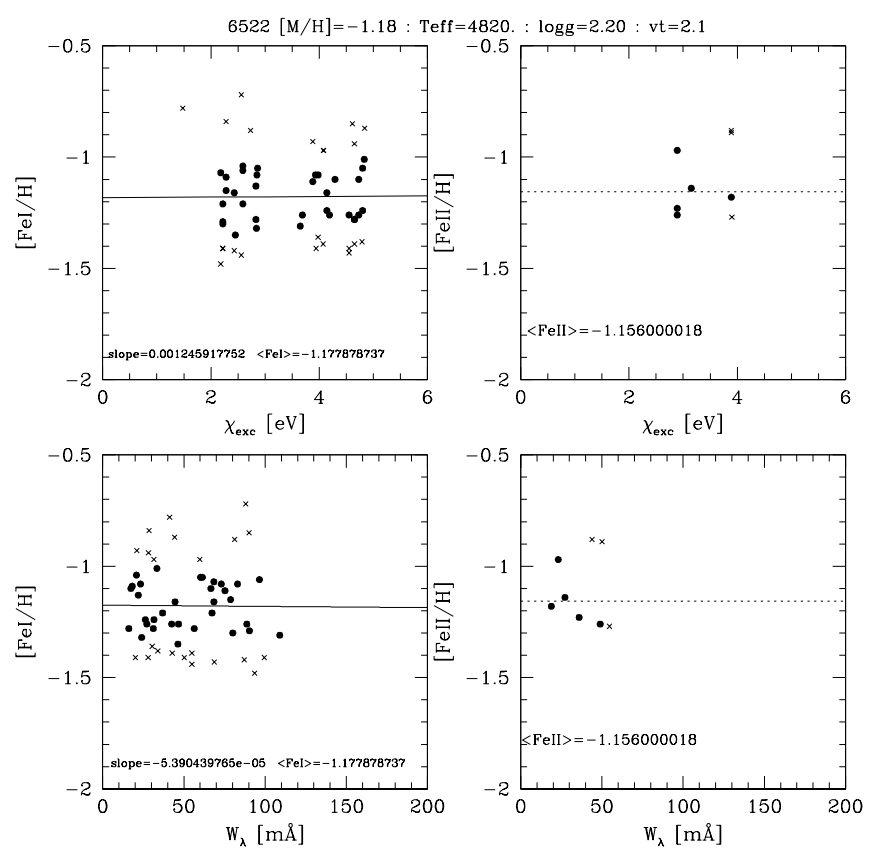

Fig. 4. Excitation and ionisation equilibrium in star B118.

Table 7. Carbon, nitrogen, and oxygen abundances derived from $\mathrm{C}_{2}$ $(0,1), \mathrm{CN}(5,1)$, and $[\mathrm{OI}]$ lines.

\begin{tabular}{lrrrrrrr}
\hline \hline line & $\lambda(\AA)$ & 234816 & 244523 & 244819 & 256289 & $\mathrm{~B} 118$ & 402370 \\
\hline $\mathrm{C}_{2}(0,1)$ & 5635.5 & $\leq+0.0$ & $\leq+0.2$ & $\leq+0.2$ & $\leq+0.2$ & $\sim+0.2$ & $\leq+0.0$ \\
$\mathrm{CN}(5,1)$ & 6332.2 & $\leq+0.8$ & $\leq+0.8$ & $\leq+1.2$ & $\leq+0.3$ & $\leq+0.8$ & $\leq+0.3$ \\
{$[\mathrm{OI}]$} & 6300.3 & +0.60 & +0.40 & +0.40 & +0.40 & - & +0.40 \\
\hline
\end{tabular}
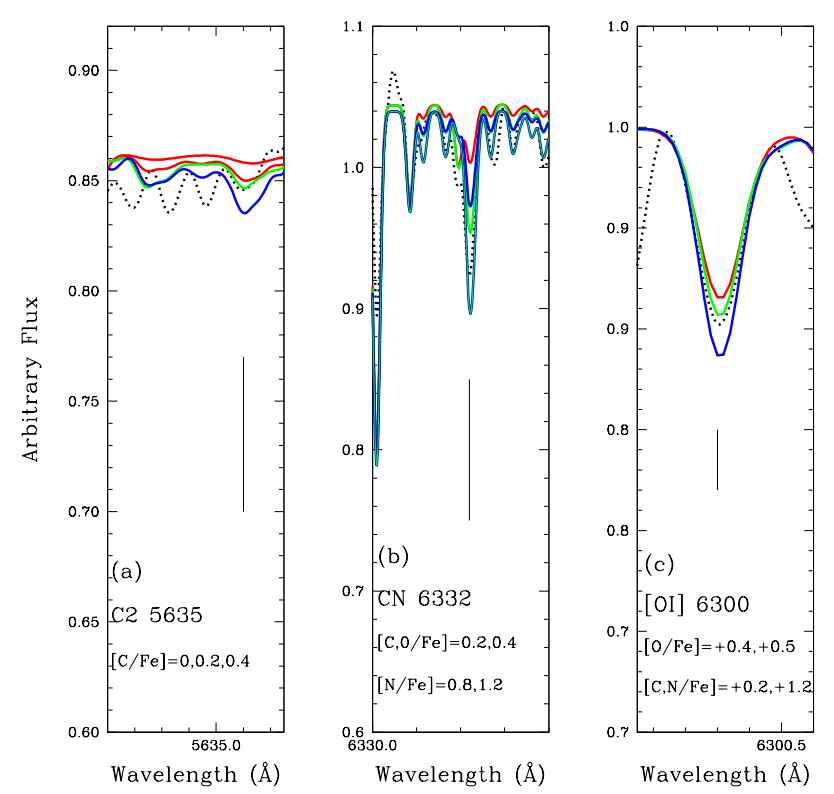

Fig. 5. C, N, and O lines in star 244819: Panel $a$ : $\mathrm{C}_{2} 5635 \AA$ A computed with $([\mathrm{C} / \mathrm{Fe}],[\mathrm{N} / \mathrm{Fe}],[\mathrm{O} / \mathrm{Fe}])=(0.0,0.8,0.4),(0.2,0.8,0.4)$ in red, $(0.2$, $1.2,0.4)$ in green, $(0.4,1.2,0.4)$ in blue. Panel $b$ : CN $6332 \AA$ computed with: $(0.2,0.8,0.4)$ in red, $(0.2,1.2,0.4)$ in green, $(0.4,0.8,0.4)$ and $(0.4,1.2,0.4)$ in blue. Panel $c$ : $[\mathrm{OI}] 6300 \AA$ computed with $(0.2,0.8$, $0.4)$ in red, $(0.2,1.2,0.4)$ in green, $(0.2,1.2,0.5)$ in blue. In all cases, black dotted lines are the observed spectra.
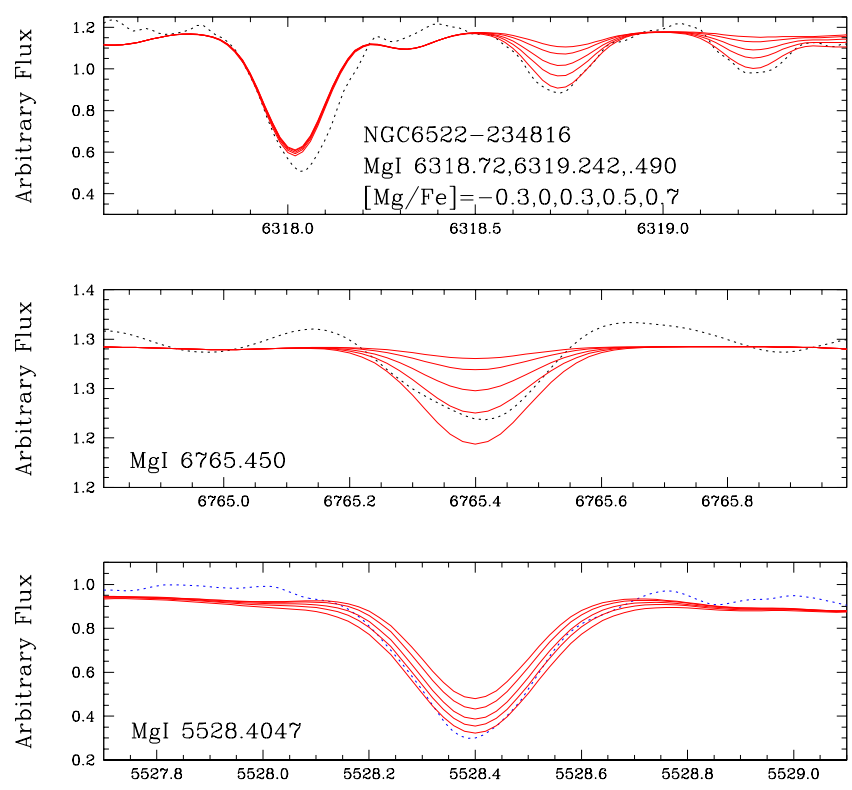

Fig. 6. Fits to $\mathrm{Mg}$ I lines in star 234816.
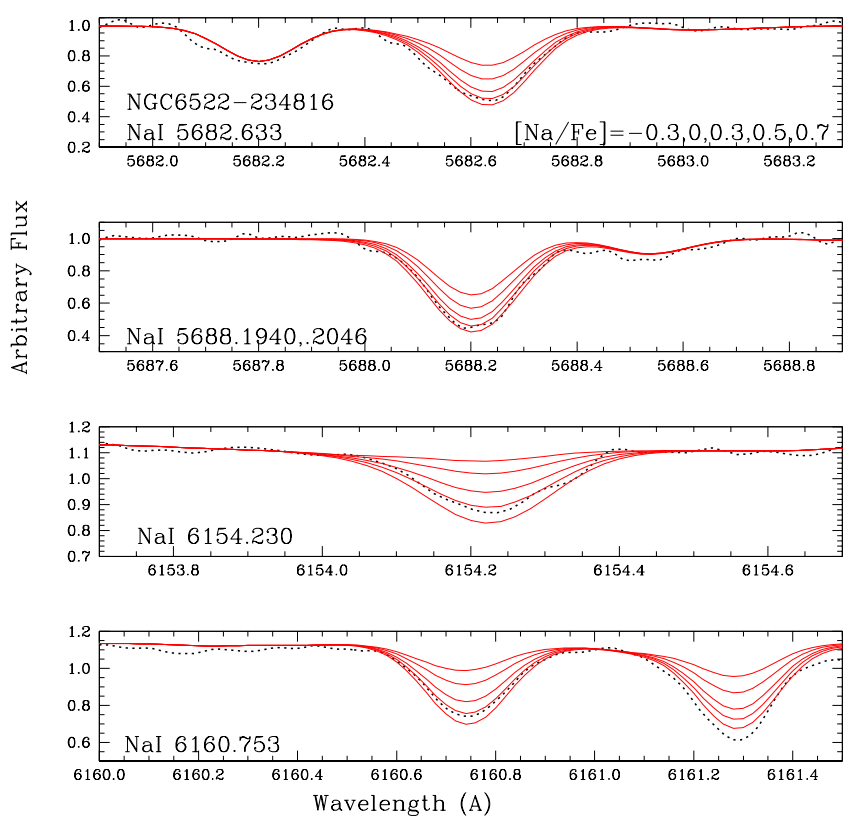

Fig. 7. Fits to Na I lines in star 234816 .

also enhanced in this star, with $[\mathrm{Na} / \mathrm{Fe}]=[\mathrm{Al} / \mathrm{Fe}]=+0.5$, as illustrated for Na lines in Fig. 7.

We exhaustively remeasured equivalent widths using other tools than IRAF, and we redetermined stellar parameters, and even with somewhat different stellar parameters were tried: models of $\left(T_{\text {eff }}, \log g,[\mathrm{Fe} / \mathrm{H}], v_{t}\right)=\left(4530 \mathrm{~K}, 2.2,-1.04,1.2 \mathrm{~km} \mathrm{~s}^{-1}\right)$ and $\left(4440 \mathrm{~K}, 2.02,-0.78,1.11 \mathrm{~km} \mathrm{~s}^{-1}\right)$ were employed in Cantelli (2019), and the overenhancement in alpha elements persists.

\subsection{Heavy elements}

Line-by-line abundances of $\mathrm{Y}, \mathrm{Zr}, \mathrm{Ba}, \mathrm{La}, \mathrm{Nd}$, and $\mathrm{Eu}$ are reported in Table 8. In Table 9 the mean abundances are reported, including results from Barbuy et al. (2014). Below we describe details on the lines of the heavy elements studied. 
A\&A 654, A29 (2021)

Table 8. Abundances in the six UVES sample stars.

\begin{tabular}{|c|c|c|c|c|c|c|c|c|c|}
\hline Species & $\lambda(\AA)$ & $\chi_{\mathrm{ex}}(\mathrm{eV})$ & $\log g f$ & 234816 & 244523 & 244819 & 256289 & B118 & 402370 \\
\hline$\overline{\mathrm{NaI}}$ & 5682.633 & 2.10 & -0.71 & +0.50 & +0.00 & +0.30 & -0.40 & +0.30 & -0.30 \\
\hline $\mathrm{NaI}$ & 5688.194 & 2.10 & -1.40 & +0.50 & -0.30 & +0.30 & -0.30 & +0.30 & -0.30 \\
\hline $\mathrm{NaI}$ & 5688.205 & 2.10 & -0.45 & +0.50 & -0.30 & +0.30 & -0.30 & +0.30 & -0.30 \\
\hline $\mathrm{NaI}$ & 6154.230 & 2.10 & -1.56 & +0.50 & +0.15 & +0.50 & -0.30 & +0.30 & +0.00 \\
\hline $\mathrm{NaI}$ & 6160.753 & 2.10 & -1.26 & +0.50 & +0.25 & +0.30 & -0.30 & +0.30 & -0.30 \\
\hline AlI & 6696.185 & 4.02 & -1.58 & +0.50 & +0.30 & +0.40 & -0.30 & +0.60 & -0.30 \\
\hline AlI & 6696.788 & 4.02 & -1.42 & +0.50 & - & +0.40 & - & - & -0.30 \\
\hline AlI & 6696.788 & 4.02 & -2.72 & +0.50 & - & +0.40 & - & - & -0.30 \\
\hline AlI & 6698.673 & 3.14 & -1.65 & +0.50 & +0.30 & +0.40 & -0.10 & +0.60 & -0.30 \\
\hline $\mathrm{MgI}$ & 5528.405 & 4.34 & -0.50 & +0.70 & +0.00 & +0.30 & +0.30 & +0.60 & +0.30 \\
\hline MgI & 6318.720 & 5.11 & -2.10 & +0.70 & +0.45 & +0.30 & +0.30 & +0.30 & +0.30 \\
\hline MgI & 6319.242 & 5.11 & -2.36 & +0.70 & +0.30 & +0.30 & +0.30 & +0.30 & - \\
\hline $\mathrm{MgI}$ & 6319.490 & 5.11 & -2.80 & +0.70 & +0.30 & +0.30 & +0.30 & +0.30 & +0.30 \\
\hline MgI & 6765.450 & 5.75 & -1.94 & +0.50 & - & - & - & - & +0.25 \\
\hline SiI & 5665.555 & 4.92 & -2.04 & +0.50 & - & - & +0.20 & +0.20 & +0.20 \\
\hline SiI & 5666.690 & 5.62 & -1.74 & +0.50 & - & - & - & +0.30 & +0.30 \\
\hline SiI & 5690.425 & 4.93 & -1.87 & +0.50 & - & - & +0.25 & +0.30 & +0.20 \\
\hline SiI & 5948.545 & 5.08 & -1.30 & +0.50 & +0.30 & +0.30 & +0.20 & +0.30 & +0.10 \\
\hline SiI & 6142.494 & 5.62 & -1.50 & +0.50 & +0.50 & +0.30 & +0.30 & +0.30 & +0.30 \\
\hline SiI & 6145.020 & 5.61 & -1.45 & +0.50 & +0.35 & +0.30 & +0.25 & +0.40 & +0.30 \\
\hline SiI & 6155.142 & 5.62 & -0.85 & +0.50 & +0.35 & +0.30 & +0.00 & +0.30 & +0.15 \\
\hline SiI & 6237.328 & 5.61 & -1.01 & +0.50 & +0.40 & - & +0.15 & +0.30 & +0.05 \\
\hline SiI & 6243.823 & 5.61 & -1.30 & +0.50 & +0.40 & +0.20 & +0.15 & +0.25 & +0.30 \\
\hline SiI & 6414.987 & 5.87 & -1.13 & +0.50 & +0.45 & - & +0.25 & +0.30 & +0.30 \\
\hline SiI & 6721.844 & 5.86 & -1.17 & +0.50 & +0.45 & - & +0.15 & +0.55 & +0.30 \\
\hline $\mathrm{CaI}$ & 5601.277 & 2.53 & -0.52 & +0.50 & -0.30 & -0.30 & -0.15 & +0.00 & +0.12 \\
\hline $\mathrm{CaI}$ & 5867.562 & 2.93 & -1.55 & +0.50 & +0.20 & +0.15 & +0.10 & -0.05 & -0.10 \\
\hline $\mathrm{CaI}$ & 6102.723 & 1.88 & -0.79 & +0.30 & -0.30 & +0.00 & +0.30 & +0.00 & +0.00 \\
\hline $\mathrm{CaI}$ & 6122.217 & 1.89 & -0.20 & +0.30 & -0.30 & +0.00 & +0.00 & +0.00 & +0.00 \\
\hline $\mathrm{CaI}$ & 6156.030 & 2.52 & -2.39 & +0.40 & +0.30 & - & +0.05 & +0.00 & +0.25 \\
\hline $\mathrm{CaI}$ & 6161.295 & 2.51 & -1.02 & +0.50 & +0.30 & +0.30 & +0.30 & +0.30 & +0.30 \\
\hline $\mathrm{CaI}$ & 6162.167 & 1.89 & -0.09 & +0.40 & -0.30 & +0.00 & +0.40 & +0.15 & +0.30 \\
\hline $\mathrm{CaI}$ & 6166.440 & 2.52 & -0.90 & +0.50 & +0.15 & +0.15 & +0.00 & +0.10 & +0.00 \\
\hline $\mathrm{CaI}$ & 6169.044 & 2.52 & -0.54 & +0.50 & +0.00 & +0.30 & +0.30 & +0.25 & +0.00 \\
\hline $\mathrm{CaI}$ & 6169.564 & 2.52 & -0.27 & +0.50 & -0.20 & +0.15 & +0.30 & +0.00 & +0.00 \\
\hline $\mathrm{CaI}$ & 6439.080 & 2.52 & +0.3 & +0.50 & -0.30 & +0.00 & +0.40 & -0.20 & +0.30 \\
\hline $\mathrm{CaI}$ & 6455.605 & 2.52 & -1.35 & +0.60 & +0.30 & - & +0.30 & +0.40 & +0.15 \\
\hline $\mathrm{CaI}$ & 6464.679 & 2.52 & -2.10 & +0.00 & - & - & +0.00 & - & - \\
\hline $\mathrm{CaI}$ & 6493.788 & 2.52 & -2.44 & +0.30 & +0.00 & +0.30 & +0.30 & -0.20 & +0.10 \\
\hline $\mathrm{CaI}$ & 6499.654 & 2.52 & -0.85 & +0.50 & +0.10 & +0.30 & +0.25 & +0.00 & +0.10 \\
\hline $\mathrm{CaI}$ & 6572.779 & 0.00 & -4.32 & - & +0.30 & +0.30 & +0.00 & +0.30 & +0.00 \\
\hline $\mathrm{CaI}$ & 6717.687 & 2.71 & -0.61 & - & +0.30 & +0.30 & $\ldots$ & +0.50 & +0.50 \\
\hline TiI & 5689.459 & 2.29 & -0.44 & +0.50 & +0.00 & +0.30 & - & +0.30 & +0.00 \\
\hline TiI & 5866.449 & 1.07 & -0.84 & +0.50 & +0.15 & +0.30 & +0.00 & +0.10 & +0.00 \\
\hline TiI & 5922.108 & 1.05 & -1.46 & +0.50 & +0.20 & +0.15 & +0.00 & +0.30 & +0.00 \\
\hline TiI & 5941.750 & 1.05 & -1.5 & +0.60 & +0.30 & +0.30 & +0.00 & +0.30 & +0.00 \\
\hline TiI & 5965.825 & 1.88 & -0.42 & +0.50 & +0.30 & +0.10 & +0.00 & +0.30 & +0.10 \\
\hline TiI & 5978.539 & 1.87 & -0.53 & +0.50 & +0.35 & +0.25 & +0.00 & +0.30 & +0.00 \\
\hline TiI & 6064.623 & 1.05 & -1.94 & +0.60 & +0.30 & +0.10 & - & - & +0.00 \\
\hline TiI & 6091.169 & 2.27 & -0.42 & +0.60 & +0.30 & +0.10 & +0.00 & +0.20 & +0.00 \\
\hline TiI & 6126.214 & 1.07 & -1.43 & +0.55 & +0.30 & +0.15 & +0.10 & +0.30 & +0.00 \\
\hline TiI & 6258.110 & 1.44 & -0.36 & +0.30 & +0.05 & -0.30 & -0.15 & -0.30 & +0.15 \\
\hline TiI & 6261.106 & 1.43 & -0.48 & +0.60 & +0.20 & +0.05 & -0.10 & +0.00 & +0.00 \\
\hline TiI & 6266.010 & 1.75 & -2.98 & - & - & - & - & - & - \\
\hline TiI & 6303.767 & 1.44 & -1.57 & +0.50 & +0.30 & +0.25 & +0.00 & +0.30 & +0.00 \\
\hline TiI & 6312.240 & 1.46 & -1.60 & +0.60 & +0.30 & +0.30 & +0.10 & +0.30 & +0.00 \\
\hline TiI & 6336.113 & 1.44 & -1.74 & +0.55 & - & +0.30 & - & +0.20 & -0.15 \\
\hline TiI & 6508.150 & 1.43 & -2.05 & - & - & - & - & - & - \\
\hline TiI & 6554.238 & 1.44 & -1.22 & +0.35 & +0.30 & +0.15 & - & +0.40 & -0.30 \\
\hline TiI & 6556.077 & 1.46 & -1.07 & +0.50 & +0.30 & +0.30 & +0.00 & +0.30 & +0.00 \\
\hline TiI & 6599.113 & 0.90 & -2.09 & +0.60 & +0.40 & +0.30 & +0.00 & +0.35 & +0.00 \\
\hline TiI & 6743.127 & 0.90 & -1.73 & +0.50 & +0.30 & +0.30 & +0.00 & +0.30 & -0.10 \\
\hline TiII & 5336.771 & 1.58 & -1.70 & +0.50 & +0.10 & +0.30 & +0.00 & - & +0.30 \\
\hline TilI & 5381.0212 & 1.57 & -2.08 & +0.50 & +0.10 & +0.30 & +0.00 & -0.30 & +0.00 \\
\hline TilI & 5418.751 & 1.58 & -2.13 & +0.50 & +0.30 & +0.50 & +0.20 & -0.20 & +0.10 \\
\hline TiII & 6491.580 & 2.06 & -2.10 & +0.35 & +0.30 & +0.30 & +0.30 & +0.40 & +0.15 \\
\hline Till & 6559.576 & 2.05 & -2.35 & +0.35 & +0.30 & +0.30 & +0.30 & +0.30 & +0.30 \\
\hline Till & 6606.970 & 2.06 & -2.85 & +0.50 & +0.30 & - & +0.20 & +0.25 & +0.15 \\
\hline Y I & 6435.004 & 0.07 & -0.82 & +0.50 & +0.80 & +0.80 & -0.15 & +0.30 & +0.30 \\
\hline Y II & 6795.414 & 1.74 & -1.19 & +0.30 & +0.50 & +1.00 & +0.30 & +0.40 & +0.00 \\
\hline $\mathrm{ZrI}$ & 6127.475 & 0.15 & -1.06 & +0.50 & - & - & +0.15 & +0.50 : & - \\
\hline $\mathrm{ZrI}$ & 6134.585 & 0.00 & -1.426 & +0.65 & - & - & +0.10 & +0.50 & - \\
\hline $\mathrm{Zr} \mathrm{I}$ & 6140.535 & 0.52 & -1.6 & - & - & - & +0.10 & - & - \\
\hline $\mathrm{ZrI}$ & 6143.252 & 0.07 & -1.1 & +0.65 & - & - & +0.10 & +0.50 & - \\
\hline Ba II & 5853.675 & 0.60 & -1.1 & +0.50 & +0.65 & +0.30 & +0.30 & +0.15 & +0.60 \\
\hline Ba II & 6141.713 & 0.70 & -0.08 & +0.50 & +0.60 & +0.30 & +0.30 & +0.00 & +0.60 \\
\hline Ba II & 6496.897 & 0.60 & -0.32 & +0.60 & +0.65 & +0.50 & +0.50 & +0.30 & +0.80 \\
\hline La II & 6262.287 & 0.40 & -1.60 & +0.40 & +1.00 & +0.40 & +0.00 & +0.60 & +0.00 \\
\hline La II & 6320.376 & 0.17 & -1.56 & +0.30 & +0.80 & +0.30 & +0.00 & +0.50 & +0.00 \\
\hline La II & 6390.477 & 0.32 & -1.41 & +0.40 & +0.90 & +0.40 & +0.00 & +0.60 & +0.10 \\
\hline Nd II & 6549.525 & 0.06 & -2.01 & +0.30 & +0.60 & - & +0.00 & +0.30 & +0.00 \\
\hline Nd II & 6740.078 & 0.06 & -1.53 & +0.00 & +0.80 & +0.30 & +0.00 & +0.30 & +0.00 \\
\hline Nd II & 6790.372 & 0.18 & -1.77 & +0.30 & +0.80 & +0.00 & +0.00 & +0.30 & -0.30 \\
\hline Eu II & 6173.029 & 1.32 & -0.86 & +0.80 & - & - & - & - & - \\
\hline Eu II & 6437.640 & 1.32 & -0.32 & +0.80 & +0.80 & +0.50 & +0.40 & +0.50 & +0.50 : \\
\hline $\mathrm{Eu}$ II & 6645.064 & 1.38 & +0.12 & +0.80 & +0.50 & +0.50 & +0.45 & +0.60 & +0.50 \\
\hline
\end{tabular}


Table 9. Mean abundances of C, N, odd-Z elements Na, Al, $\alpha$-elements O, Mg, Si, Ca, Ti, and heavy elements Y, Zr, Ba, La, and Eu.

\begin{tabular}{|c|c|c|c|c|c|c|c|c|c|c|c|c|c|c|c|c|}
\hline star & {$[\mathrm{C} / \mathrm{Fe}]$} & {$[\mathrm{N} / \mathrm{Fe}]$} & {$[\mathrm{Na} / \mathrm{Fe}]$} & {$[\mathrm{Al} / \mathrm{Fe}]$} & {$[\mathrm{O} / \mathrm{Fe}]$} & {$[\mathrm{Mg} / \mathrm{Fe}]$} & {$[\mathrm{Si} / \mathrm{Fe}]$} & {$[\mathrm{Ca} / \mathrm{Fe}]$} & {$[\mathrm{TiI} / \mathrm{Fe}]$} & {$[\mathrm{TiII} / \mathrm{Fe}]$} & {$[\mathrm{Y} / \mathrm{Fe}]$} & {$[\mathrm{Zr} / \mathrm{Fe}]$} & {$[\mathrm{Ba} / \mathrm{Fe}]$} & {$[\mathrm{La} / \mathrm{Fe}]$} & {$[\mathrm{Nd} / \mathrm{Fe}]$} & {$[\mathrm{Eu} / \mathrm{Fe}]$} \\
\hline \multicolumn{17}{|c|}{ The two non-member stars } \\
\hline 234816 & $<0.2$ & +0.80 & +0.50 & +0.50 & +0.60 & +0.66 & +0.50 & +0.42 & +0.52 & +0.45 & +0.40 & +0.60 & +0.53 & +0.36 & +0.20 & +0.80 \\
\hline 244523 & $<0.2$ & $<0.8$ & -0.04 & +0.30 & +0.40 & +0.26 & +0.40 & +0.02 & +0.26 & +0.18 & +0.65 & - & +0.63 & +0.90 & +0.70 & +0.65 \\
\hline \multicolumn{17}{|c|}{ Four stars from the present work } \\
\hline 244819 & $<0.2$ & $<1.2$ & +0.34 & +0.40 & +0.40 & +0.30 & +0.28 & +0.14 & +0.19 & +0.34 & +0.90 & - & +0.36 & +0.36 & +0.15 & +0.50 \\
\hline 256289 & $<0.2$ & $<0.3$ & -0.32 & -0.20 & +0.40 & +0.30 & +0.19 & +0.18 & +0.00 & +0.17 & +0.08 & +0.11 & +0.36 & +0.00 & +0.00 & +0.43 \\
\hline B118 & $\lesssim 0.2$ & $<0.8$ & +0.30 & +0.60 & - & +0.40 & +0.32 & +0.10 & +0.23 & +0.09 & +0.35 & +0.50 & +0.15 & +0.57 & +0.30 & +0.55 \\
\hline 402370 & $<0.0$ & $<0.3$ & -0.24 & -0.30 & +0.40 & +0.29 & +0.23 & +0.13 & -0.02 & +0.16 & +0.15 & - & +0.66 & +0.03 & -0.10 & +0.50 \\
\hline \multicolumn{17}{|c|}{ Four stars from Barbuy et al. (2014) } \\
\hline B-107 & +0.00 & - & +0.03 & +0.28 & +0.50 & +0.33 & +0.17 & +0.16 & +0.03 & +0.17 & +0.32 & +0.20 & +0.45 & +0.20 & - & +0.40 \\
\hline B-122 & -0.20 & +0.70 & +0.09 & +0.18 & +0.20 & +0.10 & +0.06 & +0.00 & +0.03 & +0.15 & +0.20 & +0.10 & +0.05 & +0.35 & - & +0.30 \\
\hline B-128 & +0.10 & +0.60 & +0.01 & +0.08 & +0.23 & +0.23 & +0.14 & +0.20 & +0.05 & +0.17 & +0.43 & +0.40 & +0.55 & +0.35 & - & +0.30 \\
\hline B-130 & +0.00 & +0.70 & +0.05 & +0.26 & +0.50 & +0.27 & +0.13 & +0.15 & +0.03 & +0.18 & +0.23 & +0.00 & +0.22 & +0.00 & - & +0.20 \\
\hline Mean & $\sim+0.14$ & +0.66 & +0.09 & +0.24 & +0.38 & +0.28 & +0.19 & +0.13 & +0.07 & +0.18 & +0.33 & +0.23 & +0.35 & +0.23 & +0.09 & +0.40 \\
\hline
\end{tabular}

Notes. The four stars analysed in Barbuy et al. (2014) are also included. For the mean values, only the eight member stars were considered.

Table 10. Heavy element abundance ratios.

\begin{tabular}{lrrrrr}
\hline \hline star & {$[\mathrm{Ba} / \mathrm{La}]$} & {$[\mathrm{Ba} / \mathrm{Eu}]$} & {$[\mathrm{La} / \mathrm{Eu}]$} & {$[\mathrm{Y} / \mathrm{Ba}]$} & {$[\mathrm{Y} / \mathrm{La}]$} \\
\hline 234816 & +0.17 & -0.27 & -0.44 & -0.13 & +0.04 \\
244523 & -0.27 & -0.02 & +0.25 & +0.02 & -0.25 \\
\hline 244819 & +0.00 & -0.14 & -0.14 & +0.54 & +0.54 \\
256289 & +0.36 & -0.07 & -0.43 & -0.28 & +0.08 \\
B118 & -0.42 & -0.40 & +0.02 & +0.20 & -0.22 \\
402370 & +0.63 & +0.16 & -0.47 & -0.51 & +0.12 \\
\hline B-107 & +0.25 & +0.05 & -0.20 & -0.13 & +0.12 \\
B-122 & -0.30 & -0.25 & +0.05 & +0.15 & -0.15 \\
B-128 & +0.20 & +0.25 & +0.05 & -0.12 & +0.08 \\
B-130 & +0.22 & +0.02 & -0.20 & +0.01 & +0.23 \\
\hline
\end{tabular}

Barium, lanthanum and europium. The hyperfine structure (HFS) for the studied lines of Ba II 5853.675, 6141.713 and $6496.897 \AA$ and Eu II $6645.064 \AA$ were taken into account. For Ba II 5853.675, we computed the splitting of lines by employing a code made available by Andrew McWilliam (McWilliam et al. 2013). For Ba II 6141.713 $\AA$ and Ba II 6496.897 $\AA$ lines, as well as for La II lines, the HFS structure was reported in Barbuy et al. (2014), and for Eu II $6645.064 \AA$ the HFS was adopted from Hill et al. (2002). For Ba II $5853.675 \AA$, the magnetic dipole $A$-factor, and the electric quadrupole $B$-factor were adopted from Biehl (1976) and Rutten (1978), as given in Table B.1. The nuclear spin is $I=1.5$ and the isotopic nuclides $\mathrm{Ba}^{138}$ and $\mathrm{Ba}^{137} \mathrm{Ba}^{136}, \mathrm{Ba}^{135}$ and $\mathrm{Ba}^{134}$ contribute with $71.7 \%$ and $11.23 \%, 7.85 \%, 6.59 \%$, $2.42 \%$, respectively (Asplund et al. 2009). The hyperfine splitting applies only to the odd- $\mathrm{Z}$ nuclides $\mathrm{Ba}^{135}$ and $\mathrm{Ba}^{137}$. The line list taking into account hyperfine structure for Ba II $5853.675 \AA$ is given in Table B.2.

Figures 8, 9, 10, 11, and 12 show, respectively, the fits to the Y I and Y II, three Ba II, La II, Nd II lines, and Eu II studied lines in the six sample stars. In Table 10 are given the ratios between $\mathrm{Ba}$ and $\mathrm{La}$ that should be produced in the same nucleosynthesis process, $\mathrm{Ba}$ and La over Eu indicating the $s$ - over $r$-process, and
$\mathrm{Y}$ over $\mathrm{Ba}$ and $\mathrm{La}$, indicating production of first peak to second peak elements.

Strontium. We carefully inspected the $\mathrm{Sr}$ lines and give the conclusions here. The line Sr I $6503.989 \AA$ is in the wing of another line and is very shallow; $\operatorname{Sr}$ I $6791.016 \AA$ is also very shallow, and only at higher $\mathrm{S} / \mathrm{N}$ it could be used (as we did in Barbuy et al. 2014). We here describe the blends contained in the Sr I $6550.244 \AA$ line in detail. In Fig. 13, we show that: a) despite the presence of several $\mathrm{TiO}$ lines from the $\gamma, \gamma^{\prime}, \alpha$, and $\beta$ systems, they are faint, given that $\mathrm{TiO}$ only gets stronger in very cool stars. To take the $\mathrm{TiO}$ lines into account, we adopted $[\mathrm{O} / \mathrm{Fe}],[\mathrm{Ti} / \mathrm{Fe}]$ from Table 9; b) There are lines of Mn II, Cr I, Ca I, Fe II, Sc II, Ni I, Si I, and Tm II, but all of these are extremely faint and do not influence the strength of the blend; c) The Nd II $6550.178 \AA$ line is not very strong, but it does contribute to the strength of the blend. We used three $\mathrm{Nd}$ II lines to derive $[\mathrm{Nd} / \mathrm{Fe}]$ for the sample stars, and the fits are presented in Fig. 11. The resulting mean $\mathrm{Nd}$ abundance is then fixed in order to compute the $\mathrm{Sr}$ abundance. In Fig. 13, we show the Nd line for the abundance derived and also for a +0.1 or +0.2 dex increase; $d$ ) The main contributors to a blend are $\mathrm{C}_{2}$ and $\mathrm{CN}$ lines. Although the $\mathrm{C}$ and $\mathrm{N}$ abundances are upper limits, by adopting these values the blend is strong. The computations were done for $[\mathrm{C} / \mathrm{Fe}]=0.2$ and $[\mathrm{N} / \mathrm{Fe}]=0.8$ (that are the upper limits given in Table 5). Since $\mathrm{C}$ and $\mathrm{N}$ are anticorrelated we compute also with $[\mathrm{C} / \mathrm{Fe}]=0.1$ and $[\mathrm{N} / \mathrm{Fe}]=0.9$ and $[\mathrm{C} / \mathrm{Fe}]=0.3$ and $[\mathrm{N} / \mathrm{Fe}]=0.7$ : the strong variation in the $\mathrm{C}_{2}$ and $\mathrm{CN}$ line strengths make the derivation of $\mathrm{Sr}$ abundance very uncertain. The identified lines are $\mathrm{C}_{2} \mathrm{Swan}$ system $\left(v^{\prime}, v^{\prime \prime}\right)=(2,5): \mathrm{R} 3(30) 6550.660, \mathrm{R} 2(31) 6550.398$ and $\mathrm{R} 1(32) 6550.296$ and $\mathrm{CN}$ red system $\left(v^{\prime}, v^{\prime \prime}\right)=(6,2)$ : Q1(22) $6550.269 \AA$; e). In conclusion, we estimate values of $[\mathrm{Sr} / \mathrm{Fe}]=$ +1.6 for star 244816 and $[\mathrm{Sr} / \mathrm{Fe}]=+0.7$ for star B118 but these cannot be considered reliable.

\subsection{Errors}

Uncertainties in spectroscopic parameters are given in Table 11 for star NGC 6522: 402370. For each stellar parameter, we 

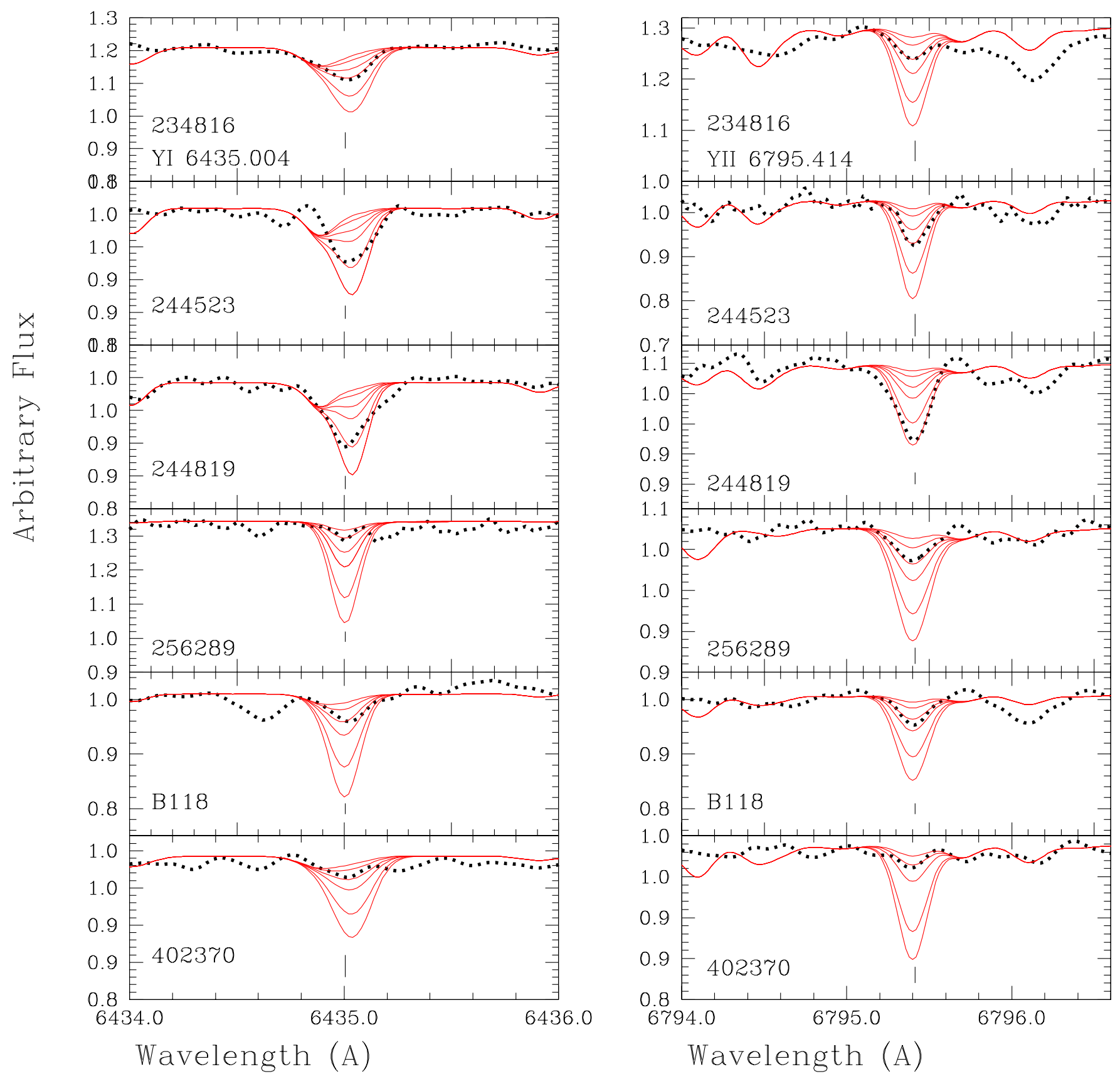

Fig. 8. Fits to Y I 6435.004, and Y II $6795.414 \AA$ in the six sample stars. Observed spectra (black dotted lines) are compared with synthetic spectra (red lines) computed for $[\mathrm{Y} / \mathrm{Fe}]=-0.3,0,0.3,0.5,0.8,1.0$.

adopted the usual uncertainties as for similar samples (Barbuy et al. 2014, 2016, 2018b): $\pm 100 \mathrm{~K}$ in effective temperature, \pm 0.2 on gravity, and $\pm 0.2 \mathrm{~km} \mathrm{~s}^{-1}$ on the microturbulence velocity. Errors were computed by employing models with these modified parameters, with changes of $\Delta T$ eff $=+100 \mathrm{~K}, \Delta \log g=$ $+0.2, \Delta v_{t}=0.2 \mathrm{~km} \mathrm{~s}^{-1}$, and recomputing lines of different elements. The error given is the abundance difference needed to reach the adopted abundances. Uncertainties due to non-LTE effects are negligible for these stellar parameters as discussed in Ernandes et al. (2018). The same error analysis and estimations can be applied to other stars in our sample. A more careful discussion is required for $\mathrm{Ba}$. The heavy element abundances for star B118 reported in Table 12 show that the abundance ratios are confirmed from one work to another, except for $\mathrm{Ba}$. This is due to the use of strong lines that fall in the saturated part of the curve of growth, where the abundance is a function of the square root of the number of atoms; the bottom of the lines reaches a maximum, and the increase of abundance causes an increase in the line wings. Therefore, abundance derivations from strong lines are in general avoided, since they are too sensitive to stellar parameters and spectral resolution. The La lines are, on the other hand, faint and they are at least not affected by the same problem.

Finally, it is important to note that the main uncertainties in stellar parameters are due to uncertainties in the effective temperature, as can be seen in Table 5. The second most important source of error are the EWs, given the limited $\mathrm{S} / \mathrm{N}$ of the spectra, which can be estimated using the formula from Cayrel (1988): $\sigma_{\mathrm{EW}}=1.5 \sqrt{F W H M \cdot \delta_{x}} /(S / N)$, where $\delta_{x}$ is the pixel size. The difference in the mean metallicities between the present work 

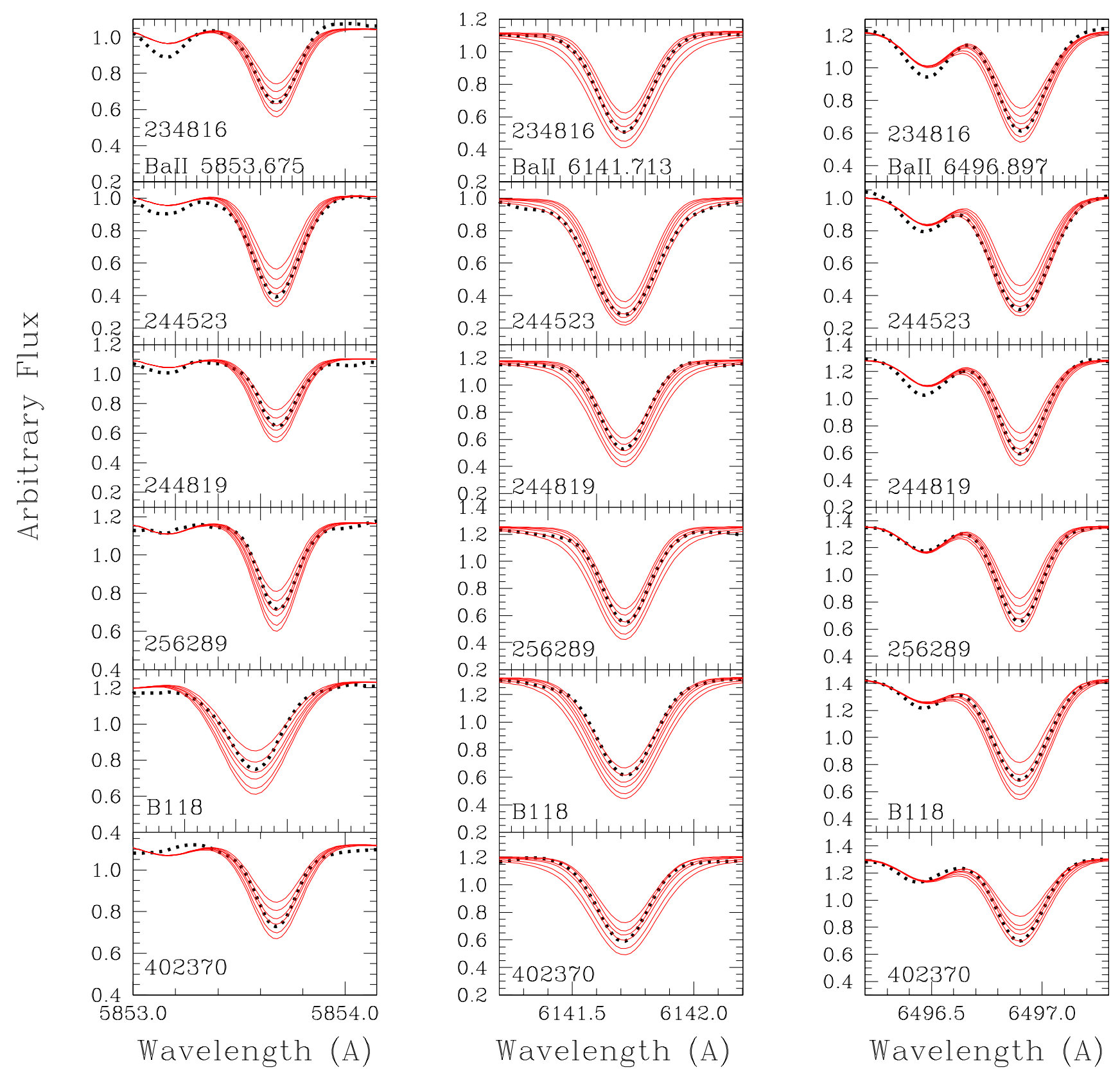

Fig. 9. Fits to Ba II $5853.675,6141.713$ and $6496.897 \AA$ in the six sample stars. Observed spectra (black dotted lines) are compared with synthetic spectra (red lines) computed for $[\mathrm{Ba} / \mathrm{Fe}]=-0.3,0,0.3,0.5,0.8,1.0$.

and Barbuy et al. (2014) are probably due to a difference in the measurements of EWs, and in particular in the placement of continua.

Comparison between results from UVES and GIRAFFE spectra. For the sample stars, we have the UVES spectra ranging from $4800-5800 \AA$, with a gap at 5777-5824 $\AA$, and the GIRAFFE spectra in the setups H11 (5597-5840) and H12 (5821-6146) only. Therefore, since most lines used for the stellar parameter analysis are in the UVES red arm, and most lines for deriving abundances are also in the UVES red arm, we cannot compare the stellar parameter derivation. Moreover, we only compare the main lines in common between the two sets of spectra, which are located at $\lambda<6142 \AA$.

By comparing the abundances for a list of lines in common between UVES and GIRAFFE, we give another indicator of uncertainty. In Table 13, we compare the abundances derived from UVES spectra to those derived from GIRAFFE spectra. The results show an excellent agreement. In order to be clear, the stellar parameters are much better derived from UVES spectra, in particular because of the measurement of the Fe II lines; whereas, given a set of stellar parameters, the abundances from the same lines at the different resolutions are both reliable.

\section{Discussion}

The inspection on abundances of heavy elements in NGC 6522 was triggered by the variation in $\mathrm{Ba}$ abundances reported in Barbuy et al. (2009). In C11, we tentatively tried to connect these abundances with the $s$-process nucleosynthesis calculations in 

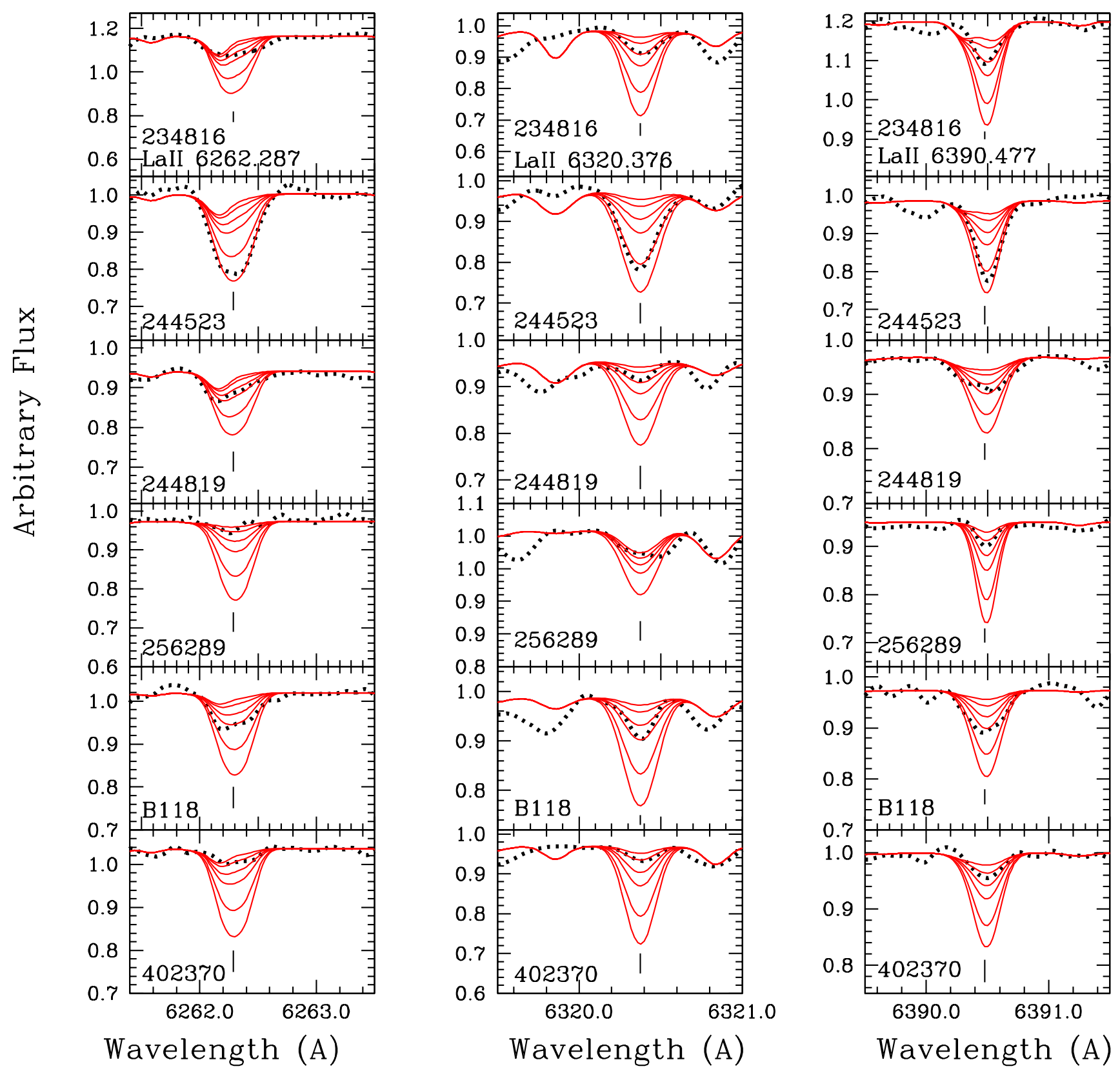

Fig. 10. Fits to La II 6262.287, 6320.376, and 6390.477 $\AA$ in the six sample stars. Observed spectra (black dotted lines) are compared with synthetic spectra (red lines) computed for $[\mathrm{La} / \mathrm{Fe}]=-0.3,0,0.3,0.5,0.8,1.0$.

spinstars first presented by Pignatari et al. (2008) and computed by Frischknecht et al. (2012). We did so for a spinstar of $40 M_{\odot}$, a metallicity of $[\mathrm{Fe} / \mathrm{H}]=-3.8$, and a rotational velocity of $V_{\text {rot }}=500 \mathrm{~km} \mathrm{~s}^{-1}$. The lower resolution data $(R \sim 22000)$ from GIRAFFE spectra were compatible with the $s$-process yields of spinstars boosted by up to four orders of magnitude with respect to a non-rotating star of the same mass and metallicity (see their Fig. 2).

A next step was presented in Barbuy et al. (2014), where the analysis of higher resolution data from UVES $(R \sim 45000)$, was studied in terms of an extended grid of spinstar models from Frischknecht et al. (2016). This paper reported enhancement of the heavy elements $\mathrm{Sr}, \mathrm{Y}, \mathrm{La}$, and $\mathrm{Ba}$ measurable in stellar spectra. The new results were shown to be compatible with expectations from massive spinstars, but allowing for other mechanisms to be invoked.
In a third step using the observations of 2012, besides the four stars observed with UVES and reported in Barbuy et al. (2014), we also identified possible new cluster member stars from the GIRAFFE spectra. In 2016, we obtained new UVES observations of six such newly identified member stars, which we analyse here.

\subsection{Analysis of the present results}

We derived a mean metallicity of $[\mathrm{Fe} / \mathrm{H}]=-1.16 \pm 0.05$, somewhat lower than the B09, B14, and Fernández-Trincado et al. (2019) values of $[\mathrm{Fe} / \mathrm{H}]=-1.0,-0.95$ and -1.04 , respectively, and closer to the value of $[\mathrm{Fe} / \mathrm{H}]=-1.15$ from Ness et al. (2014). By gathering the metallicities of the present four member stars and the other four stars from Barbuy et al. (2014), we obtain $[\mathrm{Fe} / \mathrm{H}]=-1.05 \pm 0.20$. 


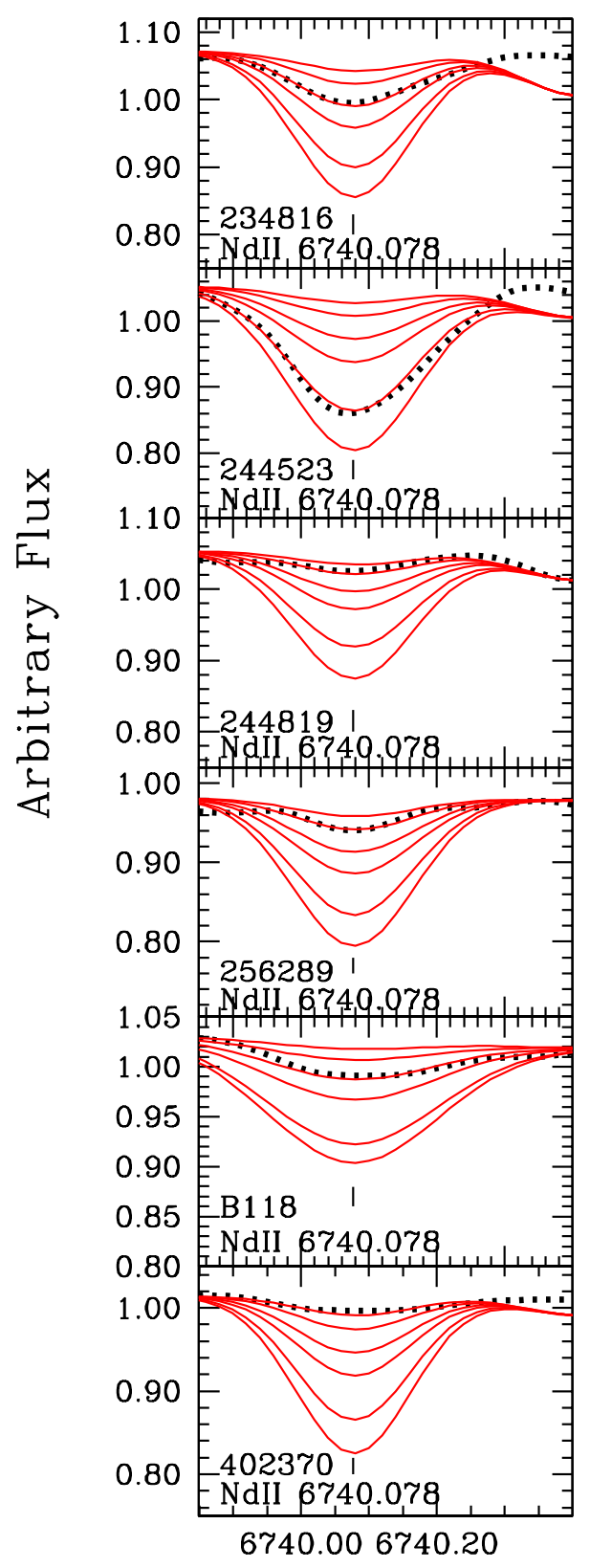

Wavelength (A)

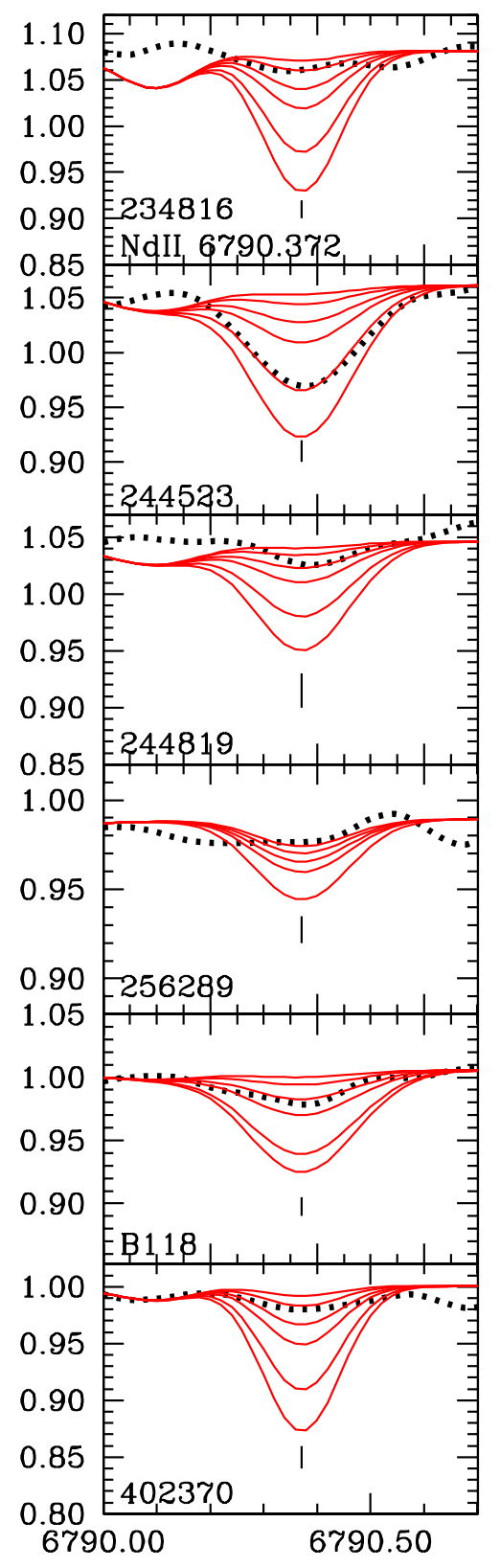

Wavelength (A)

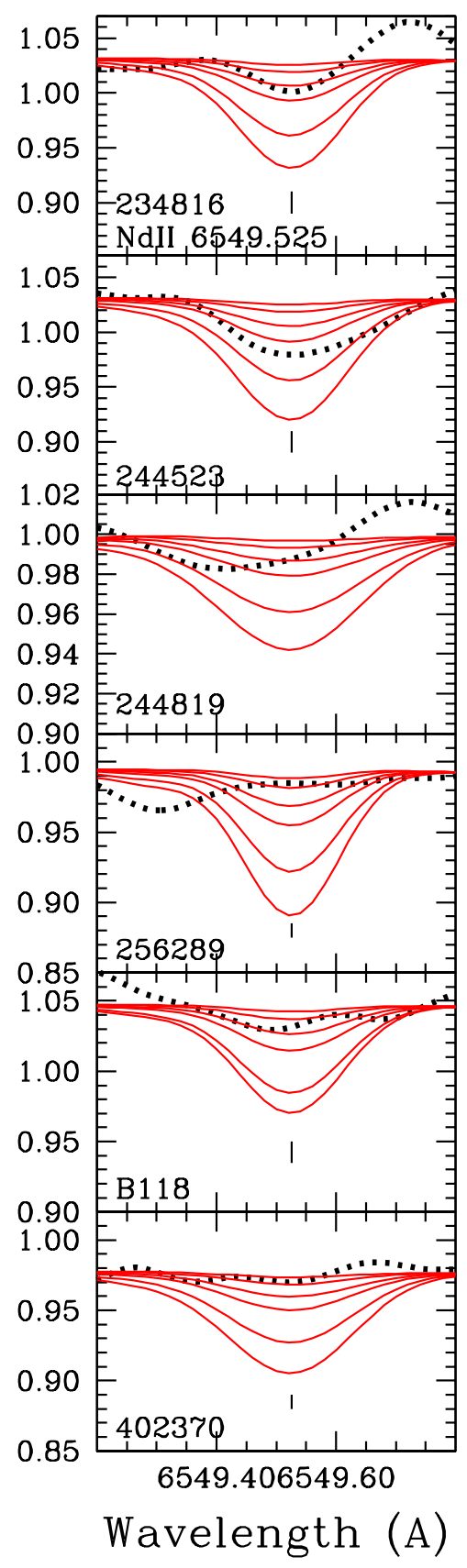

Fig. 11. Fits to Nd II 6740.078, 6790.372 and $6549.525 \AA$ in the six sample stars. Observed spectra (black dotted lines) are compared with synthetic spectra (red lines) computed for $[\mathrm{Nd} / \mathrm{Fe}]=-0.3,0,0.3,0.5,0.8,1.0$.

The mean abundances for the six sample stars, as well as the four stars studied in Barbuy et al. (2014), are reported in Table 9. In the mean we see a normal expected enhancement of the bona fide alpha elements $\mathrm{O}$ and $\mathrm{Mg}$, and a mild enhancement of $\mathrm{Si}$ and $\mathrm{Ca}$ (and $\mathrm{Ti}$, noting that $\mathrm{Ti}$ behaves as an alpha, but it is an ironpeak element). There has been evidence that the alpha elements $\mathrm{O}$ and $\mathrm{Mg}$ formed during the hydrostatic phases of massive stars nucleosynthesis are more enhanced than the other alpha elements, $\mathrm{Si}, \mathrm{Ca}$, and $\mathrm{Ti}$, which are formed predominantly during explosive nucleosynthesis (Woosley \& Weaver 1995; McWilliam 2016).

The enhancements of $\mathrm{N}, \mathrm{Na}$, and Al vary from star to star, indicating that some stars are probably second generation ones. We note that the enhancement in $\mathrm{N}$ is also due to a large scatter in its early enrichment history (e.g. Cescutti \& Chiappini 2010) as well as stellar evolution effects. In Fig. 14, we do not find a clear anti-correlation between the $[\mathrm{O} / \mathrm{Fe}]$ and $[\mathrm{Na} / \mathrm{Fe}]$ ratios in our stellar sample, such as, for instance, in NGC 6121, which is a well-populated cluster in this diagram (Carretta et al. 2009). In Fig. 14,we also show the correlated abundance signatures of $[\mathrm{Al} / \mathrm{Fe}]$ versus $[\mathrm{Na} / \mathrm{Fe}],[\mathrm{N} / \mathrm{Fe}]$ versus $[\mathrm{Na} / \mathrm{Fe}]$, and $[\mathrm{Mg} / \mathrm{Fe}]$ versus $[\mathrm{Al} / \mathrm{Fe}]$. These diagrams confirm the presence of at least two stellar populations in NGC 6522, as found by Kerber et al. (2018) from photometry.

For the heavy elements, we present the plot of abundances including the new results. In Fig. 15, we compare the present results on heavy element abundances of $\mathrm{Sr}, \mathrm{Y}, \mathrm{Zr}, \mathrm{La}, \mathrm{Ba}$, and $\mathrm{Eu}$ relative to $\mathrm{Fe}$, together with those from Barbuy et al. (2014) for 

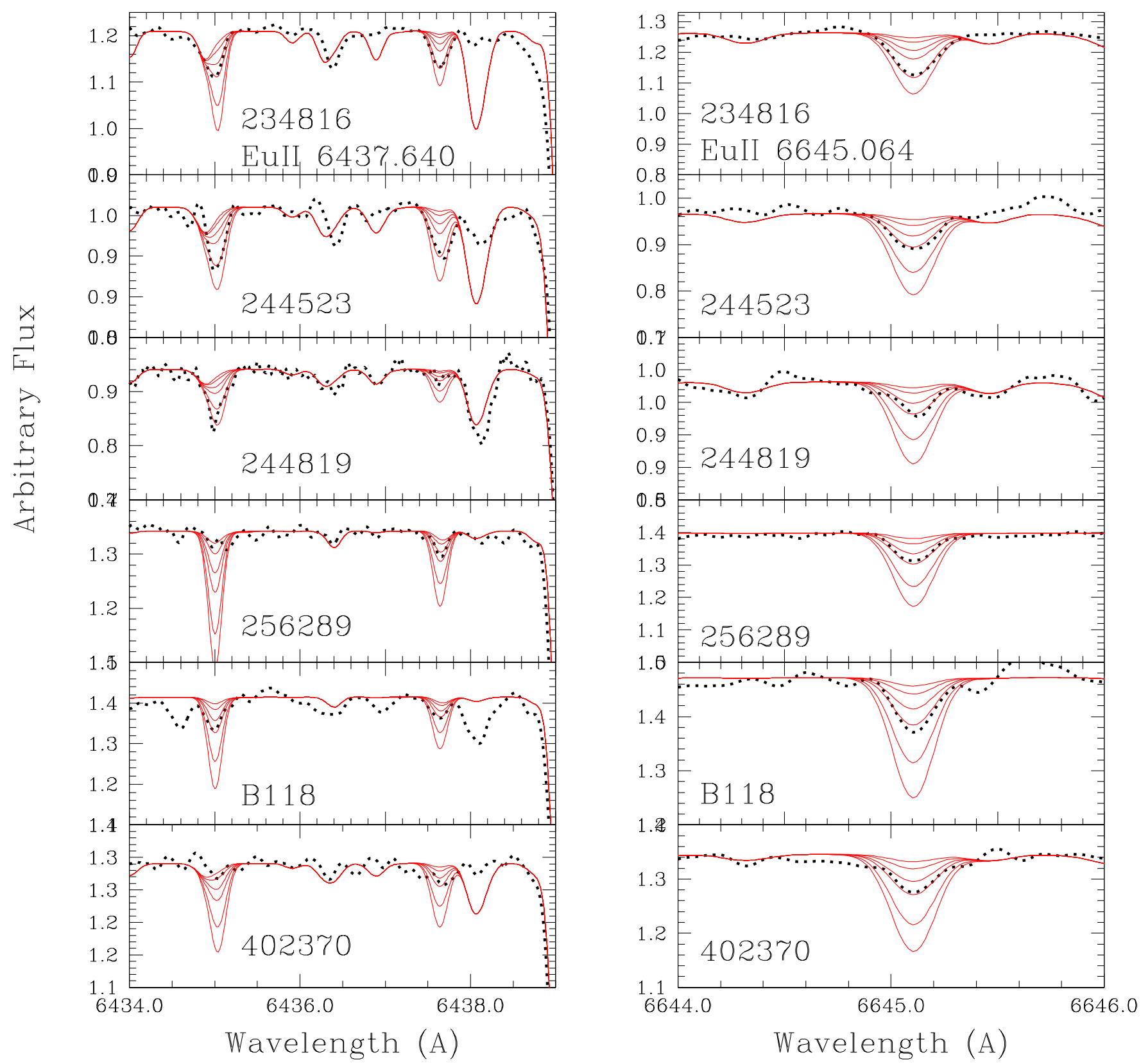

Fig. 12. Fits to Eu II 6437.650 and $6645.064 \AA$ in the six sample stars. Observed spectra (black dotted lines) are compared with synthetic spectra (red lines) computed for $[\mathrm{Eu} / \mathrm{Fe}]=-0.3,0,0.3,0.5,0.8,1.0$.

another four stars in NGC 6522. Literature abundances from field bulge red giants are from Johnson et al. (2012), for Zr, La, and Eu in Plaut's field, Siqueira-Mello et al. (2016), van der Swaelmen et al. (2016), and metal-poor giants from Howes et al. (2016) and Lamb et al. (2017). Also included are the abundances from Bensby et al. (2017 and references therein) for 39 microlensed bulge dwarfs and subgiants that are older than $9.5 \mathrm{Gyr}$, selected among their 90 stars. Finally, abundances in the bulge globular cluster HP 1 (Barbuy et al. 2016), NGC 6558 (Barbuy et al. 2018b), and M62 (Yong et al. 2014) are also shown.

From Fig. 15, the most striking feature is the abundance variation of $\mathrm{Sr}, \mathrm{Y}, \mathrm{Zr}$, and to lesser extent $\mathrm{Ba}$ and $\mathrm{La}$ at the metallicity of $[\mathrm{Fe} / \mathrm{H}] \sim-1.0$, where the bulge globular clusters are found. For Sr, we report literature data only, given the unreliability of $\mathrm{Sr}$ derivation in the present sample due to blends with $\mathrm{CN}$ and
$\mathrm{C}_{2}$ lines. We note that the spread is clearly larger in halo metalpoor stars with $[\mathrm{Fe} / \mathrm{H}] \lesssim-2.5$ (Cescutti et al. 2013; Hansen et al. 2014). For $\mathrm{Eu}$, the behaviour of $[\mathrm{Eu} / \mathrm{Fe}]$ versus $[\mathrm{Fe} / \mathrm{H}]$ is more well-defined, indicating a spread at low metallicities and a declining abundance ratio with increasing metallicities.

From Table 9 and Fig. 15, we find that a) $\mathrm{Y}$ tends to be enhanced, showing strong star-to-star variations; b) Ba tends to be enhanced, showing star-to-star variations, and c) $\mathrm{Eu}$ is enhanced similarly to the alpha elements $\mathrm{O}$ and $\mathrm{Mg}$.

As mentioned, $\mathrm{Y}$ and $\mathrm{Ba}$ variations are compatible with a large number of nucleosynthesis processes, with the member stars 244819 and B118 showing [Y/Ba] excesses of +0.54 and +0.35 , respectively. The observation of more heavy elements would be necessary to differentiate between the potential astrophysical sources. The variation in [Y/Ba] data compared with 

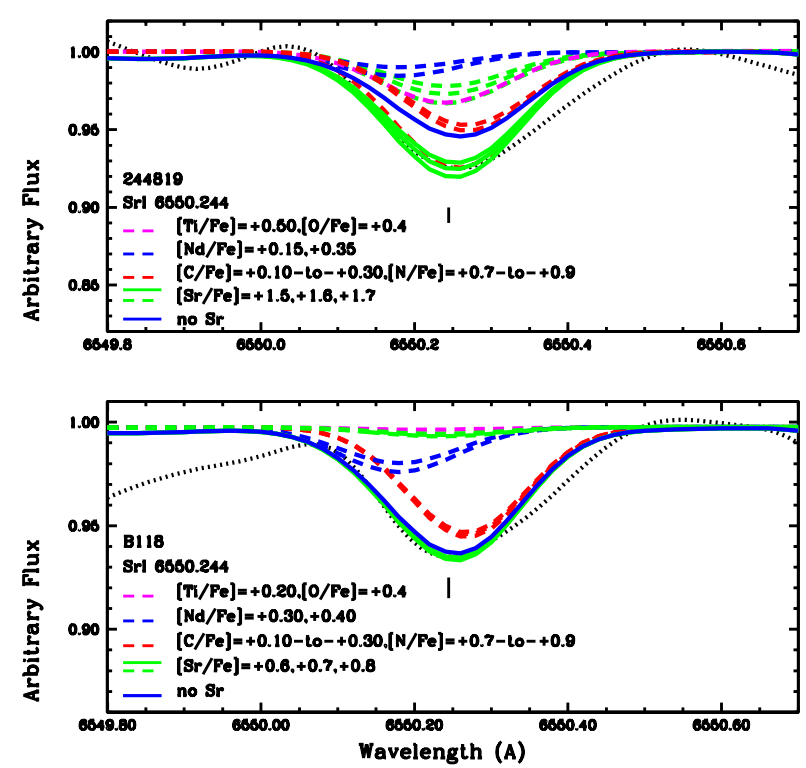

Fig. 13. Sr I $6550.244 \AA$ line in stars 244819 and B118, showing the contribution of blends to the line. Observed spectra (black dotted lines) are compared to synthetic spectra for lines as indicated in the panels. Dashed lines correspond to the contribution of a particular element only. Full lines correspond to all lines included. The values $[\mathrm{Sr} / \mathrm{Fe}]=+1.6 \pm$ 0.1 for 244819 and $[\mathrm{Sr} / \mathrm{Fe}]=+0.7 \pm 0.1$ for B118 are indicated by full green lines. These results are not considered reliable, however, due to blends.

Table 11. Abundance uncertainties for star N6522:402370, for uncertainties of $\Delta T_{\text {eff }}=100 \mathrm{~K}, \Delta \log g=0.2, \Delta v_{t}=0.2 \mathrm{~km} \mathrm{~s}^{-1}$ and corresponding total error.

\begin{tabular}{lcccc}
\hline \hline Element & $\begin{array}{c}\Delta T \\
100 \mathrm{~K}\end{array}$ & $\begin{array}{c}\Delta \log g \\
0.2 \mathrm{dex} \\
(2)\end{array}$ & $\begin{array}{c}\Delta v_{t} \\
0.2 \mathrm{~km} \mathrm{~s}^{-1}\end{array}$ & $\left(\sum x^{2}\right)^{1 / 2}$ \\
$(1)$ & -0.10 & +0.01 & +0.05 & $(5)$ \\
\hline$[\mathrm{FeI} / \mathrm{H}]$ & -0.11 & 0.11 \\
{$[\mathrm{FeII} / \mathrm{H}]$} & +0.10 & -0.07 & +0.04 & 0.13 \\
{$[\mathrm{C} / \mathrm{Fe}]$} & +0.02 & +0.02 & 0.00 & 0.03 \\
{$[\mathrm{O} / \mathrm{Fe}]$} & +0.00 & +0.05 & +0.00 & 0.05 \\
{$[\mathrm{NaI} / \mathrm{Fe}]$} & +0.05 & +0.00 & +0.00 & 0.05 \\
{$[\mathrm{AlI} / \mathrm{Fe}]$} & +0.06 & +0.00 & +0.00 & 0.06 \\
{$[\mathrm{MgI} / \mathrm{Fe}]$} & +0.00 & +0.01 & +0.00 & 0.01 \\
{$[\mathrm{SiI} / \mathrm{Fe}]$} & +0.03 & +0.00 & +0.00 & 0.03 \\
{$[\mathrm{CaI} / \mathrm{Fe}]$} & +0.08 & +0.00 & +0.01 & 0.08 \\
{$[\mathrm{TiI} / \mathrm{Fe}]$} & +0.12 & +0.01 & +0.00 & 0.12 \\
{$[\mathrm{TiII} / \mathrm{Fe}]$} & -0.05 & +0.07 & +0.00 & 0.09 \\
{$[\mathrm{YI} / \mathrm{Fe}]$} & +0.15 & +0.04 & +0.00 & 0.10 \\
{$[\mathrm{YII} / \mathrm{Fe}]$} & +0.20 & +0.15 & 0.00 & 0.25 \\
{$[\mathrm{ZrI} / \mathrm{Fe}]$} & +0.20 & -0.01 & 0.00 & 0.20 \\
{$[\mathrm{BaII} / \mathrm{Fe}]$} & +0.10 & +0.15 & -0.15 & 0.23 \\
{$[\mathrm{LaII} / \mathrm{Fe}]$} & +0.05 & +0.15 & 0.00 & 0.16 \\
{$[\mathrm{LaII} / \mathrm{Fe}]$} & +0.12 & +0.05 & 0.00 & 0.13 \\
{$[\mathrm{EuII} / \mathrm{Fe}]$} & -0.05 & +0.05 & +0.00 & 0.07 \\
\hline
\end{tabular}

Notes. The errors are to be added to reach the reported abundances.

a chemical evolution model for the Galactic bulge is shown in Fig. 16.

We note that in C11 there was no model, but instead a calculation for only one mass and that was showing the impact of rotation already. The argument was that even if that calculation was done for a very metal-poor metallicity, because in the bulge the metallicity grows quickly, we would see its effect in the very old bulge stars at $[\mathrm{Fe} / \mathrm{H}]-1.0$ as well.

In Fig. 16, we present the result of stochastic models, as presented in Cescutti et al. (2018). This can be summarised as follows. The nucleosynthesis adopted for the $s$ process from rotating massive stars comes from Frischknecht et al. (2016). In this set of yields, the $s$ process for massive stars is computed for a rotation velocity of $v_{\text {ini }} / v_{\text {crit }}=0.4$ and is composed of a grid of four stellar masses $(15,20,25$, and $40 M_{\odot}$ ) and three metallicities (solar metallicity, $10^{-3}, 10^{-5}$ ) (Cescutti \& Chiappini 2014; Cescutti et al. 2013). The model considers the enrichment produced by $r$-process events as originated from magneto-rotationally driven supernovae (MRD SNe; see Winteler et al. 2012; Nishimura et al. 2017); MRD SNe are assumed to be $10 \%$ of all the SNe II. The model also takes into account the $s$-process production from 1.5 to $3 M_{\odot}$ stars and SNIa enrichment, as in Cescutti et al. (2006). In summary, this model considers the fact that the enrichment in heavy elements takes place both in spinstars and in MRD supernovae.

A spread in abundances of these elements is observed in metal-poor halo stars (e.g. François et al. 2003; Cescutti \& Chiappini 2014; Rizzuti et al. 2021) and is expected from spinstar models (Frischknecht et al. 2012, 2016; Choplin et al. 2018; Limongi \& Chieffi 2018) and from the contribution of neutrinodriven winds in CCSNe (e.g. Roberts et al. 2010). The observed heavy element abundance ratios tend to show a higher spread of abundance ratios at the metallicity of NGC 6522 relative to the models.

However, the models presented in Fig. 16 were optimised for old field stars of the Galactic bulge, adopting the same nucleosynthesis that worked well for the Galactic halo (Cescutti et al. 2013). Although this model is not specifically made for a globular cluster, it is still useful. For instance, it shows the extension of the dispersion that the enrichment due to rotating massive stars can produce on these abundance ratios. The goal is to show that the predicted scatter is indeed compatible with the dispersion observed in NGC 6522. However, this scatter in field stars seems to appear at lower metallicities (see Barbuy et al. 2018a where our model is compared with field bulge stars). It is then plausible that other physical mechanisms are at play in the cluster evolution (involving dynamical effects, and mass loss through winds). The model for the field bulge stars would just give an idea of the mean cluster abundances but not its scatter. A detailed description of the models presented in Fig. 16, with a focus on the expected differences in the abundance ratio scatter in the bulge and halo, will be presented in Cescutti et al. (in prep.).

In order to better interpret the heavy-element abundances of star B118, in Fig. 17 we show the abundance pattern of stars 244819 and $\mathrm{B} 118$ in terms of $\mathrm{A}(\mathrm{X})-\mathrm{A}(\mathrm{Eu})$ (where $\mathrm{A}(\mathrm{X})=\log \left(N_{\mathrm{X}} / N_{\mathrm{H}}+12\right)$, in a diagram idealised by Honda et al. (2007) and Roederer et al. (2010, their Fig. 11). In this figure, $\mathrm{A}(\mathrm{X})-\mathrm{A}(\mathrm{Eu})$ versus $\mathrm{Z}$ of 244819 and B118 are compared with data from the typical r-element star CS 31082-001 (Hill et al. 2002), and the typical LEPP star HD 122563 (Honda et al. 2007; Montes et al. 2007), the identified spinstar-enriched star ROA 276 in $\omega$ Centauri (Yong et al. 2017), and the reference dwarf stars HD 94028 and HD 140283 (Peterson et al. 2020; SiqueiraMello et al. 2015). First of all, Fig. 17 indicates that 244819 and B118 are weakly enriched in r elements. Figure 18 shows $A(X)-A(E u)$ versus $Z$ for the four member stars from this paper plus the four stars from Barbuy et al. (2014) for Y, Zr, Ba, and La. It shows that the sample stars essentially follow the same pattern, 
Table 12. Heavy element results for star B118 from Barbuy et al. (2009, B09), Chiappini et al. (2011a; 2011b, C11), Ness et al. (2014, N14) and present

work.

\begin{tabular}{lrrrrrrrrrr}
\hline \hline Work & $T_{\text {eff }}$ & $\log g$ & {$[\mathrm{Fe} / \mathrm{H}]$} & $v_{\mathrm{t}}$ & {$[\mathrm{Y} / \mathrm{Fe}]$} & {$[\mathrm{Zr} / \mathrm{Fe}]$} & {$[\mathrm{Sr} / \mathrm{Fe}]$} & {$[\mathrm{Ba} / \mathrm{Fe}]$} & {$[\mathrm{La} / \mathrm{Fe}]$} & {$[\mathrm{Eu} / \mathrm{Fe}]$} \\
\hline B09 & 4700 & 2.6 & -0.84 & 1.30 & - & - & - & +1.00 & +0.50 & +0.50 \\
C11 & Same & Same & Same & Same & +0.50 & - & +1.50 & Same & Same & Same \\
N14 & 5000 & 2.25 & -1.04 & 2.45 & +0.30 & - & - & +0.30 & +0.55 & +0.40 \\
This & 4820 & 2.20 & -1.17 & 2.10 & +0.25 & +0.50 & +0.70 & +0.15 & +0.57 & +0.55 \\
\hline
\end{tabular}

Table 13. Comparison of abundances from UVES and GIRAFFE.

\begin{tabular}{|c|c|c|c|c|c|c|c|c|c|c|c|c|c|}
\hline Species & $\lambda(\AA)$ & $\begin{array}{l}234816 \\
\text { UVES }\end{array}$ & $\begin{array}{c}234816 \\
\text { GIRAFFE }\end{array}$ & $\begin{array}{c}244523 \\
\text { UVES }\end{array}$ & $\begin{array}{c}244523 \\
\text { GIRAFFE }\end{array}$ & $\begin{array}{l}244819 \\
\text { UVES }\end{array}$ & $\begin{array}{c}244819 \\
\text { GIRAFFE }\end{array}$ & $\begin{array}{c}256289 \\
\text { UVES }\end{array}$ & $\begin{array}{c}256289 \\
\text { GIRAFFE }\end{array}$ & $\begin{array}{c}\text { B118 } \\
\text { UVES }\end{array}$ & $\begin{array}{c}\text { B118 } \\
\text { GIRAFFE }\end{array}$ & $\begin{array}{c}402370 \\
\text { UVES }\end{array}$ & $\begin{array}{c}402370 \\
\text { GIRAFFE }\end{array}$ \\
\hline $\mathrm{Na} I$ & 5682.633 & +0.50 & +0.50 & +0.00 & +0.00 & +0.30 & +0.30 & -0.40 & -0.30 & +0.30 & +0.40 & -0.30 & +0.00 \\
\hline $\mathrm{NaI}$ & 5688.194 & +0.50 & +0.50 & -0.30 & +0.00 & +0.30 & +0.30 & -0.30 & -0.30 & +0.30 & +0.50 & -0.30 & +0.00 \\
\hline $\mathrm{Na} I$ & 5688.205 & +0.50 & +0.50 & -0.30 & +0.00 & +0.30 & +0.30 & -0.30 & -0.30 & +0.30 & +0.50 & -0.30 & +0.00 \\
\hline Si I & 5665.555 & +0.50 & +0.50 & - & +0.35 & - & +0.35 & +0.20 & +0.00 & +0.20 & +0.35 & +0.20 & +0.00 \\
\hline Si I & 5666.690 & +0.50 & - & - & - & - & - & - & +0.00 & +0.30 & +0.00 & +0.30 & +0.30 \\
\hline Si I & 5690.425 & +0.50 & +0.50 & - & +0.50 & - & +0.35 & +0.25 & +0.20 & +0.30 & +0.30 & +0.20 & - \\
\hline Si I & 5948.545 & +0.50 & +0.00 & +0.30 & +0.15 & +0.30 & +0.30 & +0.20 & +0.00 & +0.30 & +0.15 & +0.10 & +0.00 \\
\hline Si I & 6142.494 & +0.50 & +0.50 & +0.50 & - & +0.30 & - & +0.30 & - & +0.30 & +0.15 & +0.30 & +0.30 \\
\hline $\mathrm{CaI}$ & 5601.277 & +0.50 & +0.50 & -0.30 & +0.10 & -0.30 & +0.30 & -0.15 & +0.15 & +0.00 & +0.30 & +0.12 & +0.00 \\
\hline $\mathrm{CaI}$ & 5867.562 & +0.50 & +0.50 & +0.20 & +0.30 & +0.15 & +0.30 & +0.10 & +0.10 & -0.05 & +0.30 & -0.10 & +0.00 \\
\hline $\mathrm{Ca} I$ & 6102.723 & +0.30 & +0.30 & -0.30 & +0.00 & +0.00 & +030 & +0.30 & +0.30 & +0.00 & +0.15 & +0.00 & +0.15 \\
\hline $\mathrm{CaI}$ & 6122.217 & +0.30 & +0.30 & -0.30 & +0.00 & +0.00 & +0.30 & +0.00 & +0.15 & +0.00 & +0.00 & +0.00 & +0.00 \\
\hline Ti I & 5689.459 & +0.50 & +0.50 & +0.00 & +0.30 & +0.30 & +0.30 & - & - & +0.30 & +0.30 & +0.00 & - \\
\hline Ti I & 5866.449 & +0.50 & +0.50 & +0.15 & +0.20 & +0.30 & +0.30 & +0.00 & +0.00 & +0.10 & +0.15 & +0.00 & +0.00 \\
\hline Ti I & 5922.108 & +0.50 & +0.50 & +0.20 & +0.45 & +0.15 & +0.30 & +0.00 & +0.00 & +0.30 & +0.30 & +0.00 & +0.00 \\
\hline Ti I & 5941.750 & +0.60 & +0.50 & +0.30 & +0.50 & +0.30 & +0.30 & +0.00 & +0.20 & +0.30 & - & +0.00 & +0.00 \\
\hline Ti I & 5965.825 & +0.50 & +0.50 & +0.30 & +0.30 & +0.10 & +0.30 & +0.00 & +0.00 & +0.30 & +0.30 & +0.10 & +0.00 \\
\hline Ti I & 5978.539 & +0.50 & +0.50 & +0.35 & +0.30 & +0.25 & +0.30 & +0.00 & +0.00 & +0.30 & +0.30 & +0.00 & +0.00 \\
\hline Ti I & 6064.623 & +0.60 & +0.50 & +0.30 & +0.30 & +0.10 & +0.30 & - & +0.00 & - & +0.15 & +0.00 & +0.00 \\
\hline Ti I & 6091.169 & +0.60 & +0.50 & +0.30 & +0.30 & +0.10 & +0.30 & +0.00 & - & +0.20 & +0.30 & +0.00 & +0.00 \\
\hline Ti I & 6126.214 & +0.55 & +0.50 & +0.30 & +0.30 & +0.15 & +0.30 & +0.10 & +0.00 & +0.30 & +0.30 & +0.00 & +0.00 \\
\hline Zr I & 6127.475 & +0.50 & +0.50 & - & +0.30 & - & - & +0.15 & +0.30 & $+0.50:$ & $+0.50:$ & - & - \\
\hline Zr I & 6134.585 & +0.65 & +0.50 & - & +0.50 & - & - & +0.10 & - & +0.50 & - & - & - \\
\hline Zr I & 6140.535 & - & - & - & - & - & - & +0.10 & - & - & - & - & - \\
\hline Zr I & 6143.252 & +0.65 & +0.50 & - & - & - & - & +0.10 & - & +0.50 & +0.30 & - & - \\
\hline Ba II & 5853.675 & +0.50 & +0.50 & +0.50 & +0.60 & +0.30 & +0.30 & +0.30 & +0.30 & +0.00 & +0.20 & +0.60 & +0.30 \\
\hline Ba II & 6141.713 & +0.50 & +0.40 & +0.40 & - & +0.30 & - & +0.30 & - & -0.30 & -0.30 & +0.60 & +0.15 \\
\hline
\end{tabular}

whereas 244819 and B118 show a larger abundance difference between first peak and second peak elements.

Spite et al. (2018) suggested that the heavy element enrichment should take place first due to a pure $r$ process, followed by an enrichment of first-peak elements only, and that this second mechanism would be detectable only in weak- $r$-process stars. On the other hand, spinstars could be progenitors of magnetorotational supernovae, but in case the conditions do not allow $r$-process elements to form in the final explosion, it is also possible that we can only observe the signature of the $s$-process production in spinstars today.

There are a number of points that it is important to consider in our analysis. First of all, the s-process efficiency in spinstars varies greatly if we consider different theoretical stellar yields. While for instance the $s$-process production in models by Frischknecht et al. (2016) and Choplin et al. (2018) would stop in the Ba mass region, in models by Limongi \& Chieffi (2018) heavier elements up to lead could be produced. This uncertainty of course affects Galactic chemical evolution (GCE) predictions (e.g. Cescutti et al. 2013; Rizzuti et al. 2019, 2021; Prantzos et al. 2020). Additionally, CCSNe generated from slowly rotating progenitors or spinstars can also eject other nucleosynthesis components made before the SN explosion (similarly to the intermediate neutron capture process or i process - see e.g. Roederer et al. 2016 and Banerjee et al. 2018) or by explosion (similarly to the zoo of neutrino-driven wind components - see e.g. Qian \& Wasserburg 2008; Farouqi et al. 2009; Roberts et al. 2010; Arcones \& Montes 2011). As we mentioned earlier, all of these processes may contribute to the production of $\mathrm{Sr}, \mathrm{Y}$, and $\mathrm{Zr}$, while at low metallicities the i process can potentially produce elements across the whole mass region below and beyond $\mathrm{Fe}$, including $\mathrm{Sr}, \mathrm{Y}, \mathrm{Zr}$, and $\mathrm{Ba}$, in different types of stars (e.g. Abate et al. 2016; Roederer et al. 2016; Clarkson et al. 2018; Banerjee et al. 2018). For instance, the high $[\mathrm{Ba} / \mathrm{La}]$ in stars 256289 , 402370, B-107, B-128, and B-130 would be compatible with the i process (see e.g. Hampel et al. 2016). 

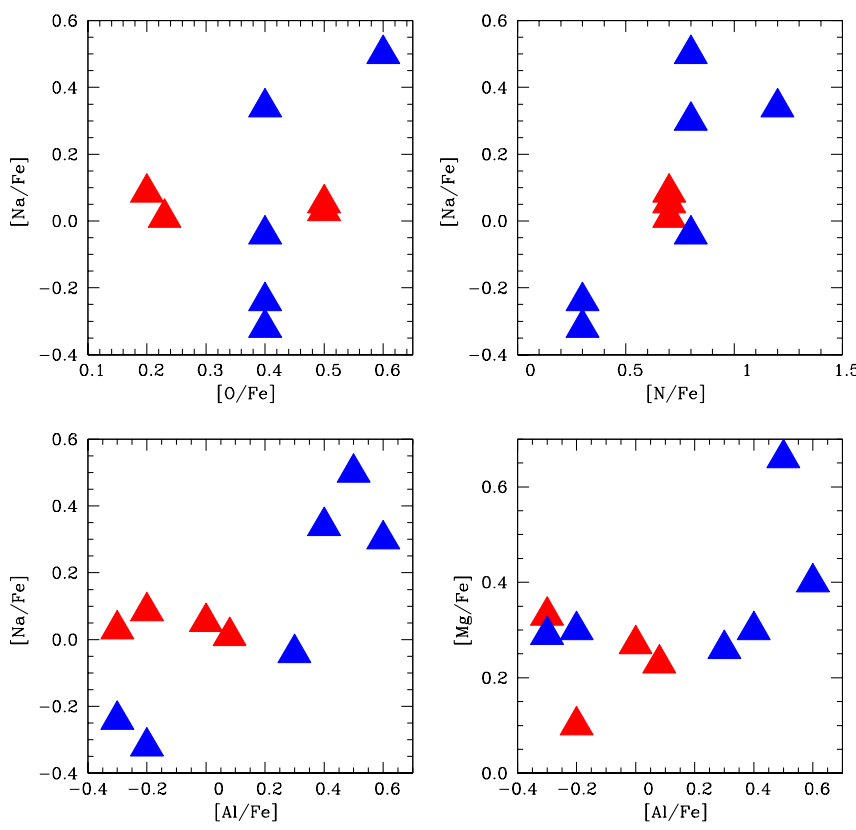

Fig. 14. $[\mathrm{Na} / \mathrm{Fe}]$ versus $[\mathrm{O} / \mathrm{Fe}]$ anti-correlation in NGC 6522 stars compared with stars in NGC 6121 ; and $[\mathrm{Na} / \mathrm{Fe}]$ versus $[\mathrm{N} / \mathrm{Fe}],[\mathrm{Al} / \mathrm{Fe}]$ and $[\mathrm{Mg} / \mathrm{Fe}]$ versus $[\mathrm{Al} / \mathrm{Fe}]$ correlations. Symbols: blue-filled triangles represent the present results for NGC 6522; red-filled triangles denote results for NGC 6522 from Barbuy et al. (2014).

Alternative possibilities of neutron-capture element enrichment are the magneto-rotationally driven explosions of corecollapse supernovae (Winteler et al. 2012), or $s$ process taking place in asymptotic giant branch (AGB) stars and subsequent mass transfer within a binary system (e.g. Cristallo et al. 2015). A study on possible nucleosynthesis processes is given in Hansen et al. (2014).

This makes the observation of more elements per stellar target and at high resolution of different stars in globular clusters such as NGC 6522 crucial. Within this scenario the large star-tostar variations of heavy-element enrichment could be a natural outcome of an intrinsic scatter of $s$-process efficiencies in spinstars or the varying contribution of different processes active before and during SN explosions in massive stars. On the other hand, when abundances of several heavy elements are available it becomes possible to disentangle the dominant nucleosynthesis component(s) that made the whole observed abundance pattern (see e.g., Roederer et al. 2016; Peterson et al. 2020). Stars in NGC 6522 are carriers of the same signatures of the nucleosynthesis processes active in the early galaxy and observed in halo stars, even if they are more metal-rich as a result of a steeper age-metallicity relation in the Galactic bulge (as suggested in C11).

\subsection{The two non-member stars 234816 and 244523}

The stars 234816 and 244523 have the correct magnitudes, radial velocities and metallicities to be members of NGC 6522 . However, the Gaia proper motions reported in Table 3 rule out their membership.

Could these two stars be former members that are evaporating from the cluster? Madrid et al. (2017) studied evaporation rates as a function of galactocentric distance $R_{\mathrm{GC}}$ and time and predicted a very high evaporation at low $R_{\mathrm{GC}}$ due to the strong tidal field in the central parts of the Galaxy. NGC 6522 is estimated to have a mass of $5.93 \times 10^{4} M_{\odot}$ (Gnedin \& Ostriker 1997), which is not high for a globular cluster. It is located at $R_{\mathrm{GC}} \approx 1 \mathrm{kpc}$ and has an age above $12.1-12.4 \mathrm{Gyr}$ using Dotter et al. (2008) isochrones and even older (with 14.114.2 Gyr) using BaSTI isochrones (Kerber et al. 2018). It can be seen from Fig. 6 of Madrid et al. (2017) that the evaporation rate in a bulge cluster like NGC 6522 should be extremely high. It is therefore acceptable to suggest that the two stars could be evaporating from the cluster. However, a more detailed orbital calculation would be needed to check this possibility, such as the one carried out by Hanke et al. (2020). In particular, star 234816 has different alpha-element abundances and should not be a member.

\section{Conclusions}

We derived a mean metallicity of $[\mathrm{Fe} / \mathrm{H}]=-1.16 \pm 0.05$ from the four sample stars. Combined with the other four stars from Barbuy et al. (2014), the result is a mean metallicity of $[\mathrm{Fe} / \mathrm{H}]=$ $-1.05 \pm 0.20$.

Among the six stars analysed, two of them are indicated to be non-members from Gaia proper motions; still, they have the correct magnitude, radial velocity, and metallicity to be members. Only a fraction of about $0.5 \%$ of stars in the Galactic bulge have metallicities below $[\mathrm{Fe} / \mathrm{H}] \lesssim-1.0$ (Barbuy et al. 2018a). Therefore, we suggest that these stars could be evaporating from the cluster; but even so, we do not include their abundances in the discussion below. Star 244523 has abundances compatible with the member stars. Star 234816 shows different high alpha-element abundances, and it could be an intruder; hence, it could have been a bulge star with the correct magnitude and metallicity to be considered a member before we had Gaia measurements, but it was eventually revealed as a non-member star.

For the present results on the four confirmed member stars, together with those by Barbuy et al. (2014), the alpha-elements show enhancements of $[\mathrm{O} / \mathrm{Fe}]=+0.38,[\mathrm{Mg} / \mathrm{Fe}]=\approx+0.28$, $[\mathrm{Si} / \mathrm{Fe}] \approx+0.19$, and $[\mathrm{Ca} / \mathrm{Fe}] \approx+0.13,[\mathrm{Ti} / \mathrm{Fe}] \approx+0.13$. A higher enhancement in $\mathrm{O}$ and $\mathrm{Mg}$, and a lower one in $\mathrm{Si}, \mathrm{Ca}$, and $\mathrm{Fe}$ can be explained by their formation in hydrostatic conditions for the former, and in explosive nucleosynthesis for the latter (e.g. Woosley \& Weaver 1995; McWilliam 2016).

The $r$-process element Eu is enhanced by $[\mathrm{Eu} / \mathrm{Fe}]=+0.40$. The $\alpha$-element enhancements in $\mathrm{O}$ and $\mathrm{Mg}$, together with that of the $r$-process element $\mathrm{Eu}$, are indicative of a fast early enrichment by type II supernovae. With regard to the indicators of multiple stellar populations, we suggest that Na shows an anticorrelation with $\mathrm{O}$, and more clearly a correlation with $\mathrm{N}$ and $\mathrm{Al}$, whereas $\mathrm{Mg}$ and $\mathrm{Al}$ are also correlated.

A main objective of this study is the verification of the enhancement of s-element abundances, and the possibility of an early enrichment by spinstars. The neutron-capture elements typically indicated as $s$-process elements are enhanced with $[\mathrm{Y} / \mathrm{Fe}]=+0.33,[\mathrm{Zr} / \mathrm{Fe}]=+0.23,[\mathrm{Ba} / \mathrm{Fe}]=+0.35$, and $[\mathrm{La} / \mathrm{Fe}]=+0.23$. In addition to this observation we find the following:

(a) There are significant relative abundance variations between neutron-capture elements, where [Y/Ba] is particularly enhanced in two stars.

(b) $[\mathrm{Ba} / \mathrm{Eu}]=-0.14,-0.07,-0.40,+0.16$, in the four member stars, as a measure of the $s$ - to $r$-process nucleosynthesis, tends to be slightly below solar. This result is still compatible 

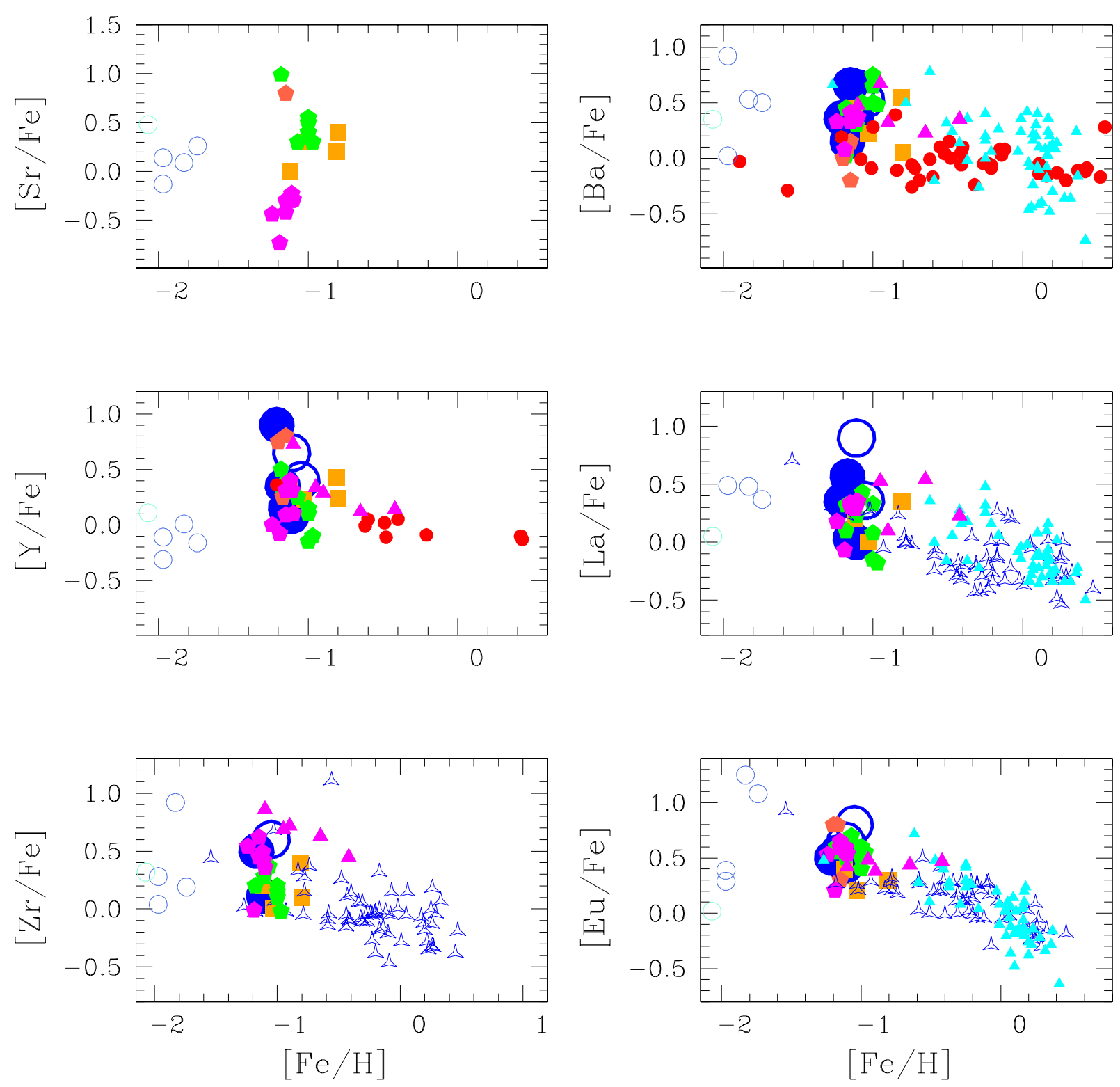

Fig. 15. $[\mathrm{Sr}, \mathrm{Y}, \mathrm{Zr}, \mathrm{Ba}, \mathrm{La}, \mathrm{Eu} / \mathrm{Fe}]$ versus $[\mathrm{Fe} / \mathrm{H}]$ in bulge stars. Symbols: large, blue-filled circles mark present results of NGC 6522 member stars; large, blue open circles represent the present results of non-member stars. Field stars: blue open triangles represent red giants by Johnson et al. (2012); magenta-filled triangles represent red giants by Siqueira-Mello et al. (2016); cyan-filled triangles denote red giants by van der Swaelmen et al. (2016); red-filled circles show dwarfs by Bensby et al. (2017); light blue open circles mark metal-poor giants by Howes et al. (2016); acquamarine open circles show metal-poor giants by Lamb et al. (2017). Globular cluster stars: orange-filled squares show NGC 6522 (Barbuy et al. 2014); green-filled pentagons mark HP 1 (Barbuy et al. 2016); tomato-filled pentagons represent NGC 6558 (Barbuy et al. 2018c); magenta-filled pentagons show M62 (Yong et al. 2014).

with the interpretation given in B09 and C11 that their production cannot be attributed to the $r$-process only, as first suggested by Truran (1981) for very old stars.

As discussed in C11, the presence of $s$-process element enhancements in very old stars could be due to an $s$-process enrichment of the primordial matter from which the cluster formed, processed in spinstars (e.g. Frischknecht et al. 2016). Alternatively, the production of heavy elements could be due to a combination of different nucleosynthesis processes, in particular for the atomic mass region of $\mathrm{Sr}$. Another possibility would be to have spinstars producing the $s$-process elements during its hydrostatic phase and producing the $r$-process elements at the supernova explosion, and therefore to be the source of both. This is possible if the spinstars rotate fast enough to produce an MHD explosion with the right conditions to produce an $r$ process (Nishimura et al. 2017 and references therein). However, within this scenario it is extremely uncertain to predict the observed relative contribution of the $s$-process and $r$-process elements, since the $r$-process-rich material could be ejected asymmetrically and/or could carry a large range of efficiency in $r$-process production. Therefore, a possible outcome could be that the final enrichment produced by such a spinstar and magnetic-rotationally driven (MRD) $\mathrm{SN}$ is dominated by the $r$-process signature, because of higher yields of the MRD SNe compared to those of the $s$ process (Spite et al. 2018). On the other hand, the enrichment of the local interstellar medium could also be $s$-process rich, depending on the spatial distribution of different nucleosynthesis products in the SN ejecta. 

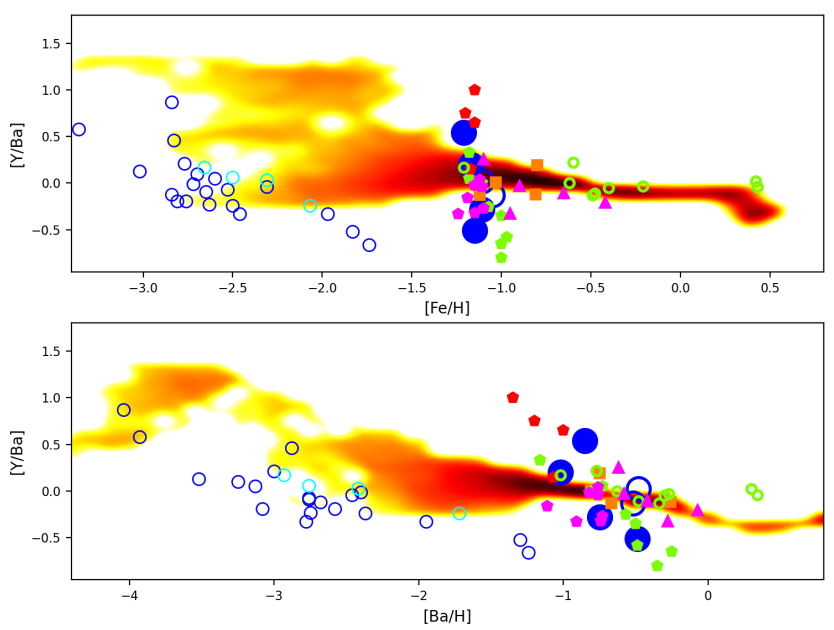

Fig. 16. $[\mathrm{Y} / \mathrm{Ba}]$ versus $[\mathrm{Fe} / \mathrm{H}]$ (upper panel) and $[\mathrm{Y} / \mathrm{Ba}]$ versus $[\mathrm{Ba} / \mathrm{H}]$ (lower panel). Symbols: Large blue-filled circles show present results on NGC 6522 member stars; large blue open circles show present results on non-member stars. Field stars: magenta-filled triangles denote red giants by Siqueira-Mello et al. (2016); green open circles mark dwarfs by Bensby et al. (2017); light blue open circles show metal-poor giants by Howes et al. (2016); acquamarine open circles represent metal-poor giants by Lamb et al. (2017). Globular cluster stars: orange-filled squares show NGC 6522 (Barbuy et al. 2014; green-filled pentagons represent HP 1 (Barbuy et al. 2016; tomato-filled pentagons show NGC 6558 (Barbuy et al. 2018c; magenta-filled pentagons represent M62 (Yong et al. 2014). The underlying coloured model corresponds to calculations for nucleosynthesis taking place in spinstars and in MRD supernovae (Cescutti et al. 2018 and references therein).

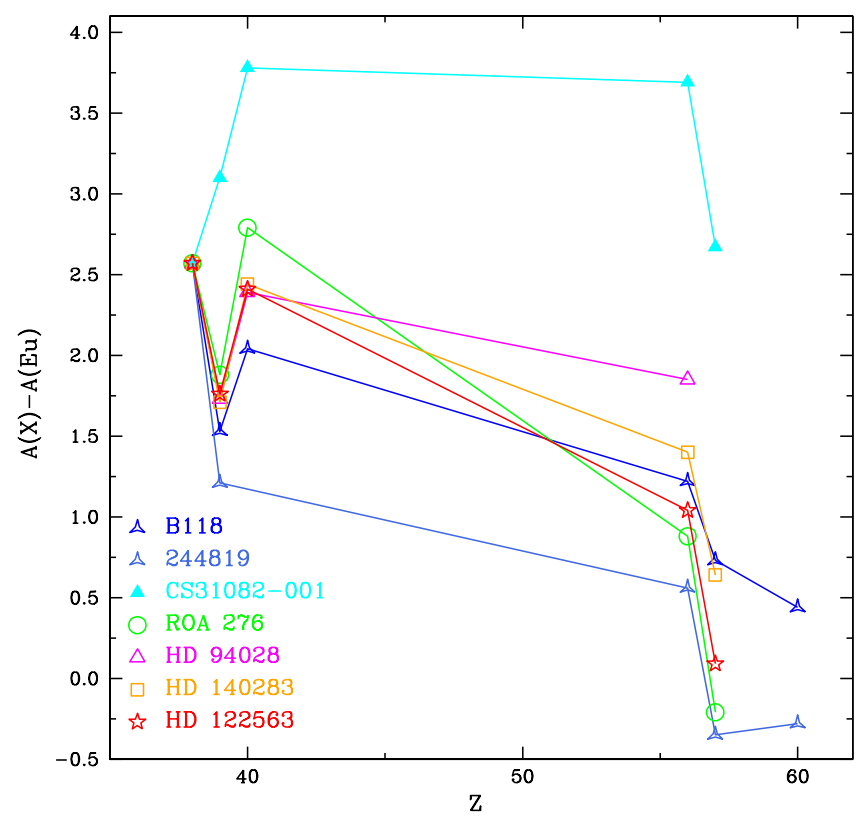

Fig. 17. $A(X)-A(E u)$ versus atomic number $Z$ for stars $B 118$ and 244819 compared with stars CS 31082-001, ROA 276, HD 94028, HD 140283, and HD 122563. All abundances are normalised to the Sr abundance of B118. Symbols are identified in the figure panel.

Finally, nucleosynthesis taking place in AGB stars and the iprocess might be alternative possibilities that should be further inspected.

Taking into account the different uncertainties at play, we confirm the conclusions from Barbuy et al. (2014) that the

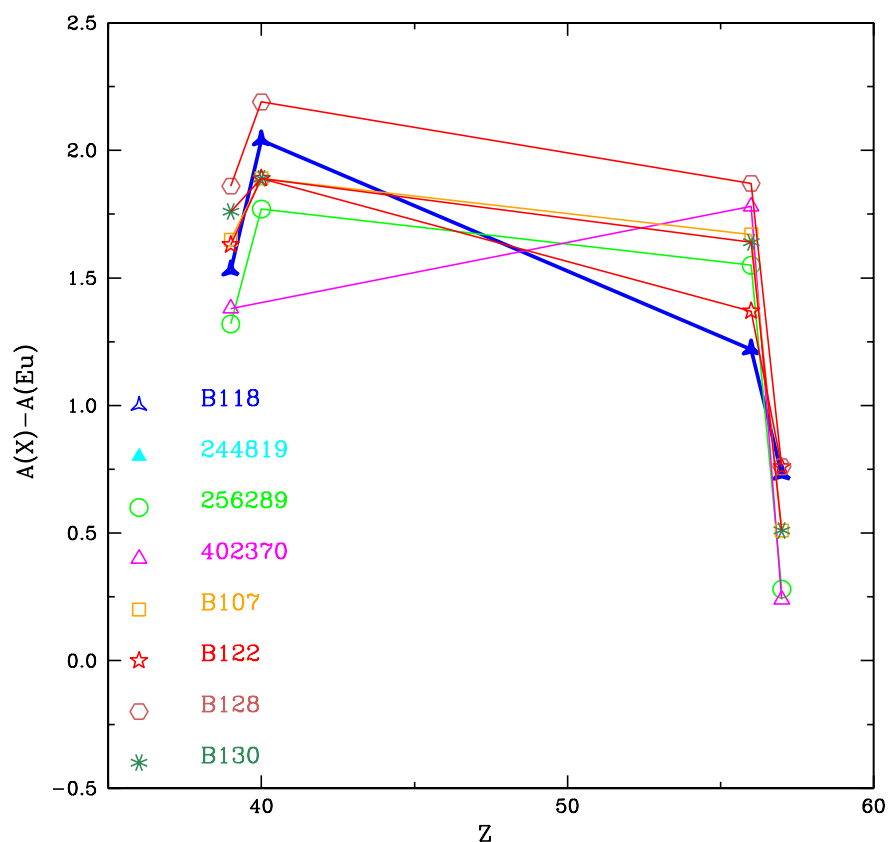

Fig. 18. $A(X)-A(E u)$ versus atomic number $Z$ for star $B 118$ compared with the other seven sample stars. The abundances are not normalised. Symbols are identified in the figure panel.

observed abundances are compatible with the $s$-process production in spinstars. However, we cannot rule out that the same enrichment signature could be produced by a combination of nucleosynthesis processes active in the early generations of stars.

Acknowledgements. B.B., E.C., L.M., S.O. acknowledge partial financial support from CAPES-Finance code 001, CNPq and FAPESP. SOS acknowledges the FAPESP PhD fellowship no. 2018/22044-3 and the DGAPA-PAPIIT grant IG100319. R.H. and M.P. acknowledges support from the IReNA AccelNet Network of Networks, supported by the National Science Foundation under Grant no. OISE-1927130 and from the World Premier International Research Centre Initiative (WPI Initiative), MEXT, Japan. C.C., R.H., M.P. and G.C. acknowledge support from the ChETEC COST Action (CA16117), supported by COST (European Cooperation in Science and Technology). MP thanks the support to NuGrid from STFC (through the University of Hull Consolidat ed Grant ST/R000840/1), and access to VIPER, the University of Hull High Performance Computing Facility. MP acknowledges the support from the Ledulet-2014 Program of the Hungarian Academy of Sciences (Hungary). SO acknowledges the Italian Ministero dell'Università e della Ricerca Scientifica e Tecnologica (MURST), Italy. This work has made use of data from the European Space Agency (ESA) mission Gaia (http://www. cosmos.esa.int/gaia), processed by the Gaia Data Processing and Analysis Consortium (DPAC, http://www. cosmos.esa. int/web/gaia/dpac/consortium). Funding for the DPAC has been provided by national institutions, in particular the institutions participating in the Gaia Multilateral Agreement.

\section{References}

Abate, C., Stancliffe, R. J., \& Liu, Z.-W. 2016, A\&A, 587, A50 Alonso, A., Arribas, S., \& Martínez-Roger, C. 1998, A\&AS, 131, 209

Alonso, A., Arribas, S., \& Martínez-Roger, C. 1999, A\&AS, 140, 261 (AAM99) Asplund, M., Grevesse, N., Sauval, A. J., \& Scott, P. 2009, ARA\&A, 47, 481 Arcones, A., \& Montes, F. 2011, ApJ, 731, 5

Baade, W. 1944, ApJ, 100, 137

Baade, W. 1946, PASP, 58, 249

Baba, J., \& Kawata, D. 2020, MNRAS, 492, 4500

Ballester, P., Modigliani, A., Boitquin, O., et al. 2000, Msngr, 101, 31B

Banerjee, P., Qian, Y.-Z., \& Heger, A. 2018, ApJ, 865, 120

Barbuy, B., Zoccali, M., Ortolani, S., et al. 2006, A\&A, 449, 349

Barbuy, B., Zoccali, M., Ortolani, S., et al. 2007, AJ, 134, 1613

Barbuy, B., Zoccali, M., Ortolani, S., et al. 2009, A\&A, 507, 405

Barbuy, B., Chiappini, C., Cantelli, E., et al. 2014, A\&A, 570, A76

Barbuy, B., Cantelli, E., Vemado, A., et al. 2016, A\&A, 591, A53 
Barbuy, B., Chiappini, C., Gerhard, O. 2018a, ARA\&A, 56, 223

Barbuy, B., Muniz, L., Ortolani, S., et al. 2018b, A\&A, 619, A178

Barbuy, B., Trevisan, J., \& de Almeida, A. 2018c, PASA, 35, 46

Baumgardt, H., Hilker, M., Sollima, A., \& Bellini, A. 2019, MNRAS, 482, 5138

Biehl, D. 1976, PhD Thesis, Kiel University

Bica, E., Ortolani, S., \& Barbuy, B. 2016, PASA, 33, 28

Bensby, T., Feltzing, S., Gould, A., et al. 2017, A\&A, 605, A89

Bessell, M. S. 1979, PASP, 91, 589

Bovy, J., Leung, H. W., Hunt, J. A. S., et al. 2019, MNRAS, 490, 4840

Buck, T., Ness, M., Macciò, A. V., Obreja, A., \& Dutton, A. A. 2018, ApJ, 861, 88

Cantelli, E. W. C. 2019, Master Thesis, USP

Carpenter, J. M. 2001, AJ, 121, 2851

Carretta, E., Bragaglia, A., Gratton, R. G., et al. 2009, A\&A, 505, 117

Casagrande, L., Ramírez, I., Meléndez, J., et al. 2010, A\&A, 512, A54

Cayrel, R. 1988, in The impact of very high S/N spectroscopy on Stellar Physics, eds. G. Cayrel de Strobel, \& M. Spite (Kluwer), Proc. IAU Symp., 132, 345

Cescutti, G., \& Chiappini, C. 2010, A\&A, 515, A102

Cescutti, G., \& Chiappini, C. 2014, A\&A, 565, A51

Cescutti, G., François, P., Matteucci, F., Cayrel, R., \& Spite, M. 2006, A\&A, 448,557

Cescutti, G., Chiappini, C., Hirschi, R., Meynet, G., \& Frischknecht, U. 2013, A\&A, 553, A51

Cescutti, G., Chiappini, C., \& Hirschi, R. 2018, IAU Symp. 334, 94

Chiappini, C. 2013, Astron. Nachr., 334, 595

Chiappini, C., Hirschi, R., Meynet, G., et al. 2006, A\&A, 449, L27

Chiappini, C., Frischknecht, U., Meynet, G., et al. 2011a, Nature, 472, 454 (C11a)

Chiappini, C., Frischknecht, U., Meynet, G., et al. 2011b, Nature, 474, 666 (C11b)

Choplin, A., Hirschi, R., Meynet, G., et al. 2018, A\&A, 618, A133

Clarson, O., Herwig, F., \& Pignatari, M. 2018, MNRAS, 474, L37

Cristallo, S., Straniero, O., Piersanti, L., \& Gorbrecht, D. 2015, ApJS, 219, 40

Debattista, V. P., Liddicott, D. J., Khachaturyants, T., \& Beraldo e Silva, L. 2020 , MNRAS, 498, 3334

Dekker, H., D’Odorico, S., Kaufer, A., Delabre, B., \& Kotzlowski, H. 2000, SPIE, 4008, 534D

Dotter, A., Chaboyer, B., Jevremovć, D., et al. 2008, ApJS, 178, 89

Edvardsson, B., Andersen, J., Gustafsson, B., et al. 1993, A\&A, 275, 101

Ernandes, H., Barbuy, B., Alves-Brito, A., et al. 2018, A\&A, 616, A18

Ernandes, H., Dias, B., Barbuy, B., et al. 2019, A\&A, 632, A103

Farouqi, K., Kratz, K.-L., \& Pfeiffer, B. 2009, PASA, 26, 194

Fernández-Trincado, J. G., Zamora, G., Souto, D., et al. 2019, A\&A, 627, A178

Fernández-Trincado, J. G., Minniti, D., Beers, T. C., et al. 2020, A\&A, 643, A145

Fragkoudi, F., Grand, R. J. J., Pakmor, R., et al. 2020, MNRAS, 494, 5936

Francois, P., Depagne, E., Hill, V., et al. 2003, A\&A, 403, 1105

Frischknecht, U., Hirschi, R., \& Thielemann, F.-K. 2012, A\&A, 538, L2

Frischknecht, U., Hirschi, R., Pignatari, M., et al. 2016, MNRAS, 456, 1803

Gaia Collaboration (Brown, A. G. A., et al.) 2018, A\&A, 616, A1

Gaia Collaboration (Brown, A. G. A., et al.) 2021, A\&A, 649, A1

Gnedin, O. Y., \& Ostriker, J. P. 1997, ApJ, 474, 223

Gratton, R. G., Carretta, E., \& Bragaglia, A. 2012, A\&ARv, 20, 50

Grevesse, N., \& Sauval, J. N. 1998, SSRev, 35, 161

Gustafsson, B., Edvardsson, B., Eriksson, K., et al. 2008, A\&A, 486, 951

Hampel, M., Stancliffe, R. J., Lugaro, M., \& Meyer, B. S. 2016, ApJ, 831, 171

Hanke, M., Koch, A., Prudil, Z., Grebel, E. K., \& Bastian, U. 2020, A\&A, 637, A98

Hansen, C., Montes, F., \& Arcones, A. 2014, ApJ, 797, 123

Hill, V., Plez, B., Cayrel, R., et al. 2002, A\&A, 387, 560

Hinkle, K., Wallace, L., Valenti, J., \& Harmer, D. 2000, Visible and Near Infrared Atlas of the Arcturus Spectrum 3727-9300 A, eds. K. Hinkle, L. Wallace, J. Valenti, \& D. Harmer (San Francisco: ASP)

Honda, S., Aoki, W., Ishimary, Y., \& Wanajo, S. 2007, ApJ, 666, 1189

Howes, L. M., Asplund, M., Keller, S. C., et al. 2016, MNRAS, 460, 884

Johnson, C. I., Rich, R. M., Kobayashi, C., \& Fulbright, J. P. 2012, ApJ, 749, 175

Kerber, L. O., Nardiello, D., Ortolani, S., et al. 2018, ApJ, 853, 15

Kunder, A., Pérez-Villegas, A., Rich, R. M., et al. 2020, AJ, 159, 270

Kurucz, R. 1993, CD-ROM 23

Lamb, M., Venn, K., Andersen, D., et al. 2017, MNRAS, 465, 3536
Limongi, M., \& Chieffi, A. 2018, ApJS, 237, 13

Madrid, J. P., Leigh, N. W. C., Hurley, J. R., \& Giersz, M. 2017, MNRAS, 470, 1729

Martin, W. C., Fuhr, J. R., Kelleher, D. E., et al. 2002, NIST Atomic Database (version 2.0), National Institute of Standards and Technology, Gaithersburg, MD http://physics.nist.gov/asd

Massari, D., Koppelman, H. H., \& Helmi, A. 2019, A\&A, 630, L4

McWilliam, A. 2016, PASA, 33, 40

McWilliam, A., Wallerstein, G., \& Mottini, M. 2013, ApJ, 778, 149

Meléndez, J., \& Barbuy, B. 2009, A\&A, 497, 611

Montes, F., Beers, T. C., Cowan, J., et al. 2007, ApJ, 671, 1685

Ness, M., Asplund, M., \& Casey, A. R. 2014, MNRAS, 445, 2994

Nishimura, N., Sawal, H., Taklwaki, T., Yamada, S., \& Thielemann, F.-K. 2017, ApJ, 836, L21

Ortolani, S., Bica, E., \& Barbuy, B. 2006, ApJ, 646, L115

Pasquini, L., Avila, G., Blecha, A., et al. 2002, The Messenger, 110, 1

Pérez-Villegas, A., Barbuy, B., Kerber, L., et al. 2020, MNRAS, 491, 3251

Peterson, R. C., Barbuy, B., \& Spite, M. 2020, A\&A, 638, A64

Pietrinferni, A., Cassisi, S., Salaris, M., \& Castelli, F. 2004, ApJ, 612, 167

Pietrinferni, A., Cassisi, S., Salaris, M., \& Castelli, F. 2006, ApJ, 642, 797

Pignatari, M., Gallino, M., Meynet, G., et al. 2008, ApJ, 687, L95

Piotto, G., King, I. R., Djorgovski, S. G., et al. 2002, A\&A, 391, 945

Piskunov, N., Kupka, F., Ryabchikova, T., Weiss, W., \& Jeffery, C. 1995, A\&AS, 112,525

Prantzos, N., Abia, C., Cristallo, S., Limongi, M., \& Chieffi, A. 2020, MNRAS, 491, 1832

Qian, Y.-Z., \& Wasserburg, G. J. 2008, ApJ, 687, 272

Queiroz, A. B. A., Anders, F., Chiappini, C., et al. 2020a, A\&A, 638, A76

Queiroz, A. B. A., Chiappini, C., Pérez-Villegas, A., et al. 2020b, ArXiv eprint [arXiv:2007.12915]

Rich, R. M., Ortolani, S., Bica, E., \& Barbuy, B. 1998, AJ, 116, 1295

Rich, R. M., Origlia, L., \& Valenti, E. 2012, ApJ, 746, 59

Riello, M., De Angeli, F., Evans, D. W., et al. 2021, A\&A, 649, A3

Rizzuti, F., Cescutti, G., Matteucci, F., et al. 2019, MNRAS, 489, 5244

Rizzuti, F., Cescutti, G., Matteucci, F., et al. 2021, MNRAS, 502, 2495

Roberts, L. F., Woosley, S. E., \& Hoffman, R. D. 2010, ApJ, 722, 954

Roederer, I. U., Cowan, J. J., Karakas, A. I., et al. 2010, ApJ, 723, 975

Roederer, I. U., Karakas, A. I., Pignatari, M., \& Herwig, F. 2016, ApJ, 821, 37

Rojas-Arriagada, A., Zasowski, G., Schultheis, M., et al. 2020, MNRAS, 499, 1037

Rossi, L., Ortolani, S., Bica, E., Barbuy, B., \& Bonfanti, A. 2015. MNRAS, 450, 3270

Rutten, R. J. 1978, SoPh, 56, 237

Ryabchikova T., Piskunov N., Kurucz R. L., et al. 2015, PhyS, 90, 054005

Saito, R., Hempel, M., Minnit, D., et al. 2012, A\&A, 537, A107

Savino, A., Koch, A., Prudil, Z., Kunder, A., \& Smolec, R. 2020, A\&A, 641, A96

Scott, P., Grevesse, N., Asplund, M., et al. 2015a, A\&A, 573, A26

Scott, P., Asplund, M., Grevesse, N., Bergemann, M. \& Sauval, A. J. 2015b, A\&A, 573, A27

Siqueira-Mello, C., Andrievsky, S., Barbuy, B., et al. 2015, A\&A, 584, A86

Siqueira-Mello, C., Chiappini, C., Barbuy, B., et al. 2016, A\&A, 593, A79

Skrutskie, M., Cutri, R. M., Stiening, R., et al. 2006, AJ, 131, 1163

Soto, M., Barbá, R., Gunthardt, G., et al. 2013, A\&A, 552, A101

Spite, F., Spite, M., Barbuy, B., et al. 2018, A\&A, 611, A30

Steffen, M., Prakapavicius, D., Caffau, E., et al. 2015, A\&A, 583, A57

Trevisan, M., Barbuy, B., Eriksson, K., et al. 2011, A\&A, 535, A42

Truran, J. W. 1981, A\&A, 97, 391

Udalski, A., Szymański, M., Kubiak, M., et al. 2002, AcA, 52, 217

van der Swaelmen, M., Barbuy, B., Hill, V., et al. 2016, A\&A, 586, A1

Willmer, C. N. A. 2018, ApJS, 236, 47

Winteler, C., Käppeli, R., Perego, A., et al. 2012, ApJ, 750, L22

Woosley, S. E., \& Weaver, T. A. 1995, ApJS, 101, 181

Yong, D., Alves-Brito, A., Da Costa, G., et al. 2014, MNRAS, 439, 2638

Yong, D., Norris, J. E., Da Costa, G. S., et al. 2017, ApJ, 837, 176

Zoccali, M., Lecureur, A., Hill, V., et al. 2008, A\&A, 486, 177 


\section{Appendix A: Candidate members observed with GIRAFFE}

Table A.1: OGLE and 2MASS numbers, coordinates, magnitudes including $J H K_{\mathrm{s}}$ from both $2 \mathrm{MASS}$ and VVV surveys, and final radial velocities for stars observed with GIRAFFE in 2012 and 2016.

\begin{tabular}{|c|c|c|c|c|c|c|c|c|c|c|c|c|c|c|}
\hline OGLE & 2MASS ID & $\alpha_{2000}$ & $\delta_{2000}$ & $V$ & $I$ & $J$ & $H$ & $K_{\mathrm{s}}$ & $J_{\mathrm{VVV}}$ & $H_{\mathrm{VVV}}$ & $K_{\mathrm{VVV}}$ & $v_{\mathrm{r}}^{\text {hel }}$ & $B_{\text {Johnson }}$ & Member \\
\hline \multicolumn{15}{|c|}{ GIRAFFE SAMPLE } \\
\hline \multicolumn{15}{|c|}{ GIRAFFE sample (2012) } \\
\hline 244853 & 18033424-3002109 & $18: 03: 34.13$ & $-30: 02: 11.1$ & 16.085 & 14.457 & 11.304 & 10.994 & 11.374 & - & - & - & -11.79 & 17.363 & 99.5 \\
\hline 402384 & 18034256-3001409 & $18: 03: 42.55$ & $-30: 01: 40.5$ & 16.055 & 14.388 & 13.259 & 12.506 & 12.336 & 13.0877 & 12.4530 & 12.2813 & -20.04 & 17.402 & 99.0 \\
\hline \multicolumn{15}{|c|}{ GIRAFFE sample (2016) } \\
\hline 244551 & 18033361-3002389 & 18:03:33.61 & $-30: 02: 38.9$ & 16.134 & 14.455 & 13.321 & 12.551 & 12.523 & 13.1628 & 12.5364 & 12.3582 & -8.63 & 17.486 & 97.8 \\
\hline 244555 & $18033467-3002305$ & 18:03:34.67 & $-30: 02: 32.2$ & 16.536 & 14.480 & 11.436 & 10.595 & 10.478 & - & - & - & -2.33 & 18.231 & 99.3 \\
\hline 244813 & - & 18:03:29.00 & $-30: 02: 28.3$ & 16.147 & 14.578 & - & - & - & 13.1904 & 12.5908 & 12.4422 & -10.80 & 17.365 & 43.7 \\
\hline 256298 & 18033214-3000350 & 18:03:32.13 & $-30: 00: 34.9$ & 16.038 & 14.430 & 13.133 & 12.306 & 12.173 & 13.1264 & 12.4863 & 12.2987 & -22.39 & 17.329 & 99.8 \\
\hline 402371 & 18033854-3002075 & 18:03:38.57 & $-30: 02: 07.3$ & 16.065 & 14.417 & 12.932 & 12.218 & 12.254 & 13.1894 & 12.6119 & 12.4397 & -17.52 & 17.394 & 99.6 \\
\hline 402508 & $18034025-3003178$ & 18:03:40.16 & $-30: 03: 18.1$ & 16.278 & 14.590 & 11.112 & 11.373 & 11.216 & 12.8682 & 12.0634 & 12.1385 & -4.32 & 17.593 & 91.9 \\
\hline
\end{tabular}

The radial velocities of GIRAFFE observations were measured by fitting the cross-correlation peak with the solar spectra available at the ESO portal ${ }^{6}$, and the heliocentric radial velocities were derived. The final heliocentric radial velocities of GIRAFFE spectra were determined by taking the average of the mean heliocentric velocity of each setup. This method showed a better agreement with their counterparts in the more reliable UVES spectra velocities. Signal to noise was measured in points of continua in each of the setups, resulting in a mean $S / N \sim 70$ in the HR11 setup and $S / N \sim 93$ in the HR12 setup.

We applied a selection of stars with radial velocities within $\pm 12 \mathrm{~km} \mathrm{~s}^{-1}$ of that of NGC 6522 to both the 2012 and the 2016 observations. On the selected sample, we combined the radial velocities with Gaia proper motions to derive membership probabilities. Table A.1 reports the selected stars, their identification, coordinates, magnitudes, and respective heliocentric radial velocities.

\section{Appendix B: Hyperfine structure of Ba II lines}

In Table B.1, we give the hyperfine structure constants for the BaII 5853.675 $\AA$ line, and in Table B.2 we report the list of lines sub-divided due to the hyperfine structure, thus completing the similar results for the 6141.713 and 6496.897 AA lines reported in Barbuy et al. (2014).

Table B.1: Atomic constants for BaII used to compute hyperfine structure.

\begin{tabular}{lccccccccccccc}
\hline \hline Species & $\lambda(\AA)$ & Lower level & $J$ & $A(\mathrm{mK})$ & $A(\mathrm{MHz})$ & $B(\mathrm{mK})$ & $B(\mathrm{MHz})$ & Upper level & $J$ & $A(\mathrm{mK})$ & $A(\mathrm{MHz})$ & $B(\mathrm{mK})$ & $B(\mathrm{MHz})$ \\
\hline${ }^{135} \mathrm{BaII}$ & 5853.668 & $5 \mathrm{~d}^{2} \mathrm{D}_{3 / 2}$ & $3 / 2$ & 3.56 & 106.7261 & 0 & 0 & $6 \mathrm{p}^{2} \mathrm{P}^{\circ}$ & $3 / 2$ & +3.47 & 104.028 & +2.2 & 65.9544 \\
${ }^{137} \mathrm{BaII}$ & 5853.668 & $5 \mathrm{~d}^{2} \mathrm{D}_{3 / 2}$ & $3 / 2$ & 3.97 & 119.0176 & 0 & 0 & $6 \mathrm{p}^{2} \mathrm{P}^{\circ}{ }^{3 / 2}$ & $3 / 2$ & +3.88 & 116.3195 & +3.25 & 97.4326 \\
${ }^{135} \mathrm{BaII}$ & 6141.713 & $5 \mathrm{~d}^{2} \mathrm{D}_{5 / 2}$ & $5 / 2$ & 1.49 & 44.6691 & 0 & 0 & $6 \mathrm{p}^{2} \mathrm{P}^{\circ} 3 / 2$ & $3 / 2$ & +3.47 & 104.028 & +2.2 & 65.9544 \\
${ }^{137} \mathrm{BaII}$ & 6141.713 & $5 \mathrm{~d}^{2} \mathrm{D}_{5 / 2}$ & $5 / 2$ & 1.66 & 49.7655 & 0 & 0 & $6 \mathrm{p}^{2} \mathrm{P}^{\circ} 3 / 2$ & $3 / 2$ & +3.88 & 116.3195 & +3.25 & 97.4326 \\
\hline
\end{tabular}

Notes. A constants are from Rutten (1978), B constants from Biehl (1976), and $B$ constants not available in the literature are assumed as null.

\footnotetext{
6 http://www.eso.org/observing/dfo/quality/GIRAFFE/

pipeline/solar.html
} 
Table B.2: Hyperfine structure for Ba II $5853.675 \AA$ A line.

\begin{tabular}{ccc}
\hline \hline \multicolumn{3}{c}{$5853.675 \AA$ ̊; $\chi=0.604321 \mathrm{eV}$} \\
\multicolumn{3}{c}{$\log g f$ (total) $=-1.10$} \\
$\lambda(\AA)$ & $\log g f$ & iso \\
5853.673 & -2.3441 & 135 \\
5853.674 & -2.3441 & 135 \\
5853.674 & -2.7421 & 135 \\
5853.673 & -2.1400 & 135 \\
5853.677 & -2.1400 & 135 \\
5853.676 & -2.0431 & 135 \\
5853.671 & -2.1980 & 135 \\
5853.679 & -2.1980 & 135 \\
5853.675 & -1.5959 & 135 \\
5853.673 & -2.3441 & 137 \\
5853.674 & -2.3441 & 137 \\
5853.674 & -2.7421 & 137 \\
5853.673 & -2.1400 & 137 \\
5853.677 & -2.1400 & 137 \\
5853.676 & -2.0431 & 137 \\
5853.670 & -2.1980 & 137 \\
5853.680 & -2.1980 & 137 \\
5853.674 & -1.5959 & 137 \\
5853.675 & -2.7562 & 134 \\
5853.675 & -2.2451 & 136 \\
5853.675 & -1.2845 & 138 \\
\hline
\end{tabular}




\section{Appendix C: Equivalent widths and atomic data}

Table C.1: Equivalent widths of Fe I and Fe II lines employed, their wavelengths, excitation potential (eV), oscillator strengths, and van der Waals broadening constant $\mathrm{C}_{6}$ adopted.

\begin{tabular}{|c|c|c|c|c|c|c|c|c|c|c|c|c|}
\hline \multirow{2}{*}{$\frac{\text { Specie }}{\text { Fe I }}$} & \multirow{2}{*}{$\begin{array}{l}\text { s } \lambda \\
5853.15\end{array}$} & \multirow{2}{*}{$\begin{array}{r}\chi_{\mathrm{ex}} \\
1.48\end{array}$} & \multicolumn{3}{|c|}{$\log g f$} & $\mathrm{C}_{6}$ & \multirow{2}{*}{$\begin{array}{r}234816 \\
43.4\end{array}$} & \multirow{2}{*}{$\begin{array}{r}244523 \\
13.9\end{array}$} & \multirow{2}{*}{$\begin{array}{r}244819 \\
-\end{array}$} & \multirow{2}{*}{$\begin{array}{r}256289 \\
-\end{array}$} & \multirow{2}{*}{$\begin{array}{r}402322 \\
9.6\end{array}$} & \multirow{2}{*}{402370} \\
\hline & & & - & 5.280 & - & $-5.130 .174 \mathrm{E}-31$ & & & & & & \\
\hline $\mathrm{Fe} I$ & 5856.08 & 4.29 & 1.640 & 1.328 & 8 & $-1.640 .423 E-30$ & 38.3 & - & 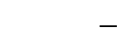 & - & 17.4 & . \\
\hline $\mathrm{Fe} I$ & 5858.78 & 22 & 2.260 & 2.260 & - & $-2.260 .376 E-30$ & 5.0 & - & - & & - & - \\
\hline $\mathrm{Fe} I$ & 5859.60 & 55 & 0.386 & -0.418 & - & $-0.600 .674 \mathrm{E}-30$ & 66.8 & 66.8 & - & - & 46.7 & - \\
\hline $\mathrm{Fe} I$ & 5861.11 & .28 & 2.450 & 2.450 & - & $-2.450 .415 \mathrm{E}-30$ & 7.0 & - & - & - & - & - \\
\hline e I & 5881.28 & 4.61 & -1.840 & -1.840 & - & $-1.840 .415 \mathrm{E}-30$ & 99. & - & - & . & 10.4 & - \\
\hline e I & 5902.48 & .59 & -1.810 & -1.810 & . & $-1.810 .708 \mathrm{E}-30$ & 6.6 & - & 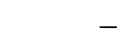 & - & 10.2 & - \\
\hline I & 5905.67 & & 0.730 & -0.730 & 77 & $730.623 E-30$ & 65.9 & 38.7 & 2.9 & 0.3 & 31.2 & - \\
\hline $\mathrm{Fe} I$ & 5916.25 & .45 & 2.994 & 2.994 & & $8 \mathrm{E}-31$ & 98.4 & 43.2 & 65.9 & 1.5 & 46.3 & 56.5 \\
\hline & 5927.79 & 65 & -1.090 & -1.090 & -1.07 & $-1.090 .786 \mathrm{E}-30$ & 27.6 & 42.0 & 9.7 & - & 16.1 & 12.4 \\
\hline $\mathrm{Fe} I$ & 5929.67 & 4.55 & -1.410 & -1.410 & -1.38 & $-1.380 .644 \mathrm{E}-30$ & 45.4 & 35.7 & 26.1 & - & 13.0 & 10.6 \\
\hline e I & 5930.18 & 65 & 0.230 & -0.230 & . & -0.230 .78 & 110.5 & - & - & 0.8 & 54.9 & 46.7 \\
\hline I & 5934.65 & & & -1.170 & & $E-30$ & 64.0 & 71.7 & 7.1 & - & 73.0 & 41.7 \\
\hline a & 5952.72 & 98 & 140 & -1.440 & & -1.390 .2 & 50.9 & 46.7 & 47.2 & 9.6 & 30.5 & 43.7 \\
\hline e I & 5956.69 & 86 & -4.605 & -4.605 & -4 & $-4.600 .948 E-32$ & 133.2 & 97.5 & 70.8 & - & 72.2 & 70.3 \\
\hline 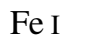 & 5975.35 & 84 & 0.822 & -1.292 & . & $-0.820 .300 \mathrm{E}-31$ & 37.1 & 42.0 & 26.3 & 9.5 & 44.3 & 36.3 \\
\hline & .68 & & & 0.485 & - & $806-2$ & 99. & 19.2 & & 5.3 & 88.0 & 3.1 \\
\hline e I & .06 & 99 & 54 & -0.432 & - & 20. & 99. & - & 0.0 & - & 33.9 & 37.2 \\
\hline II & 5991.38 & 3.15 & 57 & -3.540 & -3 & 540.7 & 18.4 & 18.4 & 19.6 & 29.0 & 27.2 & 8.9 \\
\hline Fe I & 6003.01 & 3.88 & -1.120 & -1.120 & - & -1.120 .21 & 91.2 & 81.1 & 62.5 & 63.8 & 75.3 & 44.2 \\
\hline$\pi$ & 05.54 & & & -3.602 & 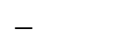 & 36103 & 18.4 & 9.7 & 8.4 & 4.3 & 20.8 & 14.9 \\
\hline I & 56 & 8 & & 0.982 & - & 31 & 89.3 & 1.7 & - & .3 & - & 55.1 \\
\hline 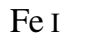 & .21 & 22 & 00 & -4.038 & -4 & -3.940 .3 & - & - & - & 17.8 & - & - \\
\hline $\mathrm{Fe} I$ & .17 & & 70 & -0.270 & - & 0.3 & 62.8 & 2.0 & 4.3 & - & 90.2 & - \\
\hline 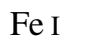 & .05 & & & 0.120 & - & -0.110 .6 & 99.9 & - & 1.9 & - & 68.7 & 77.8 \\
\hline & .06 & & & 1.089 & 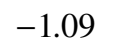 & -1.090 .2 & 65.6 & 4.1 & - & 2.3 & 2.8 & 39.0 \\
\hline e I & .01 & 3 & & -0.460 & - & 0.8 & 51.2 & 39.2 & 1.0 & 2.7 & 42.5 & 25.1 \\
\hline $\mathrm{F}$ & .49 & 61 & & -1.530 & -1 & -1.530 .4 & 145.0 & 116.9 & 116.0 & - & 31.3 & 112.4 \\
\hline $\mathrm{Fe} I$ & 78.50 & 4.79 & -0.481 & -0.323 & - & $-0.400 .951 \mathrm{E}-30$ & 60.1 & 47.5 & 50.5 & - & 60.2 & 53.0 \\
\hline & 9.00 & & & -1.120 & & 100. & 7.5 & & 0.0 & - & 28.2 & 12.7 \\
\hline I & 71 & 2 & & 3.573 & & 70. & 9.9 & 9.1 & 2.5 & - & 36.9 & 26.3 \\
\hline II & & & & -3.780 & & -3.790 & 7.9 & 9.1 & 9.1 & 5.1 & 12.0 & 5.2 \\
\hline $\mathrm{F}$ & 3.64 & & & -1.500 & & -1.470 .6 & 17.0 & & 3.7 & 14.8 & 14.2 & 10.2 \\
\hline $\mathrm{F}$ & 4.37 & 5 & & -1.940 & & -1.920 .7 & 11.7 & 99.0 & - & - & - & 2.3 \\
\hline $\mathrm{Fe} \mathrm{I}$ & 5.99 & 0 & 0 & -2.950 & -2 & $\begin{array}{ll}-2.95 & 0.2\end{array}$ & - & - & - & 5.8 & - & - \\
\hline $\mathrm{Fe} \mathrm{I}$ & & & & .403 & & 400 & 0.4 & 8.0 & 1.0 & 2.2 & - & 120.7 \\
\hline $\mathrm{Fe}$ II & 9.25 & 3.89 & -2.724 & -2.720 & -2 & -2.690 .9 & 5.6 & 4.3 & 7.6 & 0.0 & 18.9 & 12.1 \\
\hline Fe I & 1.62 & 2.18 & -3.299 & -3.299 & -3.30 & -3.300 .30 & 34.0 & 108.9 & 60.0 & 57.8 & 68.4 & 50.7 \\
\hline $\mathrm{Fe}$ & 7.73 & 8 & & -1.260 & -1 & $\begin{array}{ll}-1.25 & 0.2\end{array}$ & 71.1 & 24.5 & 59.8 & 43.4 & 59.8 & 43.2 \\
\hline $\mathrm{F}$ & & & & .970 & - & 50.6 & 2.1 & 9.0 & - & - & 11.5 & 5.0 \\
\hline $\mathrm{Fe} \mathrm{I}$ & 5.36 & & -1.550 & -1.474 & & -1.550 .2 & 40.7 & 2.4 & 3.7 & 24.1 & 26.3 & 23.8 \\
\hline $\mathrm{Fe} I$ & 73.33 & & -2.880 & -2.880 & & $-2.880 .882 \mathrm{E}-30$ & - & - & - & 75.8 & 80.1 & - \\
\hline Fo & & & & -2.586 & & -2.650 .5 & 2.6 & 94.8 & 60.0 & 2.1 & 81.3 & 49.0 \\
\hline $\mathrm{Fe} I$ & 7.99 & 94 & 4 & -1.720 & - & $\begin{array}{ll}-1.72 & 0.21\end{array}$ & 3.6 & 42.9 & 30.0 & - & 20.2 & 29.4 \\
\hline $\mathrm{Fe} \mathrm{I}$ & 00.31 & 2.61 & -2.437 & -2.437 & -1.67 & $-2.440 .507 \mathrm{E}-31$ & - & - & - & 75.5 & 134.1 & - \\
\hline $\mathrm{Fe} I$ & 13.43 & 2.22 & -2.660 & -2.482 & -2.48 & $-2.49 \quad 0.311 E-31$ & 111.6 & 89.2 & - & 92.0 & 99.4 & 87.7 \\
\hline Fe I & 19.28 & & & -2.433 & -2.43 & $-2.43 \quad 0.305 E-31$ & 83.0 & 143.6 & 98.4 & 98.3 & - & 97.3 \\
\hline & 20.78 & & -2.460 & -2.460 & - & $-2.460 .192 \mathrm{E}-30$ & 19.7 & - & - & - & 9.8 & 6.7 \\
\hline $\mathrm{Fe} I$ & 26.73 & 3.88 & -2.220 & -2.220 & - & $-2.20 \quad 0.191 \mathrm{E}-30$ & 36.6 & .9 & 7.3 & 2.1 & 21.1 & 9.2 \\
\hline $\mathrm{Fe} \mathrm{I}$ & 6229.23 & 2.84 & -2.970 & -2.805 & -2.805 & $-2.97 \quad 0.571 \mathrm{E}-31$ & 54.5 & 49.4 & 28.4 & 37.3 & 24.0 & 28.8 \\
\hline $\mathrm{Fe}$ & 240.65 & 2.22 & -3.380 & -3.233 & -3.17 & $-3.210 .309 E-31$ & 75.8 & 66.0 & 56.0 & 51.1 & 50.2 & 49.7 \\
\hline $\mathrm{F}$ & & 3.60 & & -0.733 & & $-0.88 \quad 0.1$ & 119.2 & 117.3 & 92.4 & 93.0 & - & 91.6 \\
\hline $\mathrm{Fe}$ II & 6247.56 & 3.89 & & -2.310 & & $-1.980 .881 \mathrm{E}-32$ & 22.4 & 51.1 & 31.8 & 47.8 & 50.1 & 22.3 \\
\hline $\mathrm{Fe} I$ & 6252.56 & 2.40 & -1.687 & -1.687 & -1.687 & $-1.690 .366 \mathrm{E}-31$ & 51.3 & 126.6 & 130.0 & 118.7 & 145.6 & 119.7 \\
\hline
\end{tabular}


Table C.1: continued.

\begin{tabular}{|c|c|c|c|c|c|c|c|c|c|c|c|}
\hline Species & $\lambda$ & $\chi_{e x}$ & & $\log g f$ & $\mathrm{C}_{6}$ & 234816 & 244523 & 244819 & 256289 & 402322 & 402370 \\
\hline $\mathrm{Fe} \mathrm{I}$ & 6254.25 & 2.28 & -2.480 & -2.443 & $-2.426-2.430 .326 \mathrm{E}-31$ & 166.0 & 129.7 & - & 113.1 & 113.5 & 107.9 \\
\hline Fe I & 6265.14 & 2.18 & -2.550 & -2.550 & $-2.55-2.530 .295 \mathrm{E}-31$ & 170.3 & 118.1 & 104.2 & - & 93.5 & 85.4 \\
\hline $\mathrm{Fe} \mathrm{I}$ & 6270.23 & 2.86 & -2.710 & -2.464 & $-2.61-2.610 .575 E-31$ & 76.1 & 70.1 & 57.5 & 57.5 & 61.4 & 54.8 \\
\hline $\mathrm{Fe} \mathrm{I}$ & 6271.28 & 3.33 & -2.950 & -2.703 & $-2.70-2.810 .945 E-31$ & 30.1 & 34.8 & 11.4 & - & 8.2 & 17.3 \\
\hline $\mathrm{Fe} \mathrm{I}$ & 6297.80 & 2.22 & -2.740 & -2.740 & $-2.74-2.740 .304 \mathrm{E}-31$ & 106.6 & 100.4 & 98.6 & 81.8 & 90.4 & 74.6 \\
\hline $\mathrm{Fe} \mathrm{I}$ & 6301.50 & 3.65 & -0.672 & -0.718 & $-0.72-0.600 .138 \mathrm{E}-30$ & 109.0 & 108.3 & 101.6 & 95.3 & 182.0 & 95.1 \\
\hline $\mathrm{Fe} \mathrm{I}$ & 6302.50 & 3.69 & -1.131 & -0.968 & $-0.910 .145 \mathrm{E}-30$ & 90.2 & 82.9 & - & 72.4 & - & - \\
\hline $\mathrm{Fe} \mathrm{I}$ & 6311.50 & 2.83 & -3.230 & -3.141 & $-3.14-3.220 .551 \mathrm{E}-31$ & 71.9 & 31.1 & 20.0 & 26.0 & 22.0 & 13.2 \\
\hline $\mathrm{Fe} \mathrm{I}$ & 6315.31 & 4.14 & -1.232 & -1.232 & $-1.232-1.230 .265 E-30$ & 44.4 & 27.0 & 39.2 & 42.2 & 44.6 & 35.5 \\
\hline $\mathrm{Fe} \mathrm{I}$ & 6315.81 & 4.08 & -1.710 & -1.710 & $-1.66-1.660 .243 \mathrm{E}-30$ & 38.8 & 40.8 & 25.0 & - & 31.6 & 15.4 \\
\hline $\mathrm{Fe} \mathrm{I}$ & 6322.69 & 2.59 & -2.426 & -2.426 & $-2.43-2.430 .485 \mathrm{E}-31$ & - & - & - & 74.6 & 96.5 & - \\
\hline $\mathrm{Fe} \mathrm{I}$ & 6335.33 & 2.20 & -2.230 & -2.177 & $-2.18-2.180 .295 \mathrm{E}-31$ & 135.3 & 96.1 & 120.0 & - & - & 105.0 \\
\hline $\mathrm{Fe} \mathrm{I}$ & 6336.83 & 3.69 & -1.050 & -0.856 & $-0.86-1.050 .143 \mathrm{E}-30$ & 94.9 & 107.9 & 98.3 & 83.9 & - & 79.7 \\
\hline $\mathrm{Fe} \mathrm{I}$ & 6344.15 & 2.43 & -2.923 & -2.923 & $-2.923-2.920 .366 \mathrm{E}-31$ & 81.1 & 81.8 & 62.0 & 61.9 & 68.5 & 61.8 \\
\hline $\mathrm{Fe} \mathrm{I}$ & 6355.03 & 2.85 & -2.420 & -2.350 & $-2.29-2.290 .549 \mathrm{E}-31$ & 90.9 & 93.2 & 90.0 & 68.7 & 83.0 & 48.2 \\
\hline Fe II & 6369.46 & 2.89 & -4.253 & -4.160 & $-4.29-4.110 .742 \mathrm{E}-32$ & $\ldots$ & - & - & 15.5 & 23.1 & - \\
\hline $\mathrm{Fe} \mathrm{I}$ & 6380.74 & 4.19 & -1.400 & -1.376 & $-1.38-1.380 .277 \mathrm{E}-30$ & 74.3 & 141.1 & - & 35.8 & 27.2 & 14.7 \\
\hline $\mathrm{Fe} \mathrm{I}$ & 6392.54 & 2.28 & -4.030 & -4.030 & $-4.030 .313 \mathrm{E}-31$ & 42.2 & 26.6 & - & - & 28.6 & 11.5 \\
\hline $\mathrm{Fe} \mathrm{I}$ & 6393.60 & 2.43 & -1.620 & -1.432 & $-1.576-1.580 .361 \mathrm{E}-31$ & 156.4 & 134.6 & - & 120.9 & 135.0 & 123.1 \\
\hline $\mathrm{Fe} \mathrm{I}$ & 6400.00 & 3.60 & -0.520 & -0.290 & $-0.290-0.290 .402 \mathrm{E}-30$ & - & - & - & 111.7 & - & - \\
\hline $\mathrm{Fe} \mathrm{I}$ & 6408.02 & 3.69 & -0.970 & -1.018 & $-1.02-1.000 .139 \mathrm{E}-30$ & 88.8 & 75.0 & - & 82.4 & 88.7 & - \\
\hline $\mathrm{Fe} \mathrm{I}$ & 6411.11 & 4.73 & -2.026 & -1.935 & $-2.210 .679 \mathrm{E}-30$ & - & - & - & - & - & - \\
\hline $\mathrm{Fe} \mathrm{I}$ & 6411.65 & 3.65 & -0.820 & -0.595 & $-0.72-0.720 .132 \mathrm{E}-30$ & 128.7 & 111.8 & 102.0 & 95.6 & 109.0 & 96.2 \\
\hline Fe II & 6416.92 & 3.89 & -2.740 & -2.650 & $-2.90-2.640 .930 \mathrm{E}-32$ & 28.3 & 36.4 & 20.5 & 26.1 & 44.0 & 19.8 \\
\hline $\mathrm{Fe} I$ & 6419.94 & 4.73 & -0.240 & -0.240 & $-0.27-0.250 .675 E-30$ & 54.0 & 60.2 & 54.2 & 52.9 & 66.6 & 45.7 \\
\hline $\mathrm{Fe} \mathrm{I}$ & 6421.35 & 2.28 & -2.027 & -2.027 & $-2.027-2.030 .310 \mathrm{E}-31$ & 122.5 & 121.9 & 120.0 & - & - & 108.1 \\
\hline $\mathrm{Fe} \mathrm{I}$ & 6430.85 & 2.18 & -2.006 & -2.006 & $-2.006-2.010 .281 \mathrm{E}-31$ & 137.9 & 129.9 & 131.0 & - & 130.0 & 113.3 \\
\hline Fe II & 6432.68 & 2.89 & -3.708 & -3.520 & $-3.50-3.570 .742 \mathrm{E}-32$ & 35.3 & 28.6 & 32.3 & 32.8 & 36.0 & 29.0 \\
\hline Fe II & 6456.38 & 3.90 & -2.075 & -2.100 & $-2.20-2.050 .930 \mathrm{E}-32$ & 41.4 & 33.9 & 27.2 & 51.9 & 54.7 & 24.7 \\
\hline $\mathrm{Fe} I$ & 6469.20 & 4.83 & -0.770 & -0.770 & $-0.81-0.810 .802 \mathrm{E}-30$ & 63.1 & 18.1 & 30.3 & - & 33.4 & 27.3 \\
\hline $\mathrm{Fe} \mathrm{I}$ & 6475.62 & 2.56 & -2.940 & -2.942 & $-2.94-2.940 .400 \mathrm{E}-31$ & 95.2 & 75.1 & 76.2 & 57.8 & 88.0 & 49.8 \\
\hline $\mathrm{Fe} \mathrm{I}$ & 6481.87 & 2.28 & -2.984 & -2.984 & $-2.98-2.980 .305 E-31$ & 98.9 & 50.2 & 76.2 & 67.3 & 78.8 & - \\
\hline $\mathrm{Fe} I$ & 6494.98 & 2.40 & -1.273 & -1.273 & $-1.273-1.270 .340 \mathrm{E}-31$ & 155.2 & 138.2 & 157.4 & 138.7 & 140.0 & 140.1 \\
\hline Fe II & 6516.08 & 2.89 & -3.450 & -3.320 & $-3.37-3.310 .742 \mathrm{E}-32$ & 52.2 & 55.3 & 29.3 & 55.2 & 48.9 & 35.1 \\
\hline $\mathrm{Fe} I$ & 6518.37 & 2.83 & -2.750 & -2.460 & $-2.30-2.300 .516 \mathrm{E}-31$ & 58.1 & 102.4 & - & 48.2 & 56.4 & - \\
\hline $\mathrm{Fe} \mathrm{I}$ & 6533.93 & 4.56 & -1.460 & -1.460 & $-1.430-1.430 .497 \mathrm{E}-30$ & 34.7 & 6.1 & 13.7 & - & - & 18.6 \\
\hline $\mathrm{Fe} \mathrm{I}$ & 6546.24 & 2.76 & -1.650 & -1.536 & $-1.54-1.540 .472 \mathrm{E}-31$ & 131.8 & 106.9 & 112.2 & 106.3 & - & 111.2 \\
\hline $\mathrm{Fe} \mathrm{I}$ & 6569.21 & 4.73 & -0.420 & -0.127 & $-0.45-0.450 .622 \mathrm{E}-30$ & 73.6 & 82.9 & 60.6 & 59.3 & - & 38.1 \\
\hline $\mathrm{Fe} \mathrm{I}$ & 6574.23 & 0.99 & - & -5.023 & $-5.004-5.000 .129 \mathrm{E}-31$ & - & - & - & 48.2 & - & - \\
\hline $\mathrm{Fe} \mathrm{I}$ & 6575.02 & 2.59 & -2.820 & -2.710 & $-2.710-2.710 .468 \mathrm{E}-31$ & - & - & - & 66.9 & 67.3 & - \\
\hline $\mathrm{Fe} \mathrm{I}$ & 6581.21 & 1.48 & -4.860 & -4.679 & $-4.68-4.850 .142 \mathrm{E}-31$ & 75.2 & 43.8 & 33.9 & - & 41.2 & 16.2 \\
\hline $\mathrm{Fe} \mathrm{I}$ & 6591.31 & 4.59 & -2.070 & -2.070 & $-2.000 .476 \mathrm{E}-30$ & 4.1 & - & - & - & - & 2.9 \\
\hline $\mathrm{Fe} \mathrm{I}$ & 6593.87 & 2.43 & -2.422 & -2.422 & $-2.42-2.420 .341 \mathrm{E}-31$ & 115.0 & 99.0 & - & 86.9 & 87.2 & 82.4 \\
\hline $\mathrm{Fe} \mathrm{I}$ & 6597.56 & 4.79 & -1.070 & -1.070 & $-1.05-1.050 .701 \mathrm{E}-30$ & 46.0 & 31.5 & - & 19.8 & - & 8.4 \\
\hline $\mathrm{Fe} \mathrm{I}$ & 6608.04 & 2.28 & -4.030 & -4.030 & $-4.030 .294 \mathrm{E}-31$ & 16.7 & 33.8 & 7.4 & 16.3 & 18.2 & 10.5 \\
\hline $\mathrm{Fe} \mathrm{I}$ & 6609.11 & 2.56 & -2.692 & -2.692 & $-2.69-2.690 .385 E-31$ & 94.6 & 29.4 & - & 69.3 & 54.9 & 67.1 \\
\hline $\mathrm{Fe} \mathrm{I}$ & 6627.54 & 4.55 & -1.680 & -1.680 & $-1.680 .437 \mathrm{E}-30$ & 13.3 & - & 10.7 & - & - & 9.4 \\
\hline $\mathrm{Fe} \mathrm{I}$ & 6678.00 & 2.69 & -1.470 & -1.418 & $-1.418-1.420 .428 \mathrm{E}-31$ & 160.0 & 200.0 & 124.0 & 120.9 & 130.0 & 117.4 \\
\hline $\mathrm{Fe} \mathrm{I}$ & 6699.14 & 4.59 & -2.190 & -2.101 & $-2.101-2.100 .452 \mathrm{E}-30$ & 5.2 & - & - & - & - & - \\
\hline $\mathrm{Fe} \mathrm{I}$ & 6705.10 & 4.61 & -1.470 & -1.382 & $-1.060 .467 \mathrm{E}-30$ & 38.1 & 30.1 & - & 26.4 & - & 3.3 \\
\hline $\mathrm{Fe} \mathrm{I}$ & 6726.67 & 4.61 & -0.952 & -1.094 & $-1.090 .447 \mathrm{E}-30$ & 32.5 & 36.4 & - & - & - & 18.7 \\
\hline $\mathrm{Fe} \mathrm{I}$ & 6739.52 & 1.56 & -4.950 & -4.794 & $-4.79-4.800 .147 \mathrm{E}-31$ & 42.7 & 18.5 & - & - & - & 8.9 \\
\hline
\end{tabular}

